
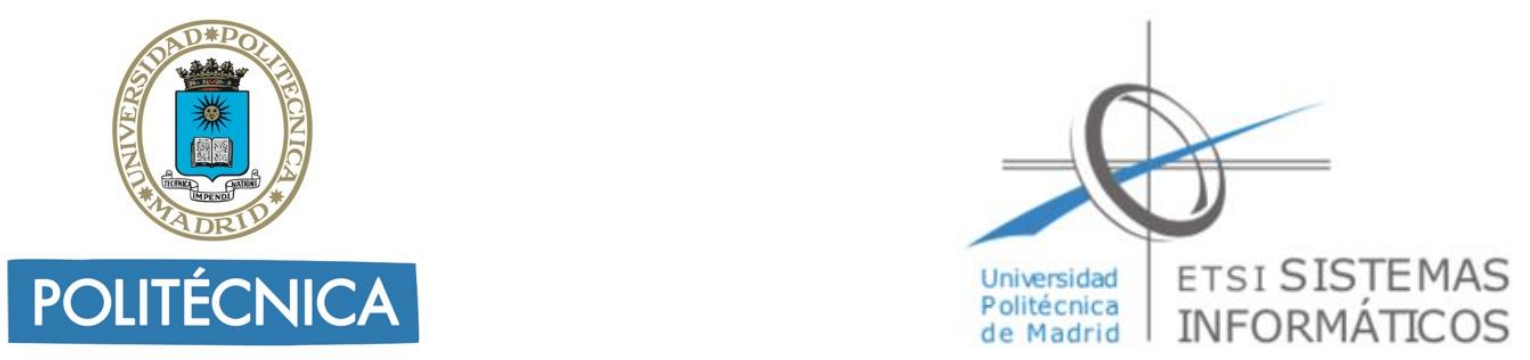

Universidad Politécnica de Madrid

\author{
ETS de Ingeniería de Sistemas \\ Informáticos
}

\title{
Una metodología sistemática para evaluar los modelos de predicción para la clasificación del estilo de conducción
}

\section{Tesis doctoral}

\author{
Iván Francisco Silva Feraud
}

Ingeniero en Ciencias Computacionales 


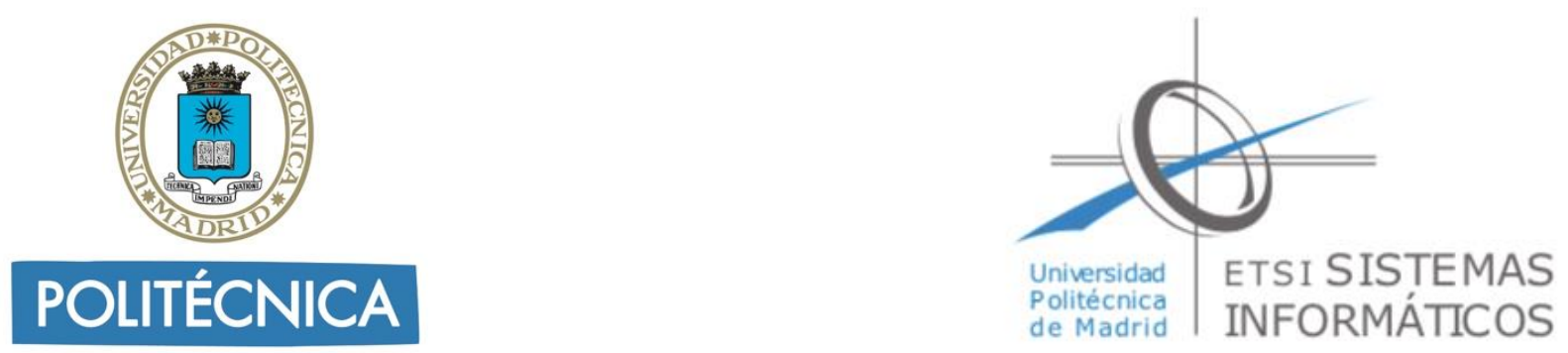

Departamento de Inteligencia Artificial

\author{
ETS de Ingeniería de Sistemas \\ Informáticos
}

\title{
Una metodología sistemática para evaluar los modelos de predicción para la clasificación del estilo de conducción
}

\section{Tesis doctoral}

Iván Francisco Silva Feraud

(Ingeniero en Ciencias Computacionales)

Director: José Eugenio Naranjo Hernández

(Doctor Ingeniero en Informática)

2020 

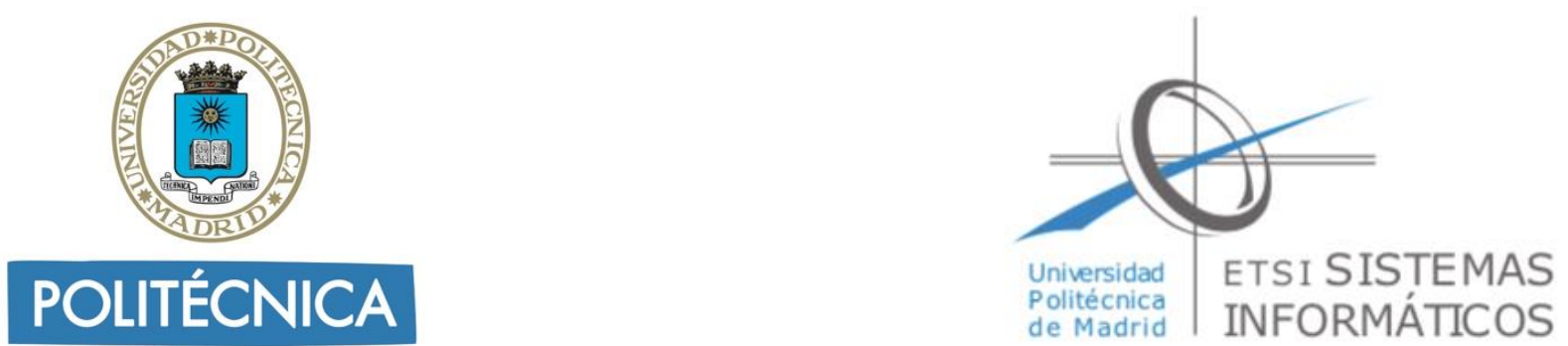

Tribunal nombrado por el Sr. Rector Magfco. De la Universidad Politécnica de Madrid, el día.... de. de $20 \ldots . .$.

Presidente:

Vocal:

Vocal:

Vocal:

Secretario:

Suplente:

Realizado el acto de defensa y lectura de la Tesis el día.... de. de $20 \ldots \ldots \ldots$ En la ETS de Ingeniería de Sistemas Informáticos.

Calificación

EL PRESIDENTE LOS VOCALES

EL SECRETARIO 


\section{Agradecimientos}

Empezare agradeciendo a Dios por darme la fuerza y sabiduría para avanzar en este largo camino del doctorado. A mi papá y mamá por siempre apoyarme en mi carrera e inculcarme que el estudio es importante en la vida. A mi tutor Eugenio por su paciencia, conocimiento y guía en estos años de estudio. Finalmente, a mi familia, a mis hijos Andrés y Cristina que con sus locuras alegraban mis días en los momentos más tenso del doctorado; y sobre todo a mi esposa y compañera Vanessa que sin su apoyo y dedicación no hubiera terminado mi doctorado. 


\section{Resumen}

La identificación del estilo de conducción mediante modelos de clasificación utilizando datos del vehículo, puede proporcionar información automatizada a los conductores sobre su comportamiento de conducción, especialmente si conducen de forma segura. Si bien en la bibliografía se han desarrollado varios modelos de clasificación para este propósito, no hay consenso sobre qué clasificador se desempeña mejor en la identificación del estilo de conducción. Por lo tanto, se necesita más investigación para evaluar los modelos de clasificación comparando métricas de desempeño. Este estudio tiene como objetivo el desarrollo de una serie de modelos para caracterizar la seguridad de la conducción de conductores, cuyo rendimiento será sistemáticamente analizado siguiendo metodologías bien establecidas para el aprendizaje automático y utilizando métricas de clasificación derivadas de la matriz de confusión y pruebas de significancia estadística. Los modelos seleccionados han sido desarrollados utilizando las siguientes técnicas de inteligencia computacional: lógica difusa, ANN, SVM, RF y kNN. Para entrenar y probar los cinco modelos de clasificación, se recopilo eventos agresivos e infracciones de tránsito, a través de dispositivos electrónicos en un entorno real. Los resultados experimentales muestran que SVM superó a los otros cuatro modelos con una exactitud de 0.96 y cuatro de los cinco modelos predijeron los estilos de conducción de forma similar. 


\section{Abstract}

Identifying driving styles using classification models with in-vehicle data can provide automated feedback to drivers on their driving behavior, in particularly if they are driving safely. While several classification models have been developed in the literature for this purpose, there is no consensus regarding which classifier performs better at identifying driving styles. Therefore, more research is needed to evaluate classification models by comparing performance metrics. This study aims to develop a series of models to characterize the driving safety of human drivers whose performance has been systematically analyzed following wellestablished methodologies for machine learning and using classification metrics derived from the confusion matrix and statistical significance tests. The selected models have been developed using the following computational intelligence techniques: fuzzy logic, ANN, SVM, RF and kNN. In order to train and test the five classification models, we collected aggressive events and traffic violations, through OBD2 and GPS devices in a naturalistic setting. Experimental results show that SVM outperformed the other four models with an accuracy of 0.96 and four of the five models predicted the driving styles in a similar way. 


\section{Índice General}

Agradecimientos __ iii

Resumen___ iv

Abstract___ v

Índice General___ vi vi

Índice de Figuras___ ix

Índice de Tablas __ X

Trabajos Realizados ___ $\mathrm{xi}$

Capítulo 1: Introducción___ 1

$1.1 \quad$ Motivación y contexto___ 1

1.2 Objetivos _ـ 3

1.2.1 Objetivo general___ 3

1.2.2 Objetivos específicos___ 3

1.3 Estructura de la tesis __ 4

Capítulo 2: $\quad$ Proceso de Implementación de Modelos Computacionales __ 7

2.1 Introducción _ 7

2.2 Recolección de datos___ 9

2.3 Generación de variables de entrada __ 10

2.4 Construcción del modelo computacional ___ 12

2.4.1 Configuración del modelo computacional __ 12

2.4.2 Entrenamiento del modelo computacional ___ 15

$2.5 \quad$ Evaluación del modelo computacional ___ 22

2.5.1 Métricas de la matriz de confusión___ 22

2.5.2 Pruebas estadísticas __ 25

2.6 Resumen 26

Capítulo 3: Estado del Arte del Estilo de Conducción __ 27

3.1 ¿Qué es el estilo de conducción? 27

3.2 Estilo de conducción agresivo __ 29

vi Una metodología sistemática para evaluar los modelos de predicción para la clasificación del estilo de conducción 
3.3 Métodos para determinar el estilo de conducción 30

3.3.1 Cuestionarios de autoevaluación___ 30

3.3.2 Análisis de datos del vehículo ___ 32

3.4 Clasificación automática del estilo de conducción ___ 32

3.4.1 Recolección de datos __ 33

3.4.2 Variables de entrada __ 33

3.4.3 Etiquetas o clases _ 35

3.4.4 Modelos computacionales ___ 37

3.5 Limitaciones y desafíos ___ 42

3.6 Resumen _ 44

Capítulo 4: Clasificación automática de estilos de conducción ___ 45

4.1 Metodología para la clasificación automática de estilos de conducción __ 45

4.2 Experimento ilustrativo de la metodología propuesta___ 47

4.2.1 Recolección de datos __ 47

4.2.2 Variables de entrada

4.2.3 Construcción de modelos computacionales___ 57

4.2.4 Evaluación de modelos ___ 64

4.3 Resumen 67

Capítulo 5: Discusión___ 69

$5.1 \quad$ Evaluación de desempeño y la prueba estadística ___ 69

5.2 Una metodología para la clasificación del estilo de conducción __ 73

Capítulo 6: Conclusiones __ 78

6.1 Limitaciones __ 79

6.2 Trabajos futuros

Bibliografía __ 81

Apéndice _ 90

Código del modelo borroso en MATLAB__ 90

Código del modelo de redes neuronales en RStudio ___ 92

Código del modelo de máquinas de soporte vectorial en RStudio ___ 92

Una metodología sistemática para evaluar los modelos de predicción para la clasificación del estilo de 


\section{Índice de Figuras}

Figura 2.1 Proceso iterativo del modelo computacional .......................................................................

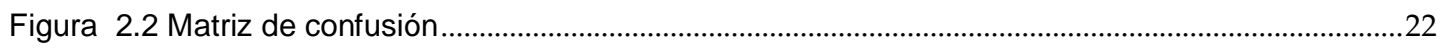

Figura 4.1 Metodología propuesta para la clasificación automática de estilos de conducción. ...............46

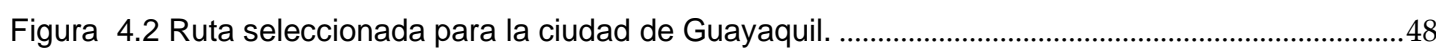

Figura 4.3 Ruta seleccionada para la ciudad de Madrid. ................................................................................49

Figura 4.4 Arquitectura del modelo para estimar el comportamiento de conducción segura a través de las infracciones de tránsito.

Figura 4.5 Descripción de las variables de entrada con sus etiquetas lingüísticas y funciones de pertenencia.

Figura 4.6 Correlación entre los puntos de penalización y la estimación del comportamiento de conducción segura.

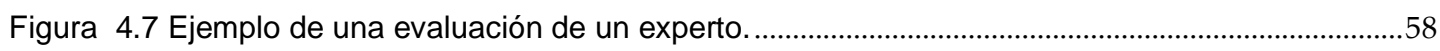

Figura 4.8: Variables de entrada con sus respectivas funciones de pertenencia. ...................................61

Figura 5.1 Resultados de los modelos computaciones. ..........................................................................69

Figura 5.2 Resultado por categoría por modelo computacional ................................................................72 


\section{Índice de Tablas}

Tabla 2.1 Síntesis de las etapas de implementación de un modelo computacional.....................................8

Tabla 2.2 Rango de valores para interpretar el indica kappa..................................................................2

Tabla 2.3 Rango de valores para interpretar la métrica AUC ....................................................................2

Tabla 3.1 Variables de entradas extraídas o derivadas del vehículo. ............................................................34

Tabla 3.2 Síntesis de las categorías de estilos de conducción utilizados en trabajos previos...................36

Tabla 3.3 Modelos computacionales más utilizados para identificar el estilo de conducción, junto con su configuración y métricas para medir su rendimiento. ..................................................................38

Tabla 4.1 Umbrales definidos para eventos de aceleración brusca y de frenado brusco dependiendo del nivel de flujo de tráfico.

Tabla 4.2 Reglas definidas para las variables de entrada. . .55

Tabla 4.3 Reglas definidas a partir de las variables de entrada. . .62

Tabla 4.4 Valores de búsqueda cuadricula para redes neuronales artificiales (ANN).

Tabla 4.5 Valores de búsqueda cuadricula para la máquina de soporte vectorial (SVM).

Tabla 4.6 Métricas de evaluación (exactitud, puntuación F1, Área bajo la curva (AUC), Kappa) para cada modelo. $A N N$ = redes neuronales artificiales; $S V M=$ Máquinas de soporte vectorial; $\mathrm{RF}=$ Bosques aleatorios; $\mathrm{kNN}=\mathrm{k}$-vecino más cercano.

Tabla 4.7 Puntaje F1 y AUC por cada estilo de conducción

Tabla 4.8 Valores de $p$ correspondientes a la prueba de significancia de Wilcoxon 


\section{Trabajos Realizados}

Las siguientes publicaciones revisadas por pares, fueron producidas durante del doctorado contribuyendo a esta tesis.

\section{Artículos de Revistas}

- Silva, I., \& Eugenio Naranjo, J. (2020). A Systematic Methodology to Evaluate Prediction Models for Driving Style Classification. Sensors, $20(6), 1692$.

\section{Artículos de Conferencias}

- Feraud, I. S., Lara, M. M., \& Naranjo, J. E. (2017, October). A fuzzy logic model to estimate safe driving behavior based on traffic violation. In 2017 IEEE Second Ecuador Technical Chapters Meeting (ETCM) (pp. 1-6). IEEE.

- Feraud, I. S., \& Naranjo, J. E. (2019, January). Are you a Good Driver? A Data-driven Approach to Estimate Driving Style. In Proceedings of the 11th International Conference on Computer Modeling and Simulation (pp. 3-7). 



\section{Capítulo 1: Introducción}

Esta tesis investiga el diseño, validación y evaluación de modelos computacionales para clasificar el estilo de conducción de automóviles, utilizando datos obtenidos de situaciones reales de conducción. Este capítulo está estructurado de la siguiente manera: la Sección 1.1 presenta el contexto y la motivación de esta investigación. La Sección 1.2 describe los objetivos de la tesis. Finalmente, la Sección 1.3 proporciona un resumen de la estructura de la tesis con una descripción general de los capítulos restantes.

\subsection{Motivación y contexto}

El estilo de conducción es un factor importante para garantizar la seguridad vial y se define como la forma en que los conductores manejan, así como sus comportamientos y hábitos asociados, desarrollados a lo largo de los años [1]. Petridou y Moustaki sugieren que el estilo de conducción agresivo es una de las causas más importantes de accidentes de tránsito [2] provocando muertes, lesiones graves y pérdidas materiales. La Organización Mundial de la Salud (OMS) ha informado que 1.2 millones de personas mueren cada año por accidentes de tránsito, y 20 millones quedan gravemente heridas [3]. Además, la Asociación Americana del Automóvil (AAA) también ha indicado que el estilo de conducción agresivo es la tercera causa de accidentes en los Estados Unidos [4].

La identificación del estilo de conducción puede brindar retroalimentación efectiva y personalizada de acuerdo al desempeño del conductor, ofreciendo varios beneficios, como la reducción del consumo de energía [5] y, la disminución de las probabilidades de accidentes de tránsito [6]. Expertos e investigadores en esta área, han empezado a desarrollar aplicaciones para identificar el estilo de conducción [7] [8] [9]. Por ejemplo, las compañías de seguros están desarrollando sistemas automáticos para determinar el estilo de conducción de sus clientes con el fin de ofrecer pólizas a precios ajustados [8]. Además, en el área de los Sistemas Avanzados de Asistencia al Conductor (SAAC), 
investigadores han mostrado interés en el desarrollo de herramientas inteligentes para mejorar la experiencia al conducir y la seguridad del mismo a través de dispositivos electrónicos [9].

Estudios recientes han reportado diferentes metodologías de modelos computacionales para clasificar los estilos de conducción recopilando datos a través de sensores acoplados al vehículo o de sensores externos, como, por ejemplo, utilizando sensores de los teléfonos inteligentes [7, 10-12]. Estas investigaciones proponen diferentes i) variables de entrada que se pueden extraer y transformar de los datos recopilados (p. ej. aceleración, desaceleración, frenado) $[13,14]$; ii) modelos computacionales para clasificar los estilos de conducción [10,15]; iii) etiquetas del estilo de conducción (p. ej. calmada, normal, agresivo) [16, 17]; y iv) métricas que evalúan los modelos de clasificación [18, 19]. Aunque estos estudios han propuesto una clasificación empírica de los estilos de conducción, pocos estudios han evaluado sistemáticamente cada uno de los componentes necesarios para identificar de mejor manera el estilo de conducción.

Por otro lado, diferentes modelos de inteligencia artificial, como por ejemplo, bosques aleatorios (Random Forest (RF), por sus siglas en inglés), las redes neuronales artificiales (Artificial Neural Networks (ANN), por sus siglas en inglés), K-vecinos más cercanos (K-Nearest-Neighbor (kNN), por sus siglas en inglés), y las máquinas de soporte vectorial (Support Vector Machines (SVM), por sus siglas en inglés), han sido implementados para determinar el estilo de conducción en diferentes contextos de conducción segura, como por ejemplo, para estimar los eventos del conductor [10], las maniobras de estilo de conducción [19], y la agresividad del conductor [18].

Sin embargo, pocos trabajos de investigación han realizado una evaluación formal y sistemática de estos modelos computacionales [7, 11]. Una investigación realizada por Meiring y Myburgh [11] sugiere que la lógica borrosa, debido a que puede utilizarse como una estrategia de clasificación y SVM pueden considerarse los mejores modelos computacionales para identificar los estilos de conducción. Además, en una revisión de literatura de 30 artículos, Martinez et al. [7] indicó que la lógica borrosa, kNN y ANN son los clasificadores más utilizados para identificar los estilos de conducción. Hasta el momento, no 
se ha establecido una metodología sistemática para evaluar diferentes modelos computacionales que permitan clasificar de manera efectiva el estilo de conducción en las mismas condiciones (p. ej. métricas de rendimiento, variables de entrada, posibles salidas), puesto que la mayoría de los trabajos previos no reportan la implementación o evaluación completa de dichos modelos computacionales.

Por los motivos antes descritos, esta tesis propone la siguiente pregunta de investigación: ¿Cómo se puede proveer de una metodología sistematizada que permita implementar y evaluar el estilo de conducción utilizando modelos computacionales? Específicamente, este trabajo se centra en el uso de datos de un vehículo, los cuales capturan eventos de conducción de forma natural, siendo un método no intrusivo para el conductor. Adicionalmente, este trabajo examina la forma de automáticamente detectar infracciones de tránsito, aprovechando el uso de los datos del vehículo.

A continuación, este capítulo presenta el objetivo general y los objetivos específicos que se abordaran para esta problemática.

\subsection{Objetivos}

\subsubsection{Objetivo general}

El objetivo principal de esta tesis es el de proponer una metodología que permita realizar una evaluación sistemática de diferentes modelos computacionales, bajo las mismas condiciones, para identificar el estilo de conducción de automóviles desde una perspectiva de conducción segura.

\subsubsection{Objetivos específicos}

Los siguientes objetivos específicos fueron establecidos para cumplir con el objetivo general:

1. Determinar los componentes de la metodología que permita la implementación y evaluación sistémica de estilos de conducción, con base en la literatura del estilo de conducción y guías prácticas de implementación y evaluación de modelos computacionales. 
2. Diseñar e implementar un modelo computacional que permita estimar las infracciones de tránsito aprovechando los datos capturados del vehículo.

3. Implementar varios modelos computacionales que permitan estimar el estilo de conducción en el contexto de seguridad vial.

4. Evaluar y seleccionar el o los mejores modelos computacionales en el ámbito del estilo de conducción.

\subsection{Estructura de la tesis}

Para poder abordar los objetivos planteados, esta tesis se ha divido en 6 capítulos:

- Capítulo 2: Introducción. En este capítulo se revisa la teoría y las mejores prácticas del proceso de implementación de modelos computacionales, basados en los modelos de aprendizaje de máquinas. La definición de este proceso ayudará a establecer la metodología propuesta.

- Capítulo 3: Estado del arte del estilo de conducción. Este capítulo describe los conceptos sobre del estilo de conducción, los fundamentos, la definición y los enfoques para identificar el estilo de conducción. Este capítulo es clave, puesto que también presenta las limitaciones y desafíos encontrados en la literatura, lo que permite definir varios pasos en la metodología que se propone en el siguiente capítulo.

- Capítulo 4: Clasificación automática del estilo de conducción. En primera instancia, este capítulo formaliza la metodología para evaluar sistematizadamente los diferentes modelos computacionales que permitan clasificar los estilos de conducción. Esta metodología, fundamentada en literatura previa, en las mejores prácticas para proyectos de aprendizaje automático, y en las limitaciones encontradas en los trabajos previos, consta de cuatro pasos: 1) recolección de datos; 2) variables de entrada; 3) construcción de modelos y 4) evaluación de modelos computacionales. En segunda instancia, este capítulo describe el 
diseño e implementación de un modelo que permite estimar las infracciones de tránsito utilizando los datos recopilados del vehículo. Finalmente, este capítulo explica el experimento que ilustra la aplicación de la metodología propuesta.

- Capítulo 5: Discusión. En este capítulo se discuten los resultados obtenidos de las métricas de desempeño y la validación estadística de los modelos computacionales implementados en el experimento, así como los aspectos relevantes que se abordaron en la metodología propuesta.

- Capítulo 6: Conclusiones. En este capítulo se exponen las conclusiones del trabajo de investigación, incluyendo las contribuciones, limitaciones de este y los posibles trabajos futuros. 



\section{Capítulo 2: Proceso de Implementación de Modelos Computacionales}

Este capítulo revisa los fundamentos teóricos y prácticos necesarios para el desarrollo e implementación de modelos computacionales de forma general. La revisión de esta teoría es importante debido a que son las bases para implementar cualquier modelo computacional de aprendizaje automático. Este capítulo está divido en dos partes: la primera parte describe el proceso general de implementación de modelos computacionales. En esta revisión se definen seis etapas, las cuales incluyen desde la definición del problema hasta la publicación y monitoreo del modelo computacional. Esta parte también describe las técnicas para configurar y evaluar los modelos computacionales. Adicionalmente, la teoría se entrelaza con las buenas prácticas de implementación, para poder abordar diferentes tópicos relacionados a las propias características de los datos y soluciones reales, como lo son: sobreajuste, datos balanceados y no balanceados, entre otros. La segunda parte de este capítulo detalla los fundamentos teóricos de los modelos computacionales más relevantes en la literatura, como lo son: lógica difusa, redes neuronales, máquinas de soporte vectorial, bosques aleatorios, entre otros. Este capítulo cierra con un resumen sobre los aspectos más importantes a considerar cuando se implementa un modelo computacional.

\subsection{Introducción}

La implementación de un modelo computacional es un proceso iterativo que comienza con la definición del problema que se desea solucionar hasta poner el modelo en producción [20-22]. Tal como se puede observar en la Tabla 2.1. Lakshmanan, Smith y Harrington proponen una serie de etapas que comprenden el proceso de implementación de modelos computacionales. 
Tabla 2.1 Síntesis de las etapas de implementación de un modelo computacional

\begin{tabular}{|l|c|c|c|}
\hline Etapas & Lakshmanan[21] & Smith[20] & Harrington[22] \\
\hline Definición de problema & $\mathrm{X}$ & & \\
\hline Recolección de datos & $\mathrm{X}$ & $\mathrm{X}$ & $\mathrm{X}$ \\
\hline Selección de algoritmos & $\mathrm{X}$ & & \\
\hline Generación de características & $\mathrm{X}$ & $\mathrm{X}$ & $\mathrm{X}$ \\
\hline Construcción del modelo & $\mathrm{X}$ & $\mathrm{X}$ & $\mathrm{X}$ \\
\hline Evaluación del modelo & $\mathrm{X}$ & $\mathrm{X}$ & $\mathrm{X}$ \\
\hline Presentación de resultados & $\mathrm{X}$ & & \\
\hline $\begin{array}{l}\text { Plan para despliegue del } \\
\text { modelo }\end{array}$ & $\mathrm{X}$ & & \\
\hline Publicación el modelo & $\mathrm{X}$ & $\mathrm{X}$ & $\mathrm{X}$ \\
\hline Monitoreo del modelo & $\mathrm{X}$ & $\mathrm{X}$ & \\
\hline
\end{tabular}

Tomando en consideración estas tres propuestas y sus similitudes, este trabajo deriva un proceso iterativo de seis etapas para implementar un modelo computacional. La Figura 2.1, muestra el proceso iterativo derivado, el cual consta de las siguientes etapas: 1) recolección de datos, 2) generación de características, 3) construcción del modelo, 4) evaluación del modelo, 5) publicación del modelo y 6) monitoreo del modelo. Las dos últimas etapas no serán descritas en este trabajo debido a que no están en el alcance de investigación. A continuación, se presentan los conceptos y técnicas para desarrollar cada una de estas etapas, orientado a la identificación de estilos de conducción.

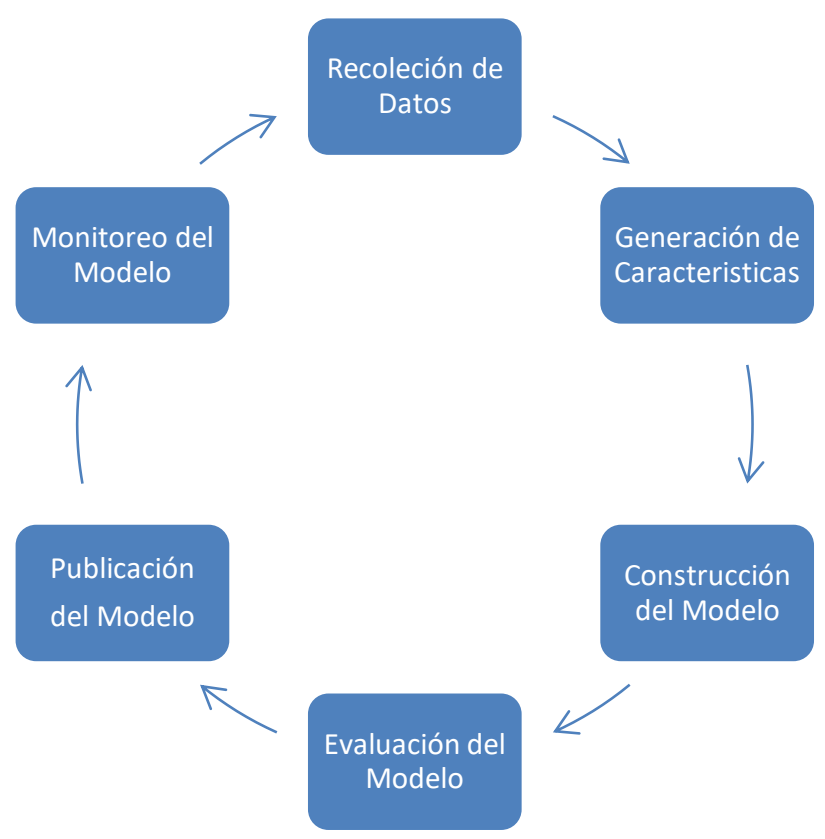

Figura 2.1 Proceso iterativo del modelo computacional 


\subsection{Recolección de datos}

La recopilación de datos es un proceso para colectar observaciones o mediciones de una manera sistemática, y obtener conocimientos de un tema en particular [23]. Antes de recopilar los datos se debe definir el objetivo de la investigación, el tipo de datos y los instrumentos que se utilizarán.

Los datos pueden ser organizados en dos grandes tipos: datos cualitativos y cuantitativos. Los datos cualitativos en su mayoría no son numéricos y por lo general son de naturaleza descriptiva o nominal. Esto quiere decir que los datos no son cuantificables y están en forma de palabras o texto. Los estudios cualitativos tienen como objetivo garantizar una mayor comprensión del problema. Los instrumentos de recopilación de datos suelen ser entrevistas, grupos focales, casos de estudio, entre otros [24].

Los datos cuantitativos se presentan en forma de números, cantidades o valores; y requieren un cálculo matemático para deducirlos. Este tipo de datos trata características que puedan ser medibles y se puedan expresar en números como por ejemplo temperatura, tamaño, duración, longitud, etc. Con los datos cuantitativos se elaboran análisis y se puede establecer conclusiones con mayor confianza que los datos cualitativos. Los instrumentos más utilizados son cuestionarios de preguntas cerradas, sistemas de información, experimentos, entre otros [24].

La elección entre recolectar datos cualitativos o cuantitativos depende del área de investigación y de la naturaleza de los objetivos. Tal como se mencionó en la sección 1.1, la motivación de esta investigación es identificar el estilo de conducción realizando pruebas reales de manejo, por lo tanto, este trabajo utilizará datos cuantitativos obtenidos de experimentos. Se puede definir que un experimento es la observación de un sistema o proceso para la compresión y aprendizaje de un tema en particular [25].

El experimento se realizará a través de sensores, debido a que se pueden recolectar datos de forma automática. Además, los sensores son más exactos cuando realizan una medición y esto hace que las conclusiones sean más robustas. Se debe de tomar en cuenta que los sensores por lo general necesitan calibrarse, debido a que algunos cuentan con un margen de error o pueden estar 
influenciados por las condiciones ambientales, como lo menciona Arampatzis et al. en su investigación [26]. También se debe tomar en consideración que los datos obtenidos por los sensores por lo general son "datos en bruto", a los cuales se le debe aplicar un algoritmo de pre-procesamiento para que puedan ser utilizados por los modelos computacionales [27].

\subsection{Generación de variables de entrada}

Una variable es una característica medible que se desea analizar, la cual actúa como entrada en el modelo computacional. Lo que se espera en esta etapa es crear un conjunto de datos para entrenar y validar el modelo. La generación de variables de entradas es una etapa crucial debido a que tiene un gran impacto en el rendimiento del modelo. En esta etapa se realizan dos pasos que son: 1) transformación y 2) selección de variables [21].

En la etapa anterior (sección 2.2) se explicó cómo se recolectan los datos a través de un instrumento, obteniendo datos en bruto. Dependiendo del dominio del problema y la naturaleza de los datos, se deben aplicar funciones de preprocesamiento para que los datos en bruto se transformen en variables de entrada. Por ejemplo, si los datos son archivos de audio se pueden aplicar filtros para modificar la amplitud, la fase o longitud de onda [28]; o si son imágenes se pueden aplicar las funciones correspondientes para obtener el color, textura, resaltar borde, entre otras características [29]. En el caso de que sean valores numéricos, usualmente se utilizan funciones de agregación como, por ejemplo, promedio, mínimo, máximo, mediana, entre otras [21].

Luego de que los datos en bruto son transformados en variables, el siguiente paso es seleccionar las variables con mayor relevancia. La selección de variables es el proceso de obtener las características más importantes de un conjunto de datos. Los beneficios de aplicar alguna técnica de selección de variables son: la disminución del tiempo de entrenamiento, reducción de la complejidad del modelo y a su vez el sobreajuste, mejoramiento de la exactitud del modelo, entre otros. Esto se realiza porque en el conjunto de datos puede tener variables redundantes, irrelevantes o que contengan ruido; y en el proceso selección de variables, serían descartadas. Además, puede darse el caso que se tenga un conjunto de datos excesivamente grande, el cual hace difícil su 
manipulación, y lo que se lograría en este paso es la reducción de las dimensiones necesarias para entrenar el modelo [20]. Cabe indicar que la variable de salida o clase debe de estar definida, tal como se describirá en la sección 2.4.1.1.

Existen tres métodos de selección de variables que pueden ser utilizados para este fin, como lo son: métodos de filtro, envoltura y embebidos [30]. El método de filtro lo que hace es buscar si existe correlación de las variables de entrada con la variable de salida o clase. En caso de que la correlación sea baja, se elimina la variable de entrada del conjunto de datos. Este método tiene la ventaja de ser de rápida ejecución, además de disminuir la complejidad del modelo y reducir la posibilidad de que el modelo este sobreajustado. Sin embargo, una de las desventajas que tiene este método es que no toma en consideración la relación entre variables de entrada, es decir, este método considera que las variables de entrada son independientes, y como consecuencia se podrían tener variables redundantes en el conjunto de datos. Entre las técnicas más utilizadas están: correlación de Pearson, ANOVA, ChiCuadrado, entre otras [31].

Por otro lado, el método de envoltura entrena un modelo computacional múltiples veces usando diferentes combinaciones de variables de entrada, luego se comparan los resultados del modelo a través de validación cruzada y se decide que variables de entrada se debe agregar o eliminar. La ventaja de este método es que presenta mejores resultados en la etapa de evaluación de modelos. Sin embargo, se aumenta el riesgo de que el modelo tenga sobreajuste si las observaciones no son suficientes, además de tomar un tiempo significativo al utilizar este método; ya que se deben de entrenar todas las combinaciones posibles entre las variables de entrada. Entre las técnicas más utilizadas están la selección hacia adelante, hacia atrás y recursiva [30].

Finalmente, los métodos embebidos son similares a los métodos de envoltura, porque tratan optimizar el rendimiento de un modelo. La diferencia radica en que incorporan la selección de variables como parte del entrenamiento del modelo para reducir el tiempo de procesamiento. Entre las técnicas más utilizadas están: bosques aleatorios, métodos de regularización, arboles de decisión, entre otras [31]. 


\subsection{Construcción del modelo computacional}

En esta etapa se establecen diferentes pasos que permitirán entrenar y mejorar el rendimiento del modelo. En la práctica, no existe una guía específica para la construcción de un modelo computacional debido a que cada algoritmo tiene sus propias características de configuración. Sin embargo, en esta sección se describen varios conceptos que se pueden utilizar para este fin. Esta sección empieza por describir diferentes métodos para definir las categorías o la clase, necesarias para crear un modelo computacional supervisado. Luego, se definen ciertos criterios a tomar en cuenta sobre las características de los datos, en especial cuando se trabajan con una cantidad limitada de datos. Finalmente, se abordan las pautas necesarias para entrenar un modelo computacional, como por ejemplo la selección de los hiperparámetros ajustados a cada modelo y cómo evitar el sobreajuste, es decir, cómo construir un modelo que sea capaz de generalizar su predicción con respecto a los datos provistos.

\subsubsection{Configuración del modelo computacional}

\subsubsection{Definición de categorías o clases}

Existen varias técnicas para definir las categorías o clases que se utilizarán para construir el modelo computacional supervisado. Esta tarea es crucial, porque si los datos están mal etiquetados, todo el proceso de implementación del modelo será incorrecto. El etiquetado de datos es un proceso en el que cada instancia del conjunto de datos se le añade una etiqueta, es decir la clase a la que cada instancia pertenece dependiendo del problema a tratar. Una instancia puede ser vista como una observación. Para este proceso no existe un consenso de cómo realizarlo, inclusive hay casos que cada trabajo propone una nueva forma de realizar el etiquetado de datos. En la literatura se encuentran dos técnicas de etiquetado, como la son el manual y automático [32].

El etiquetado manual se refiere a expertos del área etiquetan los datos, es decir, cada instancia es categorizada con etiquetas previamente definidas. Esta técnica se basa mucho en la percepción humana, por lo que se recomienda que dos o más expertos intervengan en este proceso. Por lo general esta técnica tiene una alta exactitud siempre y cuando se apliquen métodos para medir el acuerdo entre los expertos. Para esto, existen varios indicadores, uno de los más conocidos es el IRR (InterRater Reliablity - Este indicador mide el grado de 
acuerdo entre dos o más evaluadores que realizan una evaluación independiente sobre el mismo conjunto de datos [33]), y busca garantizar que el etiquetado sea de utilidad. Una vez que los datos han sido etiquetados se calcula el IRR. Un valor bajo del IRR corresponde a que los evaluadores tienen diferentes percepciones sobre la evaluación de los datos; y deberán de recurrir a otra ronda de etiquetado, hasta que los expertos lleguen a un acuerdo.

Para calcular el IRR existen diferentes pruebas estadísticas como, por ejemplo, la probabilidad conjunta de acuerdo, Kappa de Cohen, coeficiente de correlación intraclase (ICC, por sus siglas en inglés), entre otras. La selección de una de ellas depende de varios criterios como el número de evaluadores o el tipo de escala que se esté utilizando. Uno de los más utilizados es el ICC, el cual calcula la robustez con que datos de un mismo grupo se parecen entre sí. El ICC opera sobre datos estructurados como grupos, en lugar de datos estructurados como pares de observaciones, es decir acepta más de dos evaluadores. El rango del ICC puede estar entre 0.0 a 1.0, valores cercanos a 0 indican que existe poco grado de acuerdo entre los evaluadores, mientras valores cercanos a 1 , los evaluadores dieron puntuaciones similares a cada uno de los registros [34].

Una vez el IRR da un valor aceptable (por ejemplo, un ICC entre 0,75 y 1), se elige la etiqueta más utilizada por instancia (moda) o el promedio de los valores de la etiqueta (media), dependiendo del tipo de escala que se utilice para la clase. Esta selección de la clase será considerada el "valor de verdad" o "valor estándar" para el conjunto de datos.

Por otro lado, el etiquetado automático ocurre cuando un algoritmo no supervisado, como K-medias, $\mathrm{K}$ vecino más cercano, o clusterización jerárquica, añade etiquetas al conjunto de datos. Los algoritmos no supervisados buscan patrones en el conjunto de datos para luego agrupar los datos que tienen similitud entre ellos y asignar una etiqueta a cada grupo, dependiendo del problema que se esté tratando. Esta técnica puede resultar ventajosa porque es automática y rápida, pero puede tener poca exactitud al momento de etiquetar los datos, porque carece de una validación efectiva. 


\subsubsection{Características del conjunto de datos}

Para que un modelo computacional pueda aprender necesita un conjunto de datos adecuado. Debido a que los modelos computacionales son aplicados a problemas de la vida real, muchas veces los datos capturados tienen ciertas propiedades inherentes al problema a resolver, como por ejemplo el tamaño de datos o la distribución desequilibrada de las clases.

El tamaño del conjunto de datos influye mucho en el rendimiento del modelo computacional, puesto que, a mayor número de instancias, el modelo puede generalizar mejor su clasificación [22]. Sin embargo, debido a que no siempre se cuenta con una gran muestra de datos, se deben de considerar técnicas que nos permitan asegurar que el modelo a construir puede ser capaz de ser generalizable con menor cantidad de datos. Para este fin, se puede aplicar técnicas de sobremuestreo, la cual nos permite generar datos sintéticos, a partir del conjunto de datos inicial [35].

Por otro lado, la distribución desequilibrada de las etiquetas o clases puede convertirse en un problema sino se aplican las técnicas adecuadas para que el modelo aprenda esta característica del conjunto de datos. El conjunto de datos tiene una distribución balanceada cuando las clases del conjunto de datos tienen relativamente la misma distribución y tienen una distribución desbalanceada, cuando al menos una clase tiene una menor proporción. Esta distribución desbalanceada puede causar que el modelo computacional no sea generalizable, perjudicando a la clase con menor distribución. Existen varias estrategias para manejar la situación de datos desbalanceados. Primero, dependiendo del algoritmo a ser utilizado, se recomienda ajustar los hiperparámetros para que pueda aceptar esta propiedad de los datos. Segundo, se pueden aplicar técnicas de sobremuestreo que permitan generar muestras sintéticas y balancear la distribución. Adicionalmente, cuando se trata con una distribución desbalanceada hay que seleccionar las métricas adecuadas para evaluar el modelo computacional, ya que se pueden tener conclusiones erróneas si solo se considera la clasificación exitosa de la clase con mayor distribución [22]. Una explicación con más detalle se presenta en la sección 2.5. 


\subsubsection{Generación de datos sintéticos}

La generación de datos sintéticos consiste en incrementar la cantidad y diversidad de instancias en un conjunto de datos [35]. No se recopilan nuevos datos, sino que se transforman los datos ya adquiridos. Uno de los principales beneficios de utilizar técnicas para generar datos sintéticos es que puede actuar como regulador para prevenir el sobreajuste de un modelo y mejorar el rendimiento en problemas que utilizan clases no balanceadas [36]. En la teoría de aprendizaje automático, esta técnica también se la llama sobremuestreo, el cual, utilizando métodos estadísticos, crean nuevas instancias sintéticas en un conjunto de datos. Existen varias técnicas para hacer sobremuestreo en un conjunto de datos, siendo tres técnicas las más utilizadas: 1) minoría sintética (SMOTE, por sus siglas en inglés), 2) muestreo sintético adaptivo (ADASYN, por sus siglas en inglés) y 3) sobremuestreo aleatorio [37].

EI SMOTE toma un subconjunto de las características de una instancia y de sus vecinos más cercanos. Luego, el algoritmo genera nuevas instancias combinando las características del dato seleccionado con las de sus vecinos [38]. Este algoritmo recepta dos parámetros, la cantidad de instancias que se desea crear y cuantos vecinos toma en consideración para combinar los datos. Dependiendo de la cantidad de datos se recomienda tomar entre 2 a 5 vecinos [38]. ADASYN es una extensión de SMOTE con la diferencia que trata de crear más puntos en los límites de la clase que en el interior de esta. Con esto se puede reducir el sesgo que tiene el conjunto de datos desbalanceado y reforzar los límites de cada clase para que el modelo computacional pueda predecir mejor [39]. El sobremuestreo aleatorio implica que se eligen observaciones de una clase en particular y se la duplica aleatoriamente en el conjunto de datos. Esta técnica puede balancear un conjunto de datos, pero aumenta la probabilidad de sobreajuste del modelo y puede descartar observaciones útiles [35].

\subsubsection{Entrenamiento del modelo computacional}

Para entrenar un modelo computacional primero se debe de elegir el algoritmo que más se ajuste al problema a resolver. Luego, utilizando el conjunto de datos se procederá a entrenarlo, de tal manera que su predicción aumente a medida que se va ajustando a los datos. El entrenamiento no solo depende del algoritmo elegido, sino también de la configuración de los hiperparámetros de 
cada modelo, así como también del conjunto de datos utilizado para entrenamiento, prueba y validación, cuya distribución dependerá del tamaño del conjunto de datos [40]. Finalmente, una vez entrenado el modelo se debe de analizar si el modelo es generalizable o si existe un sobreajuste. Cabe recalcar que todos estos pasos son realizados iterativamente hasta que se logre tener un modelo adecuado con un rendimiento aceptable. A continuación, se detallan estos procesos antes mencionados que permitirán entrenar el modelo computacional, así como también se presentan los fundamentos teóricos de diferentes modelos computacionales.

\subsubsection{Selección del modelo computacional}

Existen diversos modelos computacionales que se pueden utilizar dependiendo del problema a solucionar y del conjunto de datos seleccionado. Los modelos computacionales en su gran mayoría utilizan algoritmos de inteligencia artificial para analizar los datos. La inteligencia artificial se puede definir como la capacidad de un sistema de interpretar datos, aprender de ellos y aplicar el conocimiento aprendido en un problema determinado [41]. La inteligencia artificial se puede dividir de acuerdo con las técnicas que se utilice para el tratamiento de los datos, aprendizaje, exploración o descubrimiento de conocimiento y razonamiento sobre los datos [42].

El aprendizaje sobre los datos se refiere a la implementación de algoritmos de aprendizaje, los cuales tienen como objetivo aprender automáticamente sin la intervención de las personas. Los algoritmos de aprendizaje más conocidos son: ANN, SVM, y RF. En la exploración o descubrimiento de conocimiento a través de los datos, el principal objetivo es la identificación de información útil, valida y comprensible para que luego pueda ser convertida en conocimiento. Entre los algoritmos más utilizados están el Kmedias, K-vecinos más cercanos, clusterización jerárquica, entre otros. Finalmente, el razonamiento de datos involucra el uso de técnicas de lógica como deducción e inducción para generar conocimiento a través los datos. Los sistemas que utilizan técnicas de razonamiento buscan ejecutar tareas simulando a un experto de una manera especializada modelando el dominio de interés. Los algoritmos más utilizados incluyen el razonamiento basado en reglas, el razonamiento basado en casos y la lógica difusa. A continuación, se 
describirá la teoría de los modelos computacionales se seleccionados para este estudio. Como se mencionó en la sección 1.1, los modelos computacionales más utilizados para identificar el estilo de conducción son: la lógica difusa, las máquinas de soporte vectorial, las redes neuronales artificiales, los k-vecinos más cercanos y los bosques aleatorios.

\section{a. Lógica difusa}

La lógica difusa es una rama de la Inteligencia Artificial (IA), que se caracteriza por considerar la incertidumbre en los datos, al agregar conceptos verdaderos y falsos de la lógica común [43]. Para diseñar un modelo de lógica difusa, se deben seguir tres pasos: 1) fuzzificación: esta etapa define las funciones de pertenencia y las variables lingüísticas de las entradas. 2) Reglas de evaluación: en esta etapa se aplican las reglas de lógica difusa para calcular la salida; y, 3) defuzzificación: donde finalmente, un sistema de inferencia difusa (FIS, por sus siglas en inglés) convierte la salida en un resultado entendible [44]. Los dos tipos más utilizados de FIS son el modelo borroso Takagi-Sugeno (Sugeno) y el sistema de inferencia difusa Mamdani. Si bien Mamdani puede capturar mejor la experiencia humana, Sugeno es más eficiente computacionalmente que Mamdani [45, 46].

\section{b. Red neuronal artificial}

La red neuronal artificial o ANN (por sus siglas en inglés) es un sistema computacional, diseñado para simular sistemas de aprendizaje biológicos como el cerebro humano, y se utiliza para reconocer patrones, agrupar datos y clasificar datos etiquetados. La ANN está compuesto de neuronas las cuales se encuentran interconectadas y operan multiplicando cada entrada de la neurona con el peso de la conexión. Estos productos se suman utilizando una función de propagación para generar la salida de la neurona [47]. Las ANN pueden aprender elementos complicados de procesamiento de información no lineal. Estos modelos computacionales se ha utilizado en campos como visión por computadora [48], reconocimiento de voz [49], diagnóstico de enfermedades [50], entre otros. Un aspecto importante de las ANN es su capacidad autónoma de aprender de un conjunto de datos, ya que puede ser entrenado en modo supervisado o no supervisado. La forma simple de una red neuronal supervisada es el perceptrón multicapa (MLP, por sus siglas en inglés) y consta de al menos 
tres capas: la capa de entrada, la capa oculta y la capa de salida. EI MLP utiliza la técnica de propagación "back propagation" para entrenar la red neuronal. Este proceso compara cada salida de la neurona con la salida esperada, y se realiza de atrás hacia adelante, los pesos de cada neurona se ajustan hasta que el error de la red neuronal se minimiza o alcanza el criterio de parada [51].

\section{c. Máquinas de soporte vectoriales}

Las máquinas de soporte vectoriales (SVM, por sus siglas en inglés) es un modelo de aprendizaje supervisado que se ha aplicado en problemas de clasificación y regresión y fue introducido en 1992 por Boser, Guyon y Vapnik [52]. Este modelo ha sido ampliamente probado en muchas aplicaciones como reconocimiento de voz [53], detección de texto e hipertexto [54], procesado de imagen [55] entre otros. La idea del SVM es encontrar el hiperplano óptimo que separe las clases por las distancias máximas posibles. Esta separación se puede hacer en el mismo espacio de características, o para problemas más complejos, los datos utilizan una función kernel para trazar un mapa en un plano de alta dimensión para facilitar la clasificación [56]. El estudio presentado por Noble [57] demostró que cualquier conjunto de datos se puede separar linealmente por la función kernel correcta, pero identificar esta función es una caja negra, solo se puede hacer por prueba y error. Las funciones kernel más utilizadas son sigmoide, función de base radial (RBF), lineal y polinomial. En el caso de que una proporción del conjunto de datos este clasificado erróneamente, el usuario puede modificar el parámetro $C$ que permite ajustar la compensación entre el margen y el error de ajuste, a este concepto se le conoce como el "margen blando".

d. Bosques aleatorios

El algoritmo de bosques aleatorios (RF por sus siglas en inglés) fue propuesto por Breiman [58], y es un método de aprendizaje conjunto utilizado para la clasificación o regresión. Este algoritmo se implementa mediante la creación de un conjunto de árboles de decisión, y juntos votan para obtener un resultado. El RF se ha aplicado en muchos ámbitos de investigación como la bioinformática, predicción de abandono de clientes, o clasificación de imágenes aéreas [59]; debido a su alta precisión y capacidad para manejar características extensas con muestras pequeñas. Este algoritmo aplica dos conceptos: bagging 
y selección aleatoria de características [60]. El bagging implica tomar muestras repetidas de un conjunto de datos; y se usa para entrenar árboles de decisión basándose en la idea de que la combinación de modelos de aprendizaje mejora el resultado. Por otro lado, la selección de características divide cada nodo en un subconjunto aleatorio del conjunto original de características para construir un conjunto de árboles de decisión con varianza controlada. Hay dos parámetros para tener en cuenta en la configuración de un RF: la cantidad de árboles que crecen en el bosque y la cantidad de características. La optimización de los parámetros se puede realizar mediante una búsqueda en cuadrícula (grid search en inglés) como se explicará en la sección 2.4.2.2.

\section{e. K-vecinos más cercanos}

El k-vecinos más cercanos (kNN por sus siglas en inglés) es un método no paramétrico utilizado para la clasificación y la regresión, y posiblemente sea el más simple de todos los enfoques de clasificación supervisados. Este algoritmo se basa en la idea de que las observaciones (puntos) con características similares generan resultados similares [61]. Hay dos parámetros a considerar para construir un modelo kNN: el número de vecinos $(\mathrm{k})$ y la distancia entre los puntos que usualmente usan la función de distancia euclidiana. Para la selección de los $k$ vecinos se pueden considerar valores pequeños o grandes. Los valores pequeños eligen los puntos de entrenamiento más cercanos a un punto, y se ha demostrado que puede estimar mejor la clasificación correcta en el punto de prueba. Sin embargo, debido a la consideración de pocos ejemplos, esta estimación es propensa a fluctuaciones estadísticas. Por el contrario, los valores grandes de $k$ reducen los errores estadísticos, pero permiten que los puntos lejanos contribuyan a la generalización de la clase, lo que puede suavizar algunos de los detalles de las distribuciones de clase. Por lo general, $k$ se elige dependiendo del valor que minimice el error de clasificación en algunos datos de validación independientes o mediante procedimientos de validación cruzada.

\subsubsection{Configuración de hiperparámetros}

La configuración de hiperparámetros es un proceso de prueba y error que trata de encontrar los valores óptimos donde el modelo tiene mejor rendimiento sin que esté sobreajustado. Los hiperparámetros son parámetros o valores de configuración del modelo y se modifican en el proceso de aprendizaje. Cada 
algoritmo de aprendizaje tiene sus propias recomendaciones de cómo se deben configurar los hiperparámetros, tal como se describió en la sección anterior. En la literatura, existen tres técnicas generales que pueden ser utilizadas en la configuración de los hiperparámetros, como lo son la búsqueda cuadriculada (grid search), búsqueda aleatoria (random search) y técnicas de optimización [21].

La búsqueda cuadriculada construye modelos con cada combinación posible de valores de hiperparámetros, para luego evaluar cada modelo y seleccionar el que tenga mejor rendimiento. Por otro lado, el método de búsqueda aleatoria es similar al de búsqueda cuadriculada, pero requiere menos tiempo porque no prueba todas las combinaciones posibles. En este caso, se proporciona un conjunto de valores por cada hiperparámetros, de la cual se toma los valores al azar hasta cumplir un número de iteraciones. Luego se evalúa cada modelo y se selecciona el mejor [21]. Otra opción diferente a la búsqueda cuadriculada o búsqueda aleatoria es utilizar algoritmos de optimización (por ejemplo, optimización bayesiana o algoritmos genéticos). Los algoritmos de optimización se diferencian de la búsqueda cuadricula o aleatoria porque utilizan la evaluación resultante de un conjunto de hiperparámetros para elegir los siguientes valores de estos. En resumen, el algoritmo de optimización reduce el número de veces que se debe entrenar y evaluar un modelo, eligiendo los mejores valores para los hiperparámetros, tomando en consideración los valores anteriores que dieron buenos resultados. Por lo general, se debe tener en consideración dos aspectos en los algoritmos de optimización: a) la función sustitutiva, que es la que se encarga de encontrar el comportamiento de la función objetivo y asigna los nuevos valores a los hiperparámetros; b) la función de pérdida, que es la que se encarga de evaluar el rendimiento de los nuevos valores de los hiperparámetros. Este proceso se repite hasta que el valor de evaluación llegue a un valor óptimo [62].

\subsubsection{Sobreajuste}

Se denomina sobreajuste cuando se sobreentrena un modelo computacional y no es capaz de generalizar los resultados. En otras palabras, sobreajuste es cuando el modelo se ajusta mayormente a las características del conjunto de datos de entrenamiento, evitando la capacidad del modelo de predecir 
correctamente nuevos datos. Para evitar el sobreajuste existen algunas técnicas, dependiendo del modelo que se esté entrenando. Por lo general las técnicas más utilizadas son: a) regularización y b) parada anticipada.

La regularización busca realizar ligeras modificaciones en el algoritmo para que el modelo tenga una mejor generalización. La regularización agrega un término que penaliza a la complejidad del modelo y a su vez minimiza la función de costo. Comúnmente se utilizan las técnicas de regularización Lasso (L1), regularización Ridge (L2) y malla elástica [63]. La regularización Lasso (L1), es una técnica que selecciona algunas características (variables de entrada) para minimizar a cero, de esta manera no tendrán incidencias en el modelo. En la literatura se encuentra que L1 es una técnica para seleccionar las características, debido que el modelo solo tomará en cuenta las variables que son más influyentes. Esta técnica se utiliza cuando las variables de entrada no están correlacionadas entre ellas.

La regularización Ridge (L2) lo que hace es suavizar el peso de todas las características, para que sean de menor influencia para el modelo. Esta técnica se utiliza cuando las variables de entrada están correlacionadas entre sí. Finalmente, la regularización de malla elástica combina las técnicas de L1 y L2 y es recomendable utilizarla cuando en el conjunto de datos está compuesto de muchas variables de entrada, porque algunas de ellas serán irrelevantes, y otras están correlacionadas entre ellas [22].

Por otro lado, la parada anticipada (early stopping) es otra técnica utilizada para evitar el sobreajuste del modelo. La parada anticipada generalmente se utiliza en modelos que se entrenan de forma iterativa, en donde se delimita un número determinado de iteraciones, como, por ejemplo, una red neuronal puede ser entrenada en 500 iteraciones. Para aplicar esta técnica, es necesario definir un conjunto de datos de entrenamiento, y de validación. También, es necesario seleccionar un número grande para las iteraciones, de forma que se pueda observar que el modelo se ha estabilizado. Adicionalmente, se necesita definir una métrica de evaluación (p. ej. el error, o la exactitud) para determinar cuándo el modelo debe de parar anticipadamente. Una vez que el modelo ha sido entrenado con sus datos de entrenamiento, se valida el modelo con los datos de validación. Es aconsejable graficar los valores de la métrica de evaluación 
escogida del entrenamiento y validación para observar cuándo se debe de realizar la parada anticipada. Cuando el error de entrenamiento empieza a disminuir y estabilizarse; y al mismo tiempo empieza a incrementar el error de validación, significa que el modelo ha empezado a sobre ajustarse a los datos, por lo que se debe de realizar la parada anticipada. Es decir, el modelo debe de parar de entrenar cuando el error de validación llega a su punto mínimo [64].

\subsection{Evaluación del modelo computacional}

La selección de métricas de evaluación no es un proceso sencillo, se deben tener en consideración varios aspectos como por ejemplo el objetivo del estudio o si el conjunto de datos esta balanceado [21]. Esta sección describe algunas de las medidas más utilizadas que se han aplicado en la evaluación de modelos computacionales, como lo son las métricas derivadas de la matriz de confusión y las pruebas de significancia estadísticas.

\subsubsection{Métricas de la matriz de confusión}

La matriz de confusión es una tabla que se genera a partir de los resultados obtenidos por el modelo y por el otro los resultados de los "valores de verdad" o clase, como se describió en la sección 2.4.1.1. Como se puede observar en la Figura 2.2, los valores que puede tomar la matriz de confusión son verdaderos positivos (VP), verdaderos negativos (VN), falsos positivos (FP) y falsos negativos (FN) [24].

\section{PREDICCIONES}

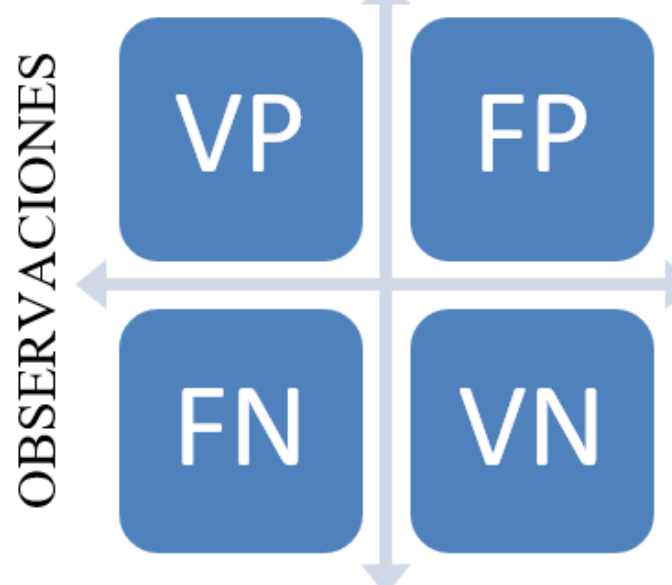

Figura 2.2 Matriz de confusión 
- VP es la cantidad de instancias positivas que fueron etiquetadas correctamente como positivas por el modelo.

- VN es la cantidad de instancias negativas que fueron etiquetadas correctamente como negativas por el modelo.

- FN es la cantidad de instancias positivas que fueron etiquetadas incorrectamente como negativas.

- FP es la cantidad de instancias negativas que fueron etiquetadas incorrectamente como positivas.

A partir de los valores de la matriz de confusión se pueden obtener diferentes métricas de rendimiento como, por ejemplo, exactitud, precisión, especificidad, entre otras. A continuación, se detalla cada una de ellas [20]:

La exactitud es la métrica más usada en trabajos de investigación. Sin embargo, hay que tomar en consideración que esta métrica funciona bien cuando se tiene una distribución balanceada en el conjunto de datos. Esta métrica se define como la cantidad de predicciones correctas, sobre el total de datos.

$$
\text { exactitud }=\frac{V P+V N}{V P+V N+F P+F N}
$$

La métrica precisión es la relación entre las instancias que tuvieron una predicción correcta (VP) y el total de instancias pronosticadas por el modelo, tanto correctas (VP) como incorrectas (FP).

$$
\text { precisión }=\frac{V P}{V P+F P}
$$

La métrica de sensibilidad es la relación entre los casos verdaderos positivos (VP) y la suma de los verdaderos positivos y falsos negativos. En otras palabras, es la fracción de instancias positivas que fueron correctamente clasificadas por el modelo.

$$
\text { sensibilidad }=\frac{V P}{V P+F N}
$$

La métrica de especificidad es la relación entre los casos verdaderos negativos (VN) y la suma de los falsos positivos y verdaderos negativos. En otras palabras, es que tan bien el modelo predice que una instancia no pertenece a una clase en particular.

$$
\text { especificidad }=\frac{V N}{F P+V N}
$$


La métrica de Medida- $F$ ( $F$-Score en inglés) es también muy usada porque combina la precisión y sensibilidad en un solo valor, y ofrece una perspectiva general del rendimiento del modelo. Se define como el doble del producto de precisión y sensibilidad sobre la suma de estos dos.

$$
F-\text { Score }=\frac{2 *(\text { precisió } n * \text { sensibilidad })}{\text { precisión }+ \text { sensibilidad }}
$$

Otras métricas utilizadas para evaluar el rendimiento de un clasificador cuando el conjunto de datos esta desbalanceado son: el índice de kappa y el área bajo la curva (area under the curve - AUC) [24, 65]. El índice de kappa (K) es una medida que compara la precisión entre los datos observados (muestra) y los valores correspondientes a las predicciones [65]. Este índice da valores entre 0 a 1 , donde 1 significa una concordancia perfecta y 0 una concordancia insignificante. Landis y Koch propusieron una escala de valores en el cual describe el grado de acuerdo, como se observa en la Tabla 2.2 [66].

\begin{tabular}{cc}
\hline Rango de valores & Descripción \\
\hline $0<\mathrm{K}<0.20$ & Insignificante \\
$0.20 \leq \mathrm{K}<0.40$ & Discreta \\
$0.40 \leq \mathrm{K}<0.60$ & Moderada \\
$0.60 \leq \mathrm{K}<0.80$ & Sustancial \\
$0.80 \leq \mathrm{K}<1.00$ & Casi perfecta
\end{tabular}

Tabla 2.2 Rango de valores para interpretar el indica kappa.

Por otro lado, el área bajo la curva (AUC) es una medida que considera la curva ROC (Receiver Operating Characteristic), la cual mide la capacidad del clasificador de distinguir entre clases. Una curva ROC es un gráfico bidimensional representando a la métrica de sensibilidad en el eje $X$ y 1 especifidad en el eje Y. La métrica de AUC devuelve el área de la curva ROC, tomando valores entre 0 a 1 ; siendo 1 un clasificador perfecto y 0 un clasificador con bajo rendimiento [24]. Hosmer y Lemeshow sugirieron los rangos de valores que se puede considera como mal y excelente clasificador. En la Tabla 2.3 se puede observar los rangos de valores con sus descripciones [67]. 


\begin{tabular}{ll}
\hline Rango de valores & Descripción \\
\hline AUC $=0.5$ & No hay discriminación \\
$0.5<$ AUC $<0.7$ & Discriminación mala \\
$0.7 \leq$ AUC $<0.8$ & Discriminación aceptable \\
$0.9 \leq A \cup C<0.9$ & Discriminación excelente \\
AUC $\geq 0.9$ & Discriminación excepcional \\
\hline Tabla 2.3 Rango de valores para interpretar la métrica AUC
\end{tabular}

\subsubsection{Pruebas estadísticas}

Otra forma de evaluar un modelo es a través de pruebas estadísticas para validar la significancia de las predicciones de un modelo computacional, es decir, evalúa que los resultados de las métricas de evaluación no fueron obtenidos por casualidad. También, estas pruebas demuestran si existe diferencia significativa entre modelos, de lo contrario, no se puede asumir que los modelos se comportan de forma distinta [24].

Desde la perspectiva estadística, para aplicar estas pruebas se debe establecer una hipótesis nula $\left(\mathrm{H}_{0}\right)$ que se trata de refutar. Generalmente, la $\mathrm{H}_{0}$ confirma o descarta si la evaluación y el comportamiento es igual para todos los modelos. Si la hipótesis se rechaza, se infiere que el modelo que tiene mejor rendimiento es mejor que el otro modelo [40].

Para seleccionar la prueba estadística que mejor se ajuste al conjunto de datos es necesario tomar en consideración la distribución de los datos y el tamaño de la muestra. Por ejemplo, se pueden utilizar pruebas paramétricas si el conjunto de datos tiene una distribución normal, el tipo de variable de tipo continua o se tiene una muestra grande de datos. Caso contrario, se debería de utilizar una prueba no paramétrica, ya que esta considera una distribución asimétrica, una muestra pequeña de datos 0 ; se tiene una variable de tipo ordinal o categórica [68].

Debido a que el problema a tratar en este trabajo cuenta con una variable categórica ( $p$. ej. estilo de conducción normal, agresivo) y generalmente se obtiene un conjunto pequeño de datos, las pruebas no paramétricas son la mejor opción. Entre las pruebas no paramétricas más utilizadas están la prueba de 
McNemar, Wilcoxon y Friedman. Para esta investigación vamos a utilizar la prueba Wilcoxon debido a que los datos no siguen una distribución específica y la variable es ordinal. La prueba de Wilcoxon compara el rango medio de conjuntos de datos y determina si existe diferencias significativas entre ellas. En otras palabras, debido a que las dos muestras proceden de la misma población, al ordenar las observaciones de menor a mayor, se espera que estas observaciones se distribuyan en forma simétrica, esto quiero decir que no haya diferencias entre los conjuntos de datos. Por lo contrario, si se ordena los conjuntos de datos y una población tiene valores mayores o menores que la otra, estas no se distribuirán de forma simétrica, por lo tanto, si hay diferencias significativas entre los conjuntos de datos [69].

\subsection{Resumen}

En este capítulo se describieron los fundamentos teóricos y prácticos necesarios para el proceso de implementación de un modelo computacional, el cual fue definido en seis etapas. Cada etapa debe de considerarse crucial para la implementación de un modelo. Tal como se ha descrito en cada etapa, la selección de las diferentes técnicas varía dependiendo del problema a tratar. En este trabajo en particular, en donde se implementarán varios modelos computacionales para la identificación del estilo de conducción hay que tomar en cuenta algunas propiedades inherentes de los datos como lo son: 1) el tamaño del conjunto de datos, 2) la distribución desbalanceada de datos y el 3) tipo de etiqueta o clase, en este caso, ordinal. Teniendo en cuenta estas propiedades, se podrán seleccionar las técnicas que se ajusten a este problema, las cuales serán detalladas en el capítulo 4.

A continuación, el siguiente capítulo revisa la literatura correspondiente a la identificación de los estilos de conducción, con el propósito de tener una idea general sobre estado del arte en estas investigaciones y así poder proporcionar una solución a los desafíos encontrados en esta literatura. 


\section{Capítulo 3: Estado del Arte del Estilo de Conducción}

En este capítulo se presentan los fundamentos teóricos y trabajos previos relacionados con el estilo de conducción. Principalmente, esta sección proporciona la definición de estilos de conducción, así como los factores que influyen en ésta y los dos enfoques más utilizados para identificar y analizar el estilo de conducción. Luego, esta sección presenta la revisión de trabajos previos que abarcan la clasificación automática del estilo de conducción, tomando en cuenta: 1) la forma en que se recolectan los datos, 2) las variables de entrada usadas, 3) las etiquetas usadas para la clasificación y 4) los modelos computacionales implementados. Finalmente, este capítulo describe las limitaciones y desafíos derivados de trabajos previos con relación a los modelos computacionales ya existentes.

\section{1 ¿Qué es el estilo de conducción?}

El área de conducción segura ha prestado considerable atención al estudio de los estilos de conducción, debido a que se encuentra estrechamente relacionado con los accidentes de tránsito. Según Elander et al. [1], los estilos de conducción se conocen como "la forma en que las personas eligen conducir o los hábitos de conducción que se han establecido a lo largo de los años, incluida la elección de la velocidad de conducción, el umbral para adelantar, avanzar y la propensión a cometer infracciones de tránsito" (Elander, 1993, página 279).

Sagberg et al. [70] señaló en un estudio reciente que el estilo de conducción es diferente para cada individuo y éste representa el comportamiento de un conductor durante un período, es decir, el comportamiento de un conductor que viaja desde un punto $A$ hacia un punto $B$. El mismo autor propone que el estilo de conducción se puede establecer como un proceso de reforzamiento de los hábitos de los conductores determinados por diferentes factores, como lo son [70]: 
1. Factores demográficos/socioculturales y tecnológicos: Estos factores pueden ser características del individuo y del entorno de éste. Entre las características más investigadas están: el género, edad, experiencia manejando, cultura social, entre otras; y tecnológicas (p. ej. características del vehículo, sistemas de soporte).

2. Factores de contexto y/o externos: El estilo de conducción puede ser influenciado por situaciones externas en las que el conductor se expone al momento de manejar, por ejemplo, el flujo del tráfico, tipo de camino, hora del día, clima, entre otros.

3. Factores humanos: El comportamiento del conductor puede estar relacionado con su personalidad (p. ej. enojo, ira, paciencia, ansiedad, etc.), estado motivacional (p. ej. estrés, cansancio, competencia), habilidades al manejar (p. ej. experiencia) o, también motivos secundarios como querer llegar más rápido a su destino.

Estos tres factores determinan directa o indirectamente el comportamiento del conductor. En el caso de los factores humanos, varios trabajos han asociado los comportamientos de imprudencia, descuido, ansiedad, enojo y hostilidad con los factores demográficos y socioculturales, como el género, edad y el nivel educativo [71-73]. Estos trabajos reportaron que las mujeres se estresan más (altos niveles de ansiedad) mientras conducen, que las personas mayores conducen con más calma (bajos niveles de ira) [71], y que los hombres conducen de manera agresiva (altos niveles de imprudencia y enojo) [73]. Además, no se encontró una relación entre el estilo de conducción y los factores socioeconómicos de los conductores (p. ej. educación, salud, estado familiar). Otro estudio realizado a conductores profesionales, reveló una correlación positiva de los niveles de ira con el estilo de conducción agresiva (ansiosos y enojados) [72].

Por otro lado, algunas investigaciones han reportado cómo el contexto o la situación, influye en el comportamiento del conductor. El estudio presentado por Ericsson [74] exploró cómo el flujo de tráfico (bajo, medio y alto), el tipo de carretera (urbana, rural), así como la hora del día (durante el día o la noche) influyeron en el comportamiento del conductor. Por ejemplo, un conductor podría exhibir un comportamiento agresivo mientras conduce en la ciudad o durante las 
horas pico (p. ej. manejar a alta velocidad); o cuando una carretera está menos transitada, el conductor puede exponer un estilo calmado (p. ej., conducir a velocidades más bajas). Kilpelainen y Summala hicieron una investigación en Finlandia [75], en la cual realizaron una encuesta a 1437 personas sobre su percepción del clima y que decisiones tomaron de acuerdo con la información de la misma. Este estudio encontró que cuando las personas manejan en malas condiciones de clima, especialmente cuando nieva, los conductores disminuyen la velocidad entre 6 a $7 \mathrm{~km} / \mathrm{h}$.

Hay varias etiquetas o clases que se han definido para determinar el estilo de conducción, las cuales tratan de medir el grado de agresividad que tiene el conductor. En la revisión de literatura presentada por Martinez et al., se determinó que las clases más utilizadas son calmado y agresivo [7], siendo la categoría de agresivo la más relevante, debido a su relación con los accidentes de tránsito. A continuación, se detalla el estilo de conducción agresivo, el cual va a ser estudiado a lo largo de este trabajo.

\subsection{Estilo de conducción agresivo}

Como se mencionó en la sección anterior, una de las principales causas de accidentes de tránsito es conducir de manera agresiva [2, 76]; esto conlleva a pérdidas millonarias, lesiones en las personas o inclusive la muerte [3]. Debido a esto, es la importancia de este término en las investigaciones de estilo de conducción. Este tipo de conducción se puede considerar como un mal hábito o un comportamiento riesgoso cuando se maneja. La mayoría de las investigaciones relacionadas al estilo de conducción tratan de identificar al conductor que maneja de manera agresiva.

En la literatura se puede encontrar varios significados para la conducción agresiva. La más utilizada entre los investigadores es la propuesta por Tasca [77], el cual definió que este comportamiento puede aumentar el riesgo de un choque y está estimulado por la impaciencia, la frustración o ira y/o un intento de ahorra tiempo. Otro concepto similar ha sido propuesto por Neuman et al. [78], puntualizando que la conducción agresiva es manejar un coche de manera egoísta, enojada o impaciente, a menudo de forma insegura, afectando a otros conductores. También hay otros conceptos con una tendencia más marcada en 
la seguridad vial, refiriendo al estilo de conducción agresivo como eventos inseguros que realizan los conductores agresivos, como por ejemplo, las infracciones de tránsito o realizar maniobras inseguras al manejar, pasar los semáforos en rojo, no tener distancia con el carro al frente, manejar zigzagueando, entre otros [79].

\subsection{Métodos para determinar el estilo de conducción}

En la literatura se ha encontrado que los dos enfoques más utilizados para determinar el estilo de conducción son [80]: 1) la autoevaluación a través de cuestionarios, enfocándose en las motivaciones y emociones del conductor y 2) el análisis de datos del vehículo, el cual se hace a través de pruebas de manejo y hace énfasis en las maniobras que realiza el conductor.

\subsubsection{Cuestionarios de autoevaluación}

La autoevaluación recopila comportamientos del conductor a través de cuestionarios y es ampliamente utilizado debido a su bajo costo y facilidad de aplicación. Por lo general, este método trata de encontrar una correlación entre los accidentes de tránsito y factores humanos; como por ejemplo los estados emocionales del conductor (p. ej. ira, irritación, frustración), estados motivacionales ( $p$. ej. aburrimiento, competencia, estrés) y la información demográfica o sociocultural [81-83].

Uno de los cuestionarios de autoevaluación utilizados a nivel mundial para medir el comportamiento del conductor es el "Cuestionario de Comportamiento del Conductor" (DBQ, por sus siglas en inglés). El DBQ es un instrumento que contiene 50 preguntas, las cuales se dividen en 3 componentes que son: infracciones, errores y lapsos. Las infracciones son actos de incumplimiento a la ley, ejemplos comunes son: exceso de velocidad y pasar la luz roja. Los errores son actos que no logran el resultado previsto como activar el limpia parabrisas en vez de encender las luces delanteras. Por último, los lapsos son actos no intencionados como olvidar la ruta seleccionada o dejar las llaves dentro del coche [84]. Dicho cuestionario tiene como objetivo medir el grado de relación entre estos tres componentes y los accidentes de tránsito [81]. Estudios previos han reportado que existe una correlación entre los tres componentes del DQB y los accidentes de tránsito, indicando que los accidentes de tránsito están 
asociados principalmente con las características de la persona como la edad, el sexo y la ubicación geográfica. En particular, los resultados demostraron que las infracciones de tránsito disminuyeron con la edad; que los errores y la propensión a cometer infracciones es mayor en los hombres que en las mujeres; y que las mujeres obtuvieron altos puntajes en el componente de lapsos [81, 85].

En la literatura se encuentra también otro cuestionario de autoevaluación llamado "Inventario de Comportamiento del Conductor" (DBI, por sus siglas en inglés) [82], el cual ha sido utilizado para medir el estrés del conductor. Estudios previos utilizando el DBI han sugerido tres efectos en el comportamiento de la persona por el aumento del estrés al manejar: agresión (es decir, ira, impaciencia y asunción de riesgos), enojo al conducir (es decir, ansiedad, y falta de confianza) y estado de alerta (es decir, conciencia de riesgo y búsqueda activa de peligros viales) [86, 87].

Por otro lado, Deffenbacher et al. [88] desarrollaron el cuestionario denominado "Escala de la Ira al Conducir" (DAS, por sus siglas en inglés) para estudiar otro estado emocional del conductor de gran importancia, como lo es la ira. Este cuestionario trata de predecir el enojo que un conductor tiene hacia otros conductores, la impaciencia que se manifiesta en avenidas con tráfico, la conducción agresiva y arriesgada e infracciones de tránsito. Manejar mientras el conductor está enojado puede incrementar la probabilidad de comportamientos riesgosos, como aumentar la velocidad, comportamientos agresivos verbales e inclusive hasta comportamientos físicos [88]. En un estudio realizado a 1500 estudiantes universitarios utilizando el DAS, encontraron que los hombres estaban más enojados por la presencia de la policía y por experimentar períodos de conducción lenta; mientras que las mujeres estaban más enojadas por el comportamiento ilegal y las obstrucciones del tráfico, pero no hubo diferencias de género en la puntuación total de dicho cuestionario [83].

En resumen, los cuestionarios aplicados a conductores y utilizados en varios estudios han demostrado que existen varios comportamientos que pueden determinar el estilo del conductor. Sin embargo, un problema importante con los cuestionarios es que la información obtenida es subjetiva, y la mayoría de las veces no representa un comportamiento preciso del conductor [89, 90]. 


\subsubsection{Análisis de datos del vehículo}

Por otro lado, varias investigaciones han optado por utilizar datos del vehículo para analizar y relacionar estos datos con varios eventos de conducción (p. ej. frenado, giro, cambio de carril) [7]. Los datos del vehículo se recopilan a través de sensores o dispositivos electrónicos, como el Sistema de Posicionamiento Global (GPS, por sus siglas en inglés), acelerómetros, cámaras de video, sensores de distancia del vehículo y el sensor de diagnóstico a bordo (OBDII, por sus siglas en inglés) [91]. Luego, los datos obtenidos se transforman en variables, por ejemplo, velocidad, aceleración, tirón, entre otras; para luego ser procesadas por un modelo computacional el cual determinará automáticamente el estilo de conducción de la persona. Por ejemplo, el trabajo de Brombacher et al., utilizó sensores del módulo inercial y GPS para obtener las variables de desaceleración y aceleración lateral y longitudinal, y determinar si un conductor maneja de forma defensiva o deportiva a través de un ANN [92].

En resumen, a diferencia de la autoevaluación, este enfoque puede ser más costoso debido a los altos precios asociados con el equipo a utilizar (p. ej. vehículo, sensores especializados, GPS) y la recopilación de datos puede resultar difícil de implementar masivamente, puesto que es necesario reclutar conductores que deseen participar en este tipo de estudios. Sin embargo, los resultados son más precisos porque representan estados reales del comportamiento del conductor.

La siguiente sección presenta cómo diferentes modelos automáticos han ayudado a la predicción del estilo de conducción, el tipo de sensores utilizados, así como la recopilación de datos, variables generadas, clase definida, modelos más utilizados y los resultados de predicción de dichos modelos.

\subsection{Clasificación automática del estilo de conducción}

Tal como se mencionó en la sección anterior, los datos del vehículo pueden ser utilizados para implementar modelos de clasificación automática del estilo de conducción. Estos modelos de clasificación pueden dividirse de acuerdo con: 1) la recolección de datos, 2) variables de entrada, 3) etiquetas o clases y 4) los modelos computacionales utilizados en este ámbito. 


\subsubsection{Recolección de datos}

En investigaciones previas, la recolección de datos ha sido realizada ya sea desde un simulador o un vehículo estándar. Un simulador puede estar equipado con un volante, pedales o una palanca de cambios para interactuar con un entorno virtual [16]. Aunque el uso de simuladores puede proporcionar un mejor entendimiento de los estilos de conducción en situaciones de riesgo (p. ej. para estimar la embriaguez o la distracción del conductor), ésta se convierte en una solución costosa, ya que se debe crear un entorno virtual que imite el mundo real. Por otro lado, el uso de un vehículo es cada vez más frecuente, ya que se pueden conectar diferentes dispositivos para registrar automáticamente los datos del vehículo y del conductor. Por ejemplo, un dispositivo GPS puede proporcionar información sobre la posición del automóvil, y obtener la información de velocidad y aceleración mediante cálculos matemáticos [93]. Otro dispositivo popular es el OBDII, que se conecta a un puerto dedicado en el vehículo y puede recopilar datos como la velocidad, el consumo de combustible y la aceleración [13]. Los teléfonos inteligentes también se utilizan con frecuencia para recopilar datos, ya que vienen con sensores más accesibles y confiables, como GPS, cámaras de video de alta definición, acelerómetros y giroscopios [14].

\subsubsection{Variables de entrada}

Dentro de la literatura de trabajos previos se puede reconocer que no existe una combinación perfecta de variables de entrada que permita identificar correctamente los estilos de conducción. Sin embargo, como se observa en la Tabla 3.1, varios estudios revelan que la variable de entrada más utilizada es la aceleración $\left(\mathrm{m} / \mathrm{s}^{2}\right)[12,94]$ y la velocidad $(\mathrm{m} / \mathrm{s})$ [95]. Esta variable generalmente se combina con otras variables como la desaceleración $\left(\mathrm{m} / \mathrm{s}^{2}\right)$ [96] y la dirección del vehículo (rad/s) [97]. Adicionalmente, la variables de aceleración longitudinal y lateral se han utilizado para capturar la forma en que un conductor gira o cambia de carril [15, 92]. Otras variables de entrada que también se consideran para identificar los estilos de conducción son la aceleración y frenado brusco [98, 99]. Estudios previos han considerado la posición del pedal [17], las revoluciones por minuto (RPM) [13] y el tirón [100] para estimar la agresividad. Notoriamente, 
pocos estudios ha considerado a las infracciones de tránsito relacionadas con accidentes de tránsito, como variable de entrada [14, 101, 102].

Tabla 3.1 Variables de entradas extraídas o derivadas del vehículo.

\begin{tabular}{cc}
\hline Variables de entrada & Referencias \\
\hline Velocidad $(\mathrm{m} / \mathrm{s})$ & {$[13,15,17,95,97,103]$} \\
Aceleración $\left(\mathrm{m} / \mathrm{s}^{2}\right)$ & {$[12-14,95,97,103]$} \\
Desaceleración $\left(\mathrm{m} / \mathrm{s}^{2}\right)$ & {$[14,15,92,95,96]$} \\
Aceleración lateral y longitudinal $\left(\mathrm{m} / \mathrm{s}^{2}\right)$ & {$[15,92]$} \\
Aceleraciones y frenados bruscos & {$[98,99]$} \\
Exceso de velocidad $(\mathrm{m} / \mathrm{s})$ & {$[14,101,102]$} \\
Tirón $\left(\mathrm{m} / \mathrm{s}^{3}\right)$ & {$[100]$} \\
\hline
\end{tabular}

En el trabajo propuesto por [101], los autores desarrollaron un sistema para monitorear automáticamente si el conductor cometió infracciones de tránsito mediante la grabación de video en tiempo real. El sistema implementa un módulo de detección, otro módulo de reconocimiento de señales de tráfico y un módulo de registro de infracciones de tráfico, para establecer si el conductor cometió una infracción de tráfico. Además, el sistema aplica técnicas de visión por computadora para identificar las señales de tráfico, las cuales son capturadas por una cámara instalada en la parte superior del vehículo. Cuando la cámara encuentra una señal de tráfico, el sistema hace coincidir la velocidad de la señal de tráfico con la velocidad del vehículo. Luego, si el sistema detecta que el conductor ha cometido una infracción de las señales de tráfico, esto se registra y se presenta en la aplicación de Google Earth.

Otro estudio [102] implementó un sistema que determina las infracciones de "exceso de velocidad" y "no rebasar" a partir de los datos obtenido de los sensores instalados en el vehículo. Para estimar las infracciones de " exceso de velocidad", se colocaron etiquetas RFID en determinadas señales de tráfico en una ruta específica, para comparar la velocidad de las señales de tráfico con la velocidad del vehículo. Además, para calcular la infracción de "no rebasar", se colocaron sensores en el vehículo para determinar si el conductor cruzó la línea de la carretera donde es prohibido adelantar. Además, el sistema incluyó un módulo que envía la infracción de tránsito al Departamento de Policía de Tránsito. 
En los dos estudios mencionados anteriormente se identifican las infracciones de tránsito a través de sensores, pero solo se registra la infracción sin considerar la severidad de esta o si el conductor maneja de una forma segura o insegura tomando en consideración toda la ruta. Un estudio presentado Castignani et al. [14] estima la conducción riesgosa considerando el número de infracciones de tránsito (exceso de velocidad). Este modelo de lógica difusa estima que tan riesgoso puede ser un conductor a través de un puntaje, el cual va disminuyendo si ocurre un evento (por ejemplo, giros bruscos, exceso de velocidad, entre otros). El puntaje tiene valores entre 1 a 100, los valores cercanos a 1 significan que el conductor tuvo una conducción riesgosa y los valores cercanos a 100 que el conductor condujo de manera segura. Para obtener la información de las señales de tránsito lo hizo a través de un servidor web; y con la posición y velocidad de vehículo verificaban si existía exceso de velocidad del conductor.

\subsubsection{Etiquetas o clases}

Las etiquetas o clase se refieren a la discretización del estilo de conducción, ya sea por medio de expertos o por modelos de clusterización, como se explicó en la sección 2.4.1.1. La Tabla 3.2 muestra una síntesis de las categorías de estilos de conducción encontradas en trabajos previos. Como se puede observar en dicha tabla, los estilos de conducción se han categorizado en dos, según los niveles de agresividad, como calma o agresividad [14, 95] y; normal o agresivo [17]. Algunos estudios han establecido una etiqueta adicional, es decir tres categorías, argumentando que los conductores pueden revelar varios niveles de calma o agresividad en una misma ruta. En este sentido, el conductor puede ser etiquetado como tranquilo, moderado o agresivo [7, 16]. Finalmente, algunos estudios informaron el uso de cuatro o más categorías ( $p$. ej. una escala de cinco puntos de muy deportivo a muy defensivo [92] y una escala de cuatro puntos de debajo de lo normal a muy agresivo [104]), pero rara vez se usan, debido a que el número de categorías afecta la exactitud de la predicción del estilo de conducción y la interpretación de los resultados.

Pocos trabajos en este ámbito han detallado el proceso de definición de la etiqueta o clase. Tal como se revisó en el capítulo anterior (sección 2.4.1.1), este proceso es importante para obtener un modelo con un mayor rendimiento. 
Por un lado, investigadores han utilizado la técnica de K-medias para dividir los conductores según corresponda, por ejemplo, calmado y agresivo [17, 18]. Kmedias es una técnica de clusterización, tiene como objetivo dividir las observaciones en 'K' grupos, en los que cada observación pertenece al grupo con la medida más cercana [105]. Sin embargo, esta técnica no es la más recomendable, debido a que puede añadir errores en la generación de las etiquetas, puesto que no existe una validación humana.

Tabla 3.2 Síntesis de las categorías de estilos de conducción utilizados en trabajos previos.

\begin{tabular}{ccc}
\hline No. de categorías & Categorías & Referencias \\
\hline 2 & calmado/ agresivo & {$[14,95,97]$} \\
normal/ agresivo & {$[17,18]$} \\
3 & calmado/ normal/ agresivo & {$[13,15,16,103]$} \\
4 o más & $\begin{array}{c}\text { Riesgo (Bajo, Medio y alto) } \\
\text { Muy Defensivo/ defensivo/ normal } \\
\text { deportivo/ muy deportivo } \\
\text { debajo de normal/normal/ } \\
\text { agresivo/ muy agresivo }\end{array}$ & {$[97]$} \\
& & {$[104]$} \\
\hline
\end{tabular}

Por otro lado, otro trabajo utilizó otra técnica para etiquetar los datos, la cual consiste en primero definir las etiquetas del estilo de conducción para luego crear un conjunto de datos a partir de pruebas de manejo con conductores. A cada conjunto de datos le corresponde una etiqueta establecida, es decir, el conductor ajusta su estilo de conducción dependiendo de la etiqueta. Por ejemplo, se les pide a los conductores que realicen dos o más pruebas de manejo, una de manera agresiva, manejando a alta velocidad, acelerando y frenando de manera brusca; y otra calmada, lo contrario a lo anterior [95]. Esta forma de etiquetar puede resultar poco auténtica, puesto que se obliga a la persona a manejar de una forma en particular, lo cual puede no reflejar la realidad de los comportamientos y hábitos normales de un conductor dependiendo del contexto y ruta donde se encuentre manejando. Similar a este estudio, otro trabajo presentado por Dörr et al., construyó un conjunto de datos sintéticos a través de un programa, uno por cada estilo de conducción (confortable, normal y deportivo) [15]. En este estudio no se explica que criterios utilizaron para concluir los valores de las variables y así poder decidir el estilo de conducción. 
Otra forma de etiquetar los datos que se ha encontrado en la literatura corresponde a la aplicación de autoevaluaciones o cuestionarios en conductores para luego definir el resultado como la etiqueta y utilizar esta etiqueta durante el entrenamiento de un modelo computacional. Por ejemplo, el estudio realizado por Hong et al. describe la aplicación del cuestionario DBQ para luego definir las etiquetas del estilo de conducción en base al puntaje obtenido, es decir, los conductores que tenían puntajes altos eran etiquetados como agresivos y los otros como calmados [99]. Luego, los autores de este trabajo utilizaron un clasificador bayesiano para identificar el estilo de conducción. Uno de los posibles inconvenientes de este procedimiento que las respuestas dadas por los conductores pueden ser subjetivas y pueden no representar el comportamiento real del conductor [89, 90]. Además, este trabajo no explica los rangos del puntaje que se utilizaron para definir el estilo de conducción.

Finalmente, trabajos previos han involucrado a evaluadores que tengan experiencia en el campo de conducción para etiquetar los datos de conductores. Estos evaluadores deben etiquetar al conductor de acuerdo con un conjunto de criterios y luego, a través de una votación, decidir qué etiqueta asignar. Es aconsejable tener un número considerable de evaluadores para medir el grado de acuerdo que existe entre los evaluadores y así evitar cualquier desacuerdo [106]. Por ejemplo, un estudio realizado por Li et al., involucró a tres revisores expertos en el área de conducción, para realizar la evaluación de conductores de acuerdo con el nivel de riesgo (bajo, moderado o alto). Los expertos seleccionaban la etiqueta del conductor dependiendo de las observaciones que hacían a los datos de cada conductor. Para evitar el problema de la subjetividad en este trabajo utilizaron el indicador de Alfa de Cronbach para medir el grado de acuerdo que existió entre los revisores, el cual tuvo un valor de 0.85, obteniendo un buen resultado [107]. Luego, se eligió la etiqueta tenía más votación como la clase de cada instancia. Esta técnica podría ser considerada una de las más confiables para este tipo de problema, sin embargo, a sido poco explorada en el área de estilo de conducción.

\subsubsection{Modelos computacionales}

Se pueden construir diferentes modelos computacionales para clasificar los estilos de conducción. La Tabla 3.3 lista los modelos computacionales, 
configuración y métricas utilizadas para medir la precisión de los estilos de conducción. Según estudios recientes [7, 11], los modelos más utilizados para identificar los estilos de conducción son la lógica difusa, RF, kNN, SVM y ANN. En esta sección se describen los fundamentos teóricos y trabajos previos de los modelos seleccionados.

Tabla 3.3 Modelos computacionales más utilizados para identificar el estilo de conducción, junto con su configuración y métricas para medir su rendimiento.

\begin{tabular}{|c|c|c|c|c|}
\hline \multirow{2}{*}{ Modelo } & \multirow{2}{*}{ Configuración } & \multicolumn{2}{|c|}{ Métricas } & \multirow{2}{*}{ Referencia } \\
\hline & & Exactitud & AUC & \\
\hline $\mathrm{FL}$ & - & 0.90 & & [14] \\
\hline $\mathrm{FL}$ & - & 0.68 & & [15] \\
\hline $\mathrm{FL}$ & 15 reglas & - & & {$[104]$} \\
\hline ANN & $\begin{array}{l}2 \text { capas ocultas } \\
300 \text { neuronas por capa } \\
\text { función tanh }\end{array}$ & 0.90 & & [103] \\
\hline ANN & $\begin{array}{l}1 \text { capa oculta } \\
9 \text { neuronas }\end{array}$ & 0.77 & & [13] \\
\hline ANN & $\begin{array}{l}\text { No utilizo capas intermedias } \\
\text { Modifico los pesos de los nodos de entrada }\end{array}$ & 0.83 & & [95] \\
\hline SVM & $\begin{array}{l}\text { Función de base radial } \\
\text { No reporto parámetros }\end{array}$ & 0.60 & & [96] \\
\hline SVM & Kernel polinomial & 0.87 & & [108] \\
\hline SVM & Kernel lineal & 0.86 & & [17] \\
\hline $\mathrm{RF}$ & $\begin{array}{l}n \text {-arboles }=200 \\
n \text {-capas } 10\end{array}$ & & 0.99 & [10] \\
\hline $\mathrm{RF}$ & $\mathrm{n}$-arboles $=40$ & 0.69 & & [109] \\
\hline kNN & $\mathrm{k}=5$ & 0.68 & & [109] \\
\hline kNN & - & 0.98 & & [110] \\
\hline
\end{tabular}

\subsubsection{Lógica difusa}

La lógica difusa se ha utilizado para clasificar diferentes estilos de conducción (p. ej. tranquilo o agresivo [8, 104]; cómodo, normal y deportivo [15]) debido a su simplicidad de implementación e interpretación de resultados. Por ejemplo, en el trabajo publicado por Castignani et al., [14] se clasificó a 10 conductores como calmados o agresivos utilizando aceleración, frenado, dirección y exceso de velocidad como variables de entrada del modelo de lógica difusa. En su modelo de lógica difusa, los autores utilizaron diferentes eventos del conductor y factores externos, como el clima y la hora del día, y reportaron 
una precisión del modelo de 0.90. En otro estudio publicado por Dörr et al., [15] obtuvo una precisión de 0.68 al tratar de identificar los estilos de conducción como cómodo, normal o deportivo utilizando variables de entrada provenientes de datos simulados, como la velocidad, la desaceleración, la aceleración y el intervalo de tiempo. A diferencia del estudio anterior, Aljaafreh et al., [104] diseñó y probó un modelo utilizando datos reales y el asesoramiento de tres expertos. El modelo se implementó utilizando tres variables de entrada que son: velocidad, aceleración lateral y aceleración longitudinal. Estas variables de entrada se utilizaron para diseñar las quince reglas del sistema de lógica difusa, y la salida del sistema estima estilos de conducción normales o agresivos. Este estudio no informó ninguna métrica de rendimiento del modelo de lógica difusa. Aunque estos ejemplos proporcionan una idea general sobre el diseño e implementación de modelos de lógica difusa, no hay consenso sobre su efectividad, ya que estos estudios han reportado métricas de clasificación diferentes o nulas, como se puede observar en la Tabla 3.3. Además, algunos de estos estudios no presentan las reglas de modelo difuso, lo que dificulta su reproducibilidad.

\subsubsection{Red neuronal artificial}

En el área de identificación del estilo de conducción, varios estudios han implementado modelos ANN, reportando diferentes valores de exactitud y varias configuraciones (p. ej., número de capas y función de activación). La Tabla 3.3 muestra varios trabajos realizados en este ámbito. Por ejemplo, el modelo ANN presentado por Cheng et al. [16] se configuró con dos capas ocultas, 300 neuronas por capa, y la función de activación de tanh. Las variables de entrada que alimentan el modelo de ANN fueron la aceleración, ángulo del pedal y velocidad. Este modelo ANN dio una exactitud de 0.90. Por otro lado, el trabajo presentado por Meseguer et al. [13], en donde realizaron pruebas de manejo reales, implementó un ANN con una capa oculta y nueve neuronas. Este modelo tenía como objetivo clasificar los estilos de conducción como agresivos, normales o tranquilos utilizando datos de velocidad, aceleración y RPM como variables de entrada. Los autores informaron una precisión de 0.77 . Otro trabajo relacionado presentado por Jiménez et al. [95] implementó un modelo ANN para identificar estilos de conducción como tranquilos o agresivos utilizando el consumo de energía del vehículo como variable de entrada. A diferencia de los 
dos estudios anteriores, la arquitectura propuesta para la red neuronal tiene una red perceptrónica, que cambia solo los pesos de la neurona y usa datos de velocidad, aceleración, desaceleración y pedal del acelerador como variables de entrada. Este modelo ANN logró una exactitud de 0.83. En resumen, tal como se muestra en la Tabla 3.3, estos estudios proporcionan una visión general de cómo se han implementado los modelos ANN para la clasificación de los estilos de conducción. Sin embargo, estos estudios no presentan un enfoque sistemático para ajustar los parámetros necesarios y configurar el modelo ANN, dificultando la reproducibilidad de los experimentos.

\subsubsection{Máquina de soporte vectorial}

Debido a su idoneidad para trabajar con problemas del mundo real, este modelo ha sido últimamente utilizado en el reconocimiento de estilos de conducción. Estudios previos han reportado diferentes configuraciones (p. ej., función kernel, valores gamma), variables de entradas y la evaluación del modelo mediante valores de exactitud. La Tabla 3.3 muestra varios trabajos en donde se han implementado modelos de SVM. Por ejemplo, el trabajo publicado por Van Ly et al. [96] reportó la implementación un modelo SVM para identificar a los conductores mediante el modelado de eventos del conductor (es decir, maniobras), como frenado, aceleración y giro utilizando variables de entrada como aceleración, desaceleración y velocidad angular. El modelo se configuró a través de la función kernel de Base Radial Gaussiana (RBF, por sus siglas en inglés), con una precisión de 0,60. Otro estudio propuesto por Zhang et al. [108] implementó un modelo SVM usando una función kernel polinomial; y la aceleración, posición del acelerador y datos de RPM del motor como variables de entrada obteniendo una exactitud del 0.87 . Por otro lado, el estudio presentado por Wang et al. [17] implementó dos modelos SVM para clasificar los estilos de conducción normales y agresivos a partir de los datos de velocidad y aceleración. El primer modelo se implementó usando un kernel RBF y el segundo con un kernel lineal, con una precisión de 0.77 y 0.86 , respectivamente. En resumen, estos estudios muestran varias configuraciones de parámetros para el modelo SVM, con valores aceptables de exactitud para la identificación del estilo de conducción. Sin embargo, como se mencionó anterior mente, hace falta 
evidencia sobre cómo ajustar los parámetros necesarios para mejorar el rendimiento del modelo.

\subsubsection{Bosques Aleatorios}

Los bosques aleatorios (RF) también han sido entrenados para tareas de clasificación de estilo de conducción. Por ejemplo, Júnior et al. [10] probó diferentes modelos de clasificación para identificar eventos de conducción agresivos y no agresivos a partir de datos recopilados con un teléfono inteligente. Los autores informaron que el modelo de RF ( $n$-árboles $=200 ;$ m-capas $=10$ ) superó a los otros modelos al alcanzar un valor de Área Bajo la Curva (AUC, por sus siglas en inglés) de 0.99. Por el contrario, Karginova et al. [109] comparó varios clasificadores obteniendo resultados no tan buenos. El modelo RF ( $\mathrm{n}$-trees =40), tuvo una precisión general de 0.685. Los conductores fueron clasificados en normales o bruscos, las variables usadas fueron la velocidad, el tirón, la tasa de combustible, entre otras; y fueron recopiladas utilizando un dispositivo OBDII. En estos trabajos se puede observar la utilización de diferentes métricas, como exactitud y AUC, configuraciones de nodos (220 y 40); y dispositivos electrónicos (OBDIl y teléfonos inteligentes).

\subsubsection{5 k-vecinos más cercanos}

Un estudio realizado por Vaitkus et al. [110], reportó la implementación de un modelo kNN analizando los datos de aceleración longitudinal y vertical de un sensor de unidad de medida inercial (IMU). Los autores no informaron el número de $k$, y el modelo obtuvo una precisión de 0,98. Por otro lado, Karginova et al. [109] comparo varios clasificadores, entre ellos el kNN, obteniendo un bajo rendimiento. El modelo $\mathrm{kNN}(\mathrm{k}=5)$ alcanzo una precisión de 0.68 . De estos dos estudios se puede observar la carencia de rigurosidad al momento de reportar y evaluar estos modelos, puesto que, no se reporta toda la información necesaria (p. ej. k en [110]) para replicar el experimento.

\subsubsection{Modelos estadísticos}

Existen otros métodos para identificar el estilo de conducción, como los modelos estadísticos, que usan la probabilidad para agrupar un conjunto de datos semejantes. El trabajo de Eren et al. [111], utilizó redes bayesianas para determinar si un conductor maneja de manera riesgosa o segura. Este trabajo 
reportó la captura de 15 pruebas de manejo para entrenar el modelo, el cual obtuvo 0.933 de precisión. Otro estudio utilizó el modelo oculto de Márkov para identificar eventos de conducción, como girar a la izquierda, derecha y cambio de carril; obteniendo una precisión de 0.913 [112]. Por otro lado, el trabajo propuesto por Koh y Kang [113], reportó un sistema para clasificar las conductas agresivas en 15 conductores a través de un teléfono inteligente. Los autores implementaron un modelo de mezcla gaussiana utilizando la aceleración lateral como variable de entrada para detectar el perfil de agresividad de los conductores. Dicho trabajo no reporto resultados. Aunque en la literatura se encuentran estos modelos estadísticos, no son tan utilizados en este contexto.

\subsection{Limitaciones y desafíos}

Este capítulo abordó los fundamentos teóricos y trabajos recientes en el área de estilos de conducción, quedando de manifiesto que para la correcta identificación automática de estilos de conducción se necesita la selección de 1) sensores o dispositivos que permitan la captura de los datos del vehículo; 2) variables de entrada primarias o derivadas de los datos del vehículo; 3) etiquetas o clase y 4) la selección de modelos computacionales.

Tal como se describió en este capítulo, existen un sinnúmero de modelos computacionales que han sido implementados para identificar estilos de conducción. Sin embargo, en los estudios revisados se encuentran que existen varias limitaciones al momento de implementar un modelo de clasificación del estilo de conducción.

Una limitación es que cada estudio aplica diversos criterios para la configuración de los parámetros de cada modelo, algunos realizando una búsqueda profunda, otros aplicando una búsqueda aleatoria, y más aún, otros trabajos no reportan qué tipo de configuración utilizaron en su modelo. En este sentido, existe una falta de evidencia sobre la optimización de los parámetros de cada modelo. Debido a la falta de sistematización en cuanto a la optimización de los parámetros de los modelos computacionales, no se puede justificar el mejor modelo computacional para realizar la identificación automática de los estilos de conducción. 
Otra limitación encontrada es que pocos estudios explican cómo se etiquetaron los datos para entrenar y validar los modelos. Como se revisó en la sección 3.4.3, existen diversas categorías que pueden determinar el estilo de conducción. Es usual encontrar en este tipo de trabajos varias formas de determinar dicha categoría, como, por ejemplo, a través de algoritmos de clusterización, utilizando un cuestionario de autoevaluación o por medio de evaluadores expertos. Los trabajos revisados en este capítulo no explican concretamente cómo se realizó la categorización del estilo del conductor. Por lo tanto, existe una falta de criterio al momento de establecer la categoría del conductor.

Por otro lado, los estudios presentados en este capítulo demuestran la complejidad de identificar automáticamente el estilo de conducción, puesto que la mayoría de éstos entrenan sus modelos computacionales con pocos datos. Tal como se explicó en la sección 3.3.2, utilizar datos del vehículo puede resultar costoso, debido al equipo especializado necesario y también a la ardua tarea de reclutar conductores que se encuentren interesados en participar en estos estudios. Este problema es muy habitual cuando se trata con datos reales, por lo que es necesario proveer de mecanismos que permitan mejorar la predicción del estilo de conducción y que se ajusten a una muestra limitada.

Otro desafío encontrado en la revisión de trabajos previos es falta de consentimiento con respecto a la forma de evaluación de los modelos de predicción. Como se presentó en la sección 3.4.4 la exactitud y el AUC son las métricas que se han utilizado en estudios pasados para evaluar el rendimiento de los modelos computacionales. Sin embargo, de acuerdo con la literatura de aprendizaje automático se pueden utilizar otras métricas y métodos de evaluación, dependiendo del conjunto de datos que se tiene (p. ej. datos balanceados vs. datos no balanceados). Por lo tanto, es necesario emplear una evaluación integral que permita transparentar el rendimiento de dichos modelos computacionales.

Finalmente, como se abordó en la Sección 3.4.2, existen pocos estudios que utilizan las infracciones de tráfico como variable de entrada. Esto se debe a que la determinación de información confiable sobre las infracciones de tráfico 
puede ser una tarea complicada para los departamentos de Tránsito. Si bien los cuestionarios y la información estadística brindan información poco fundada debido a la subjetividad de los datos de la percepción del conductor y los datos incompletos de los informes de la policía de tránsito, el reconocimiento automático de las infracciones del conductor podría detectar y estimar las infracciones de las señales de tránsito de una forma más confiable, tomando en cuenta los datos del vehículo y las señales de tránsito existentes en una ruta. Dentro de los trabajos presentados para este fin, se propone el uso de equipamiento costoso como cámaras, o sensores especializados ubicados en el vehículo o en las señales de tránsito de una ruta específica. Sin embargo, no existe una solución eficiente que permita aprovechar los datos del vehículo para inferir las infracciones de tráfico.

\subsection{Resumen}

En resumen, si bien los estudios previos presentados en esta sección han logrado identificar el estilo de conducción hasta cierto punto, estos modelos implementados no siguen una metodología sistematizada, lo que impide la replicación de experimentos y la adaptación de estos modelos propuestos en otro contexto. Como se explicará en los siguientes capítulos, este trabajo intenta mejorar las limitaciones antes mencionadas: 1) proponiendo una metodología sistematizada, tomando en cuenta la teoría de aprendizaje automático y las buenas prácticas para la creación de modelos computacionales como ha sido presentado en el Capítulo 2, y 2) diseñando e implementando un modelo automático que permita inferir las infracciones de tránsito que un conductor realiza durante una ruta específica. 


\section{Capítulo 4: Clasificación automática de estilos de conducción}

En este capítulo se detallará el diseño de la metodología propuesta y su aplicación a través de un experimento. Se explicará cada etapa de la metodología propuesta, con sus respectivas tareas, para la implementación de los modelos computacionales. Se describirá como se va a recolectar los datos, las variables de entrada seleccionada, como se realizar la construcción de cada modelo y finalmente la elección de las métricas de evaluación de rendimiento.

\subsection{Metodología para la clasificación automática de estilos de conducción}

Tal como se expresó en el capítulo anterior, existe la necesidad de una metodología sistemática para la clasificación automática de los estilos de conducción. Este trabajo propone una metodología para diseñar, implementar y evaluar modelos computacionales que detecten el estilo de conducción, basándose en propuestas y guías sobre el proceso de implementación de modelos computacionales [20-22], así como en las limitantes que se discutieron en la sección 3.5. Como se observa en la Figura 4.1 esta metodología consta de cuatro etapas: 1) recopilación de datos; 2) generación de características; 3) construcción del modelo; y 4) evaluación del modelo, las cuales se adaptan a los objetivos planteados en este trabajo (ver sección 1.2).

En la etapa de recopilación de datos se definirán los participantes (edad, género, experiencia) que realizarán la prueba de manejo, la ruta específica con sus características (señales de tránsito, duración, distancia, tipos de vía, entre otros), y los dispositivos que serán utilizados para capturar los datos del vehículo, posición del vehículo, ubicación de las señales de tránsito, etc. Luego que se han recopilado los datos de una muestra de conductores, se procederá a generar las variables de entrada, necesarias para entrenar el modelo. Existen muchas variables que pueden ser derivadas de los dispositivos usados y datos capturados, como, por ejemplo, de la velocidad se puede calcular la aceleración, la cual serviría como variable de entrada. Una vez que las variables de entrada 
han sido definidas, se llevará a cabo la construcción del modelo, el cual cuenta con varias tareas como: a) la definición de etiquetas, b) la generación de datos sintéticos, y la configuración de los hiperparámetros. Para la definición de etiquetas, primero hay que considerar cuáles etiquetas son relevantes para este problema, para posteriormente realizar una evaluación manual involucrando a expertos en el área. Debido a la dificultad de conseguir participantes, se puede considerar la generación de datos sintéticos para mejorar el proceso de entrenamiento de los modelos. La configuración de los hiperparámetros se realizará una vez que se definan los modelos que se entrenarán. Finalmente, se verifica el rendimiento de los modelos computacionales en la etapa de evaluación del modelo, la cual consta de dos evaluaciones: a) el cálculo de métricas de la matriz de confusión, dependiendo de las características del conjunto de datos que se tenga; y b) pruebas de significancia estadística. La aplicación de estas dos evaluaciones permitirá discutir y decidir el o los mejores modelos computacionales para clasificar automáticamente el estilo de conducción.

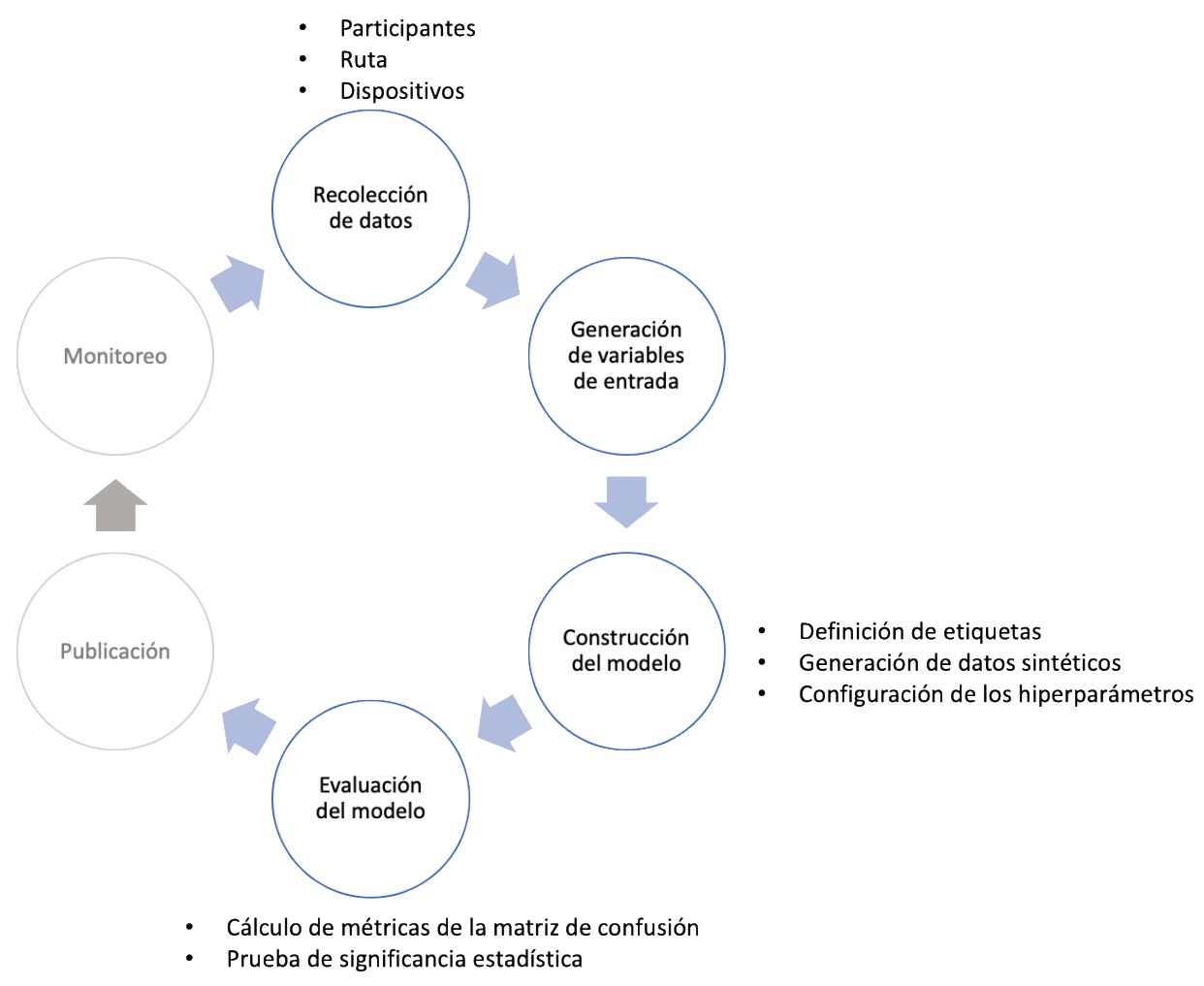

Figura 4.1 Metodología propuesta para la clasificación automática de estilos de conducción. 
No se han considerado las etapas de publicación y monitoreo de los modelos, puesto que estas dos no son parte del alcance del estudio. De ser el caso, se puede obtener más información sobre estas dos etapas en estos trabajos [20, 21].

\subsection{Experimento ilustrativo de la metodología propuesta}

Para ilustrar esta metodología, se realizó un experimento aplicando las cuatro etapas. A continuación, se detallan cada una de estas etapas.

\subsubsection{Recolección de datos}

Esta subsección detalla la información de cómo se realizaron las pruebas de manejo para la captura de los datos, la información de los participantes, las rutas seleccionadas y el equipo utilizado para recopilar datos durante las pruebas de manejo.

\subsubsection{Participantes}

Un total de 50 conductores (31 hombres, 19 mujeres, promedio $=48.24$ años, dev. std. = 10.61 años) participaron en las pruebas de manejo. Los conductores tenían una experiencia de manejo promedio de 15.78 años (dev. std. = 10.01). En términos de edad, 22 conductores tenían entre 25 y 34 años, 19 conductores entre 35 y 54 años y 9 conductores entre 55 y 65 años. Todos los conductores contaban con una licencia de conducir no profesional.

\subsubsection{Ruta}

Se seleccionaron dos rutas similares, una en la ciudad de Guayaquil (se realizaron 40 pruebas de manejo) y otra en Madrid (se realizaron 10 pruebas de manejo). Se eligió una ruta en la ciudad de Guayaquil-Ecuador en la que se presentan diversas señales de tránsito como: pare, velocidad máxima, zona de seguridad escolar, paso de peatones, señales de semáforo, entre otras. A través de la ruta seleccionada, se identificaron múltiples zonas urbanas, zonas residenciales y avenidas, asociadas con sus límites de velocidad. Estos límites de velocidad pueden variar desde $20 \mathrm{~km} / \mathrm{h}$ hasta $90 \mathrm{~km} / \mathrm{h}$. La Figura 4.2 muestra la ruta seleccionada de este experimento para recopilar los datos del conductor. El recorrido tiene una duración aproximada de 25 minutos, empieza en el "km. 2.5 vía Samborondón", pasando por el puente de la "Unidad Nacional", luego por 
el centro de Guayaquil y regresando al punto de partida. En la siguiente subsección presentamos cómo recopilamos los datos para implementar el modelo de lógica difusa.

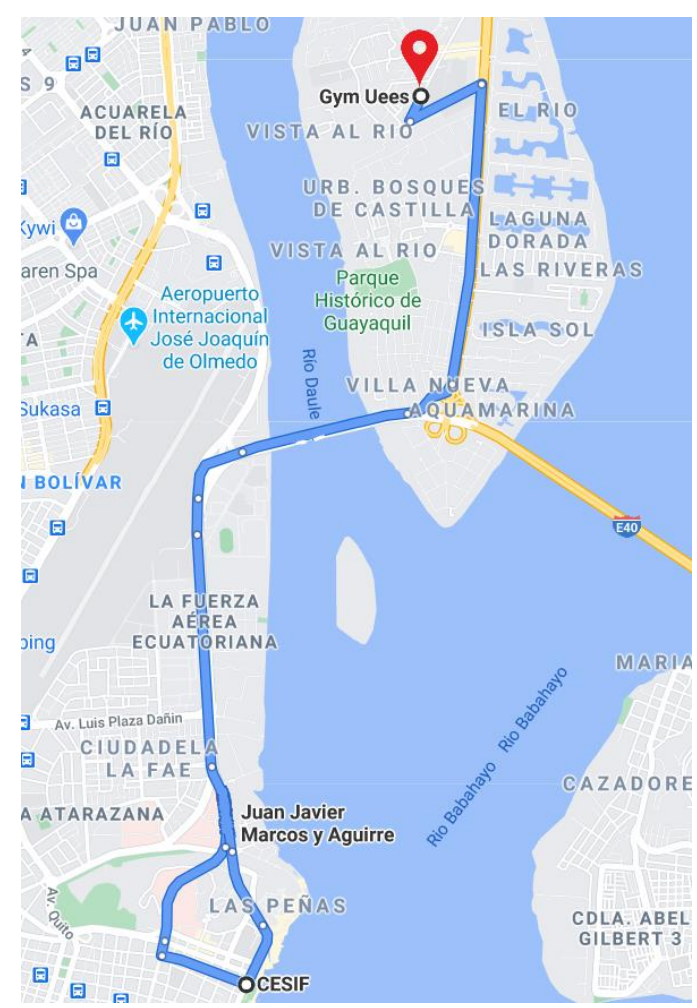

Figura 4.2 Ruta seleccionada para la ciudad de Guayaquil.

La ruta de la ciudad de Madrid tiene una duración de 24 minutos y una distancia de 18 kilómetros. Como se muestra en la Figura 4.3 el recorrido de la ruta empieza en la Av. Campos Sur (INSIA), luego pasa por la A3 hasta llegar atocha para regresar hasta el punto de inicio. Esta ruta tuvo una duración de aproximadamente 23 minutos y una distancia promedio de 18 kilómetros. Además, tiene 23 señales de límite de velocidad (por ejemplo, 40 km, 70 km, 90 $\mathrm{km}$ ) e incluían diferentes tipos de ruta (por ejemplo, autopistas, carreteras suburbanas y carreteras urbanas). 


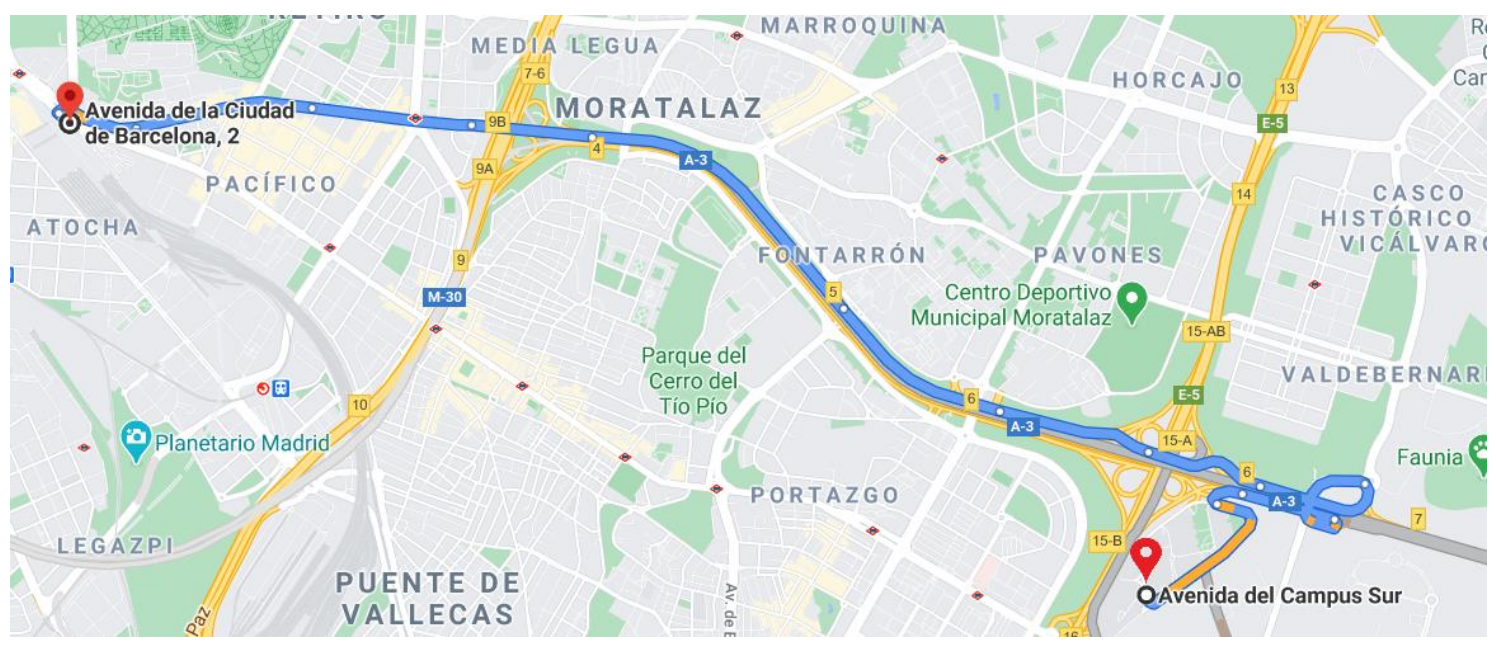

Figura 4.3 Ruta seleccionada para la ciudad de Madrid.

\subsubsection{Dispositivos}

Se usaron dos dispositivos para recopilar datos en el vehículo: un dispositivo OBDII, conectado al automóvil y un teléfono inteligente con la aplicación Torque instalada para recibir datos OBDII a través de Bluetooth. El OBDII capturó y envió los datos de velocidad del vehículo a una frecuencia de muestreo de $1 \mathrm{~Hz}$. La aplicación Torque [114] guardó los datos de posición GPS (latitud y longitud) del teléfono inteligente y la marca de tiempo para cada registro. Por lo tanto, se infiere que la posición del teléfono inteligente es la posición del vehículo.

Para guardar la información de las señales de tránsito, se registró la posición GPS de las señales de tráfico con un teléfono inteligente y la información de esta (por ejemplo, $50 \mathrm{~km} / \mathrm{h}$ ). Como este paso se realizó manualmente, prevemos que para capturar datos automáticamente se necesitaría sensores adicionales (por ejemplo, cámaras de video para extraer señales de tráfico, acceder a un servicio web), lo cual se podría realizar en un trabajo futuro.

\subsubsection{Prueba de manejo}

Los conductores realizaron la prueba de manejo en un vehículo pequeño o mediano, dependiendo de la disponibilidad. Todas las pruebas se realizaron durante la mañana o la tarde, en un día despejado y con diferente flujo de tráfico. Antes de comenzar las pruebas de manejo, se les pidió a los participantes que condujeran el vehículo durante un período corto para acostumbrarse al vehículo (por ejemplo, cambiar la velocidad). Un investigador estuvo presente como 
copiloto durante las pruebas de manejo para dar instrucciones a los conductores sobre la ruta a seguir.

\subsubsection{Variables de entrada}

El criterio para la selección de las variables de entrada para este estudio depende de las variables más utilizadas en trabajos previos (Sección 3.4.2) y que estén relacionadas con la seguridad vial.

La aceleración $\left(\mathrm{m} / \mathrm{s}^{2}\right)$ es la característica más utilizada para clasificar el estilo de conducción. Esta característica se calculó a partir de los datos de velocidad $(\mathrm{km} / \mathrm{h})$ del vehículo obtenidos del OBD2. Cuatro variables de entrada se derivaron de la aceleración. Las dos primeras variables de entrada fueron la aceleración (AccPro) y la desaceleración promedio (DecPro) de toda la ruta. La tercera y cuarta variable corresponden a la ocurrencia total de eventos de aceleración brusca (EveAcc) y frenado brusco (EveFre) durante la ruta. Estas dos características se calcularon de acuerdo a un conjunto de umbrales de la aceleración instantánea, como se informa en un estudio previo [115].

Considerando que los conductores no exhiben el mismo comportamiento en una ruta congestionada que en una ruta libre, se establecieron tres niveles de flujo de tráfico para derivar estas características: bajo, medio y alto. Como se muestra en la Tabla 4.1, se seleccionaron diferentes valores de umbral para los tres niveles de flujo de tráfico. Por lo tanto, para contar el número total de EveAcc o EveFre, primero se estableció el nivel de tráfico de la ruta (es decir, bajo, medio, alto), que fue definido por el investigador que estuvo presente durante las pruebas de manejo. Luego, se contó cuántas veces la aceleración instantánea tuvo un valor igual o mayor que su umbral correspondiente para EveAcc y EveFre.

Por ejemplo, si el conductor A realizó la prueba de manejo en una ruta traficada, el investigador asignó un alto nivel de flujo de tráfico. Con los datos recopilados del conductor $A$, se calculó la aceleración instantánea y contamos las veces que este valor era igual o mayor que su umbral correspondiente de 1 $\mathrm{m} / \mathrm{s}^{2}$. Este valor correspondió a la ocurrencia total de eventos de aceleración brusca. Del mismo modo, se contó las veces que la aceleración instantánea fue 
igual o inferior a su umbral de $-1 \mathrm{~m} / \mathrm{s}^{2}$. Este valor corresponde a la ocurrencia total de eventos de frenado brusco.

Tabla 4.1 Umbrales definidos para eventos de aceleración brusca y de frenado brusco dependiendo del nivel de flujo de tráfico.

\begin{tabular}{cccc}
\hline \multirow{2}{*}{ Tipo de Eventos } & \multicolumn{3}{c}{ Niveles de flujo de tráfico } \\
\cline { 2 - 4 } EveAcc & Bajo & Medio & Alto \\
\cline { 2 - 4 } EveFre & $a<=2 \mathrm{~m} / \mathrm{s}^{2}$ & $a>=1.5 \mathrm{~m} / \mathrm{s}^{2}$ & $a>=1 \mathrm{~m} / \mathrm{s}^{2}$ \\
& $a<=2 \mathrm{~m} / \mathrm{s}^{2}$ & $a<=1.5 \mathrm{~m} / \mathrm{s}^{2}$ & $a<=1 \mathrm{~m} / \mathrm{s}^{2}$ \\
\hline
\end{tabular}

Finalmente, la quinta variable de entrada, las infracciones de tráfico (InfracTra), se derivó de los datos recopilados de cada conductor. Para obtener las infracciones de tráfico, se modeló un sistema de lógica difusa a partir de los datos del vehículo. El modelo tiene dos entradas: 1) el exceso de velocidad del conductor, que se calcula a partir de la diferencia entre el límite de velocidad y la velocidad del vehículo cuando está cerca de una señal de tráfico; y 2) la gravedad de la infracción de tránsito, que está determinada por expertos y leyes de límite de velocidad. La salida del modelo es un valor entre 0 y 1 , donde un valor de 0 significa que un conductor tuvo un nivel bajo de infracciones de tráfico, y un valor de 1 significa que un conductor tuvo un alto nivel de infracciones de tráfico. A continuación, se describirá como se calculó la variable InfracTra a través de un experimento.

Tal como se mencionó en las limitaciones y desafíos de trabajos previos (sección 3.5), hay una falta de estudios que determinen que tan seguro o inseguro maneja un conductor considerando las infracciones de tránsito. Por tal motivo, este trabajo propone un modelo computacional para estimar el comportamiento de conducción segura considerando las infracciones de tráfico, que servirá como una variable de entrada en la metodología propuesta para la clasificación del estilo de conducción. Esta sección describe el experimento que se realizó para diseñar e implementar dicho modelo computacional.

El objetivo de este experimento es estimar el comportamiento de conducción segura basado en las infracciones de tráfico. Por lo tanto, para estimar el comportamiento de conducción segura, se propone un modelo de lógica difusa, debido, su capacidad para modelar el razonamiento humano. El diseño de este modelo se basa en que, dependiendo de la gravedad de las infracciones de tráfico, estas pueden provocar accidentes de tránsito. Por 
ejemplo, es más probable que ocurra un accidente si un conductor irrespeta una señal de pare en lugar de ir a una de velocidad de $55 \mathrm{~km} / \mathrm{h}$ en una zona de 50 $\mathrm{km} / \mathrm{h}$.

En la Figura 4.4 se muestra una descripción general de la arquitectura del modelo propuesto. Primero, se recopila los datos del vehículo, de las señales de tráfico y del teléfono inteligente (posición GPS). Luego, se lleva a cabo el procesamiento de datos para calcular la distancia más cercana entre el automóvil y la señal de tránsito, para determinar si existió exceso de velocidad. Los últimos componentes corresponden al modelo de lógica difusa, el cual cuenta con variables de entrada, exceso de velocidad y severidad de la infracción de tráfico, procesamiento del modelo de lógica difusa y finalmente la salida. En secciones posteriores se explicará en detalle cómo se implementó el modelo de lógica difusa.

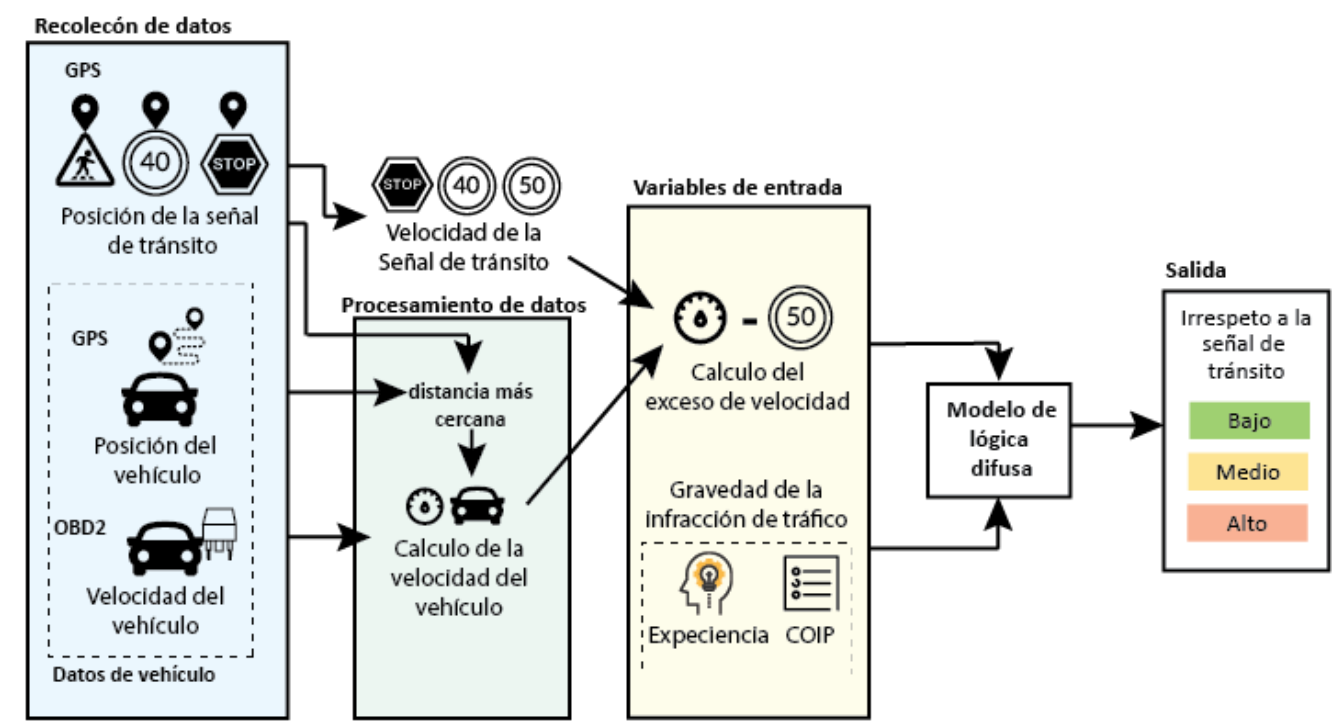

Figura 4.4 Arquitectura del modelo para estimar el comportamiento de conducción segura a través de las infracciones de tránsito

\subsubsection{Participantes y ruta}

Se pidió a quince personas (9 mujeres, 6 hombres), de 22 a 63 años, con licencia de conducir no profesional para participar en este experimento. La experiencia de los conductores varía entre 5 a 45 años. Las pruebas se realizaron en vehículos pequeños o medianos y solo el instructor estuvo de acompañante para dar información sobre la ruta. La ruta se realizó en la ciudad de Guayaquil y es la misma presentada en la sección 4.2.1.2. 


\subsubsection{Recolección de datos}

Este experimento recopila datos de la ubicación de las señales de tránsito y los datos del automóvil para estimar el comportamiento de conducción segura. A continuación, se explica cómo recopilamos los datos y qué equipos se utilizaron.

a) Posición de la señal de tráfico: como se menciona anteriormente, se necesita saber la posición de cada señal de tránsito de la ruta seleccionada. Por lo tanto, se utilizó la aplicación "GPS Latitude Longitude" que se ejecuta en un teléfono inteligente con el sistema operativo Android, para guardar la latitud y longitud de cada señal de tránsito. Como resultado, se detectaron 23 señales de tránsito en la ruta con sus respectivos límites de velocidad, (es decir, 90, 70, 50, $45,40,20 \mathrm{~km} / \mathrm{h}$ y pare).

b) Datos del vehículo: se utilizó el dispositivo de diagnóstico a bordo (OBD2), conectado al vehículo, que envía los datos a un teléfono inteligente a través de bluetooth. Luego, se instaló la aplicación "Torque", para visualizar y guardar los datos del vehículo de las pruebas de manejo. Torque guarda la velocidad, la posición del teléfono inteligente (del GPS incorporado) y una marca de tiempo durante la prueba del conductor.

\subsubsection{Generación de características}

El siguiente paso es obtener la velocidad del vehículo con respecto a la proximidad con la señal de tráfico. La detección del exceso de velocidad del conductor se calcula a partir de la posición del vehículo y la ubicación de la señal de tránsito. Para ello, se utilizó la fórmula de Haversine, para medir la distancia entre dos puntos de sus latitudes y longitudes [116]. Por lo tanto, el primer punto es la posición GPS de la aplicación Torque (posición del vehículo), mientras que el segundo punto es la posición GPS de la señal de tránsito guardada en la aplicación GPS Latitude Longitude. Para comprobar si hubo o no infracción de tránsito, se elegio la distancia más cercana de ambas posiciones, para luego obtener la velocidad del vehículo y compararla con el límite de velocidad de la señal de tránsito. 


\subsubsection{Modelo de lógica difusa}

La estimación del nivel de infracciones de tránsito se basa en evidencia empírica, establecida por la experiencia humana, el comportamiento del conductor durante la recolección de datos y las leyes definidas por instituciones gubernamentales como el Código Orgánico Integral Penal (COIP) [117]. El modelo de lógica difusa se implementó utilizando la herramienta MATLAB. Específicamente, se usó el controlador Sugeno, porque el proceso necesita defuzzificación. Además, el modelo utiliza funciones triangulares y trapezoidales. Como resultado del modelo, a cada conductor se le asigna un valor que representa el nivel de infracciones de tránsito. Para entrenar el sistema difuso, se realizaron experimentos con datos simulados y para validara el modelo se utilizaron los datos reales que se obtienen en pruebas de manejo. Para la creación de los datos simulados se recogieron una serie de casos. La elección de valores se basa en la simulación de situaciones poco seguras, inseguras, seguras, etc., por un usuario experto.

a) Variables de entrada. se definieron dos variables: exceso de velocidad, que es la diferencia entre la velocidad de la señal de tránsito y la del vehículo y; la severidad de la infracción de tránsito, las etiquetas de esta entrada se definieron tomando en consideración el COIP.

b) Salida. la estimación de la infracción de tráfico tiene tres valores posibles: bajo, medio y alto. Como se mencionó anteriormente, la gravedad de la infracción de tráfico juega un papel importante para construir el modelo difuso, ya que no tiene el mismo impacto que el conductor irrespete una 'señal de pare' que el exceso de velocidad entre $5 \circ 10 \mathrm{~km} / \mathrm{h}$ en un camino recto. Por lo tanto, se consideró una infracción de tráfico alto cuando la infracción es severa y una infracción de tráfico bajo cuando la infracción es leve o no se irrespeto la señal de tráfico. Así, la variable de infracción de tráfico tiene un valor en el intervalo $[0,1]$, con un valor 0 para una infracción de tráfico baja (leve) y 1 para una infracción de tráfico alta (grave).

c) Funciones de pertenencia y etiquetas lingüísticas. cada variable de entrada tiene su propia función de pertenencia con sus respectivos valores como se muestra en la Figura 4.5. La variable de entrada de exceso de velocidad se define en un rango de 0 a $25 \mathrm{~km} / \mathrm{h}$ y, en consecuencia, se divide en tres intervalos 
(etiquetas lingüísticas): bajo, medio y alto, cada uno con su función de pertenencia y valores. La variable de severidad se define en un rango de 0 a 1 y con tres etiquetas lingüísticas: baja, moderada y grave, cada una con sus propias funciones de pertenencia y valores.

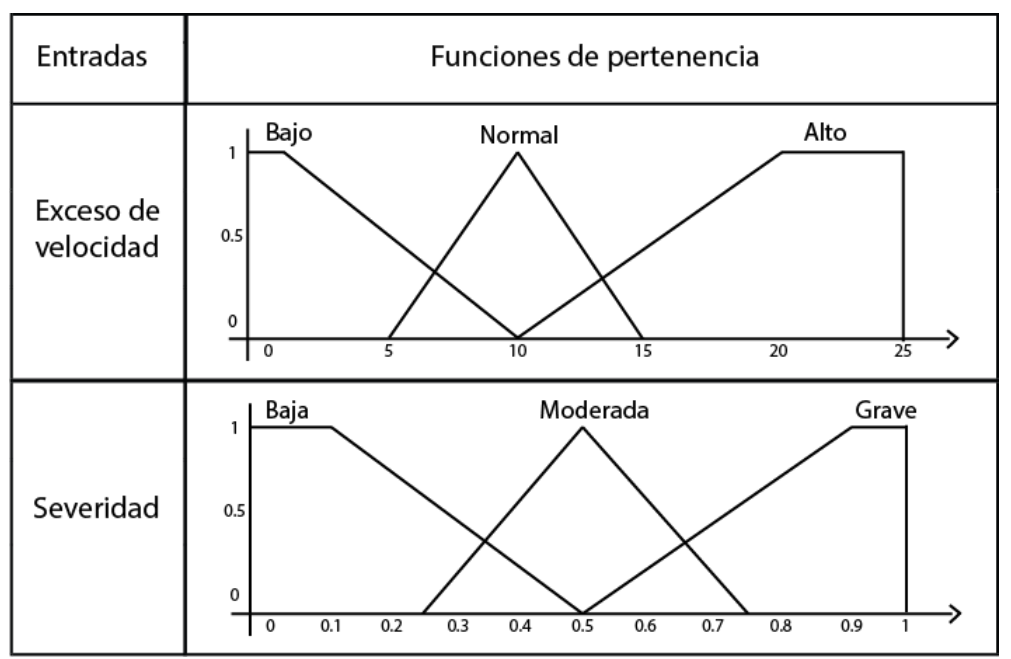

Figura 4.5 Descripción de las variables de entrada con sus etiquetas lingüísticas y funciones de pertenencia.

d) Reglas: Asignamos una infracción de tráfico alta a los casos en los que la etiqueta de gravedad es severa y con un exceso de velocidad superior a 15 $\mathrm{km} / \mathrm{h}$. Además, consideramos una infracción de tráfico bajo cuando el exceso de velocidad es bajo. Según estas premisas, la Tabla 4.2 presenta las reglas establecidas:

Tabla 4.2 Reglas definidas para las variables de entrada.

\begin{tabular}{ccccc}
\hline Reglas & \multicolumn{2}{c}{ Antecedentes } & $\begin{array}{c}\text { Consecuent } \\
\text { e }\end{array}$ & Peso \\
\hline 1 & $\begin{array}{l}\text { Exceso de } \\
\text { velocidad } \\
\text { Exceso de } \\
\text { velocidad }\end{array}$ & Alto & Alta & 1 \\
& $\begin{array}{c}\text { Alto } \\
\text { Beveridad }\end{array}$ & Media & 1 \\
3 & $\begin{array}{l}\text { Exceso de } \\
\text { velocidad } \\
\text { Severidad }\end{array}$ & $\begin{array}{c}\text { Normal } \\
\text { Moderada }\end{array}$ & Media & 0.5 \\
4 & $\begin{array}{l}\text { Exceso de } \\
\text { velocidad } \\
\text { Severidad } \\
\text { Exceso de } \\
\text { velocidad }\end{array}$ & $\begin{array}{c}\text { Bajo } \\
\text { Moderada }\end{array}$ & Alta & 0.7 \\
5 & Bajo & Baja & 1 \\
\hline
\end{tabular}




\subsubsection{Análisis de resultados}

En el COIP se establece que por cada infracción cometida se le deben quitar puntos de la licencia de conducir al conductor. Para validar el modelo de lógica difusa se suman los puntos quitados por cada infracción de tránsito, y se comparan con el promedio de los resultados obtenidos de las 23 señales de tránsito. Se puede observar en la Figura 4.6 que existe una relación, entre los puntos de penalización y la estimación del comportamiento de conducción segura; además, una alta correlación $(r=0.92)$ resultó de las variables antes mencionadas. También se puede observar, que existe una correlación positiva, lo que significa que mientras los valores estimados del comportamiento de conducción (nivel de infracciones de tráfico) aumentan, al conductor se le asigna más puntos de penalización. Cabe señalar que, si bien el reglamento de tránsito del COIP no considera el exceso de velocidad a la hora de emitir una multa (es decir, es lo mismo infringir una señal de pare con un exceso de velocidad de 5 $\mathrm{km} / \mathrm{h} \circ 20 \mathrm{~km} / \mathrm{h}$ ), este modelo lo incluye. Tomando en consideración de que un mayor exceso de velocidad conduce a un accidente de tráfico, se contempló este caso para adaptarlo mejor a la realidad del comportamiento del conductor. Este enfoque ha sido probado en otros países, cuyas leyes permiten considerar el exceso de velocidad para sancionar al conductor (por ejemplo, en España y Australia).

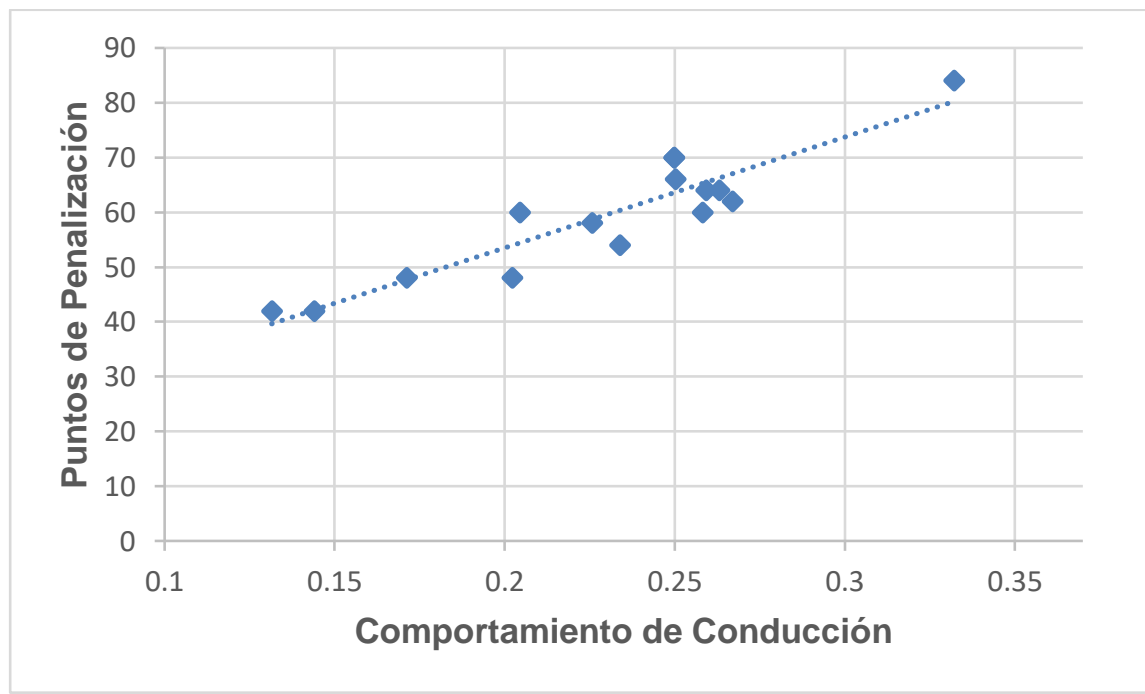

Figura 4.6 Correlación entre los puntos de penalización y la estimación del comportamiento de conducción segura 


\subsubsection{Construcción de modelos computacionales}

\subsubsection{Etiquetado de datos}

El etiquetado de datos implica la evaluación de las observaciones por expertos externos para obtener el "valor de verdad" del conjunto de datos. Este paso es un procedimiento estándar que seguir, cuando el propósito general es imitar las decisiones de los expertos a través de algoritmos o modelos computacionales. Con respecto a los estudios previos descritos en la sección 3.4.3, se observó que el número de clases más utilizada son 2 o 3 categorías. Para este estudio se decidió escoger 3 etiquetas (calmado, normal y agresivo) debido a que un conductor no necesariamente maneja siempre calmado o agresivo en una ruta. Se determino que para etiquetar los datos se lo hará con 15 expertos externos al estudio; y deberán de tener 0.85 o más de acuerdo entre ellos para que la evaluación sea aceptada, caso contrario se deberá realizar otra ronda de evaluación hasta que se llegue a un consentimiento.

Quince expertos del Instituto Universitario de Investigación del Automóvil (INSIA), con una amplia experiencia en investigación de automóviles, evaluaron cada prueba de manejo clasificando a cada conductor como calmado, normal o agresivo para determinar el estilo de conducción. En la Figura 4.7 se puede observar un ejemplo de la evaluación de un experto, en el cual 1 significa calmado, 2 normal y 3 agresivo. Para definir la etiqueta de cada conductor consideramos el valor medio de las 15 evaluaciones. El coeficiente de acuerdo entre evaluadores (ICC) de las 15 evaluaciones tuvo un valor de 0.958. Este coeficiente sugiere una excelente fiabilidad para la evaluación realizada por los expertos [66]. La distribución de las etiquetas terminó con 10 conductores calmados, 22 normales y 18 agresivos. 


\begin{tabular}{|c|c|c|c|c|c|c|}
\hline Conductores & $\begin{array}{c}\text { Aceleración } \\
\text { Promedio } \\
(\mathrm{m} / \mathrm{s} 2)\end{array}$ & $\begin{array}{l}\text { Desaceleración } \\
\text { Promedio }(\mathrm{m} / \mathrm{s} 2)\end{array}$ & $\begin{array}{c}\text { Eventos } \\
\text { Ace }>2 \\
(\mathrm{~m} / \mathrm{s} 2)\end{array}$ & $\begin{array}{c}\text { Eventos } \\
\text { Desa <-2 } \\
(\mathrm{m} / \mathrm{s} 2)\end{array}$ & $\begin{array}{l}\text { Número de } \\
\text { Infracciones }\end{array}$ & $\begin{array}{c}\text { Calificación } \\
\text { Experto }\end{array}$ \\
\hline 1 & 0.6813 & -0.6842 & 5 & 10 & 12 & 2 \\
\hline 2 & 0.5847 & -0.5589 & 4 & 5 & 7 & 2 \\
\hline 3 & 0.6469 & -0.6302 & 13 & 6 & 4 & 3 \\
\hline 4 & 0.5391 & -0.5540 & 9 & 6 & 9 & 2 \\
\hline 5 & 0.7260 & -0.7426 & 10 & 13 & 12 & 3 \\
\hline 6 & 0.6546 & -0.5972 & 18 & 3 & 6 & 3 \\
\hline 7 & 0.6578 & -0.6726 & 18 & 11 & 9 & 3 \\
\hline 8 & 0.6021 & -0.5760 & 8 & 5 & 9 & 2 \\
\hline 9 & 0.5662 & -0.5966 & 10 & 8 & 5 & 3 \\
\hline 10 & 0.6720 & -0.6161 & 10 & 9 & 7 & 3 \\
\hline 11 & 0.6768 & -0.6065 & 12 & 6 & 7 & 3 \\
\hline 12 & 0.5915 & -0.6132 & 10 & 13 & 7 & 3 \\
\hline 13 & 0.7932 & -0.8646 & 13 & 17 & 10 & 3 \\
\hline 14 & 0.5060 & -0.5423 & 0 & 4 & 4 & 1 \\
\hline 15 & 0.6211 & -0.6553 & 8 & 16 & 8 & 3 \\
\hline 16 & 0.4985 & -0.6115 & 1 & 9 & 11 & 2 \\
\hline 17 & 0.5746 & -0.6410 & 8 & 3 & 9 & 3 \\
\hline
\end{tabular}

Figura 4.7 Ejemplo de una evaluación de un experto.

\subsubsection{Generación de datos sintéticos}

La falta de observaciones es un problema de clasificación común en escenarios del mundo real, especialmente en el estilo de conducción, lo que obliga a los investigadores a trabajar con pequeños conjuntos de datos. Una forma de abordar este desafío es produciendo datos sintéticos a través de técnicas de aumento de datos. Este proceso se llama generación de datos sintéticos (ver Sección 2.4.1 para más detalles) y toma los datos etiquetados por los expertos para generar nuevos puntos de datos que sean similares a los puntos de datos reales.

La técnica de sobre muestreó de minorías sintéticas (SMOTE) se aplicó al conjunto de datos. Su objetivo es generar datos sintéticos preservando la distribución original. Esto se hace mediante la creación de un nuevo punto de datos que toma en consideración las características de los puntos cercanos [38]. Usando el programa Weka [118], se aplicó el filtro SMOTE al conjunto de datos.

El nuevo conjunto de datos se generó utilizando las 50 instancias originales, dando como resultado 150 nuevas instancias (50 por categoría). Aunque lo recomendable es dividir el conjunto de datos en entrenamiento, prueba y validación; este trabajo tiene la limitante del tamaño del conjunto datos $(n=50)$. Por este motivo, se decidió dividir el conjunto de datos en un subconjunto para entrenamiento y validación, lo cual es un enfoque válido y utilizado en este tipo de problemas. El conjunto de datos sintéticos $(n=150)$ se utilizará como el 
conjunto de entrenamiento, mientras que el conjunto de datos original $(n=50)$ se utilizará como conjunto de validación.

\subsubsection{Configuración de los modelos}

Esta sección explica el diseño e implementación de cinco modelos, con respecto a la configuración de hiperparámetros y otras características inherentes para cada modelo. Cinco tipos de modelos fueron seleccionados considerando la literatura previa y trabajos relevantes (sección 3.4.4), que indican que la lógica difusa, ANN, SVM, kNN y RF son los modelos más comunes que se utilizan para la clasificación del estilo de conducción. Cada uno de estos tipos de modelo fue entrenado y probado usando diferentes combinaciones de hiperparámetros, creando de esta manera un subconjunto de modelos. De este subconjunto, se seleccionó el modelo con el mejor rendimiento (es decir, el que tenía mejor exactitud). Vale la pena señalar que el diseño y ajuste de hiperparámetros de los modelos se realizó utilizando los datos sintéticos generados en el paso anterior. A continuación, se detallan las configuraciones respectivas para cada modelo.

\section{a. Lógica difusa}

El diseño del modelo de lógica difusa siguió un enfoque basado en datos, motivado por trabajos previos (sección 3.4.4.1) y el asesoramiento de expertos. El modelo difuso se diseñó con un sistema de inferencia difusa Sugeno y se implementó con la herramienta MATLAB. Decidimos usar Sugeno porque da resultados más rápidos que otros sistema de inferencia difusa [45, 46]. Los modelos de lógica difusa funcionan con etiquetas lingüísticas para representar los valores difusos del dominio del problema. En este caso, las etiquetas lingüísticas correspondían a bajo, normal y alto para las cinco variables de entrada. Además de esto, se definieron: 1) las funciones de membresía de las cinco entradas; 2) las etiquetas lingüísticas y 3) un conjunto de reglas para representar el modelo difuso basado en las variables de entradas.

Funciones de pertenencia: se aplicaron funciones de membresía triangular y trapezoidal debido a la facilidad de asignar valores de intervalo a las etiquetas lingüísticas. La Figura 4.8 muestra las funciones de membresía para cada una de las cinco variables de entrada. Como se puede observar en la Figura 4.8, la variable de entrada AccPro se compone de tres funciones de pertenencia, cada una correspondiente a una etiqueta lingüística: una función trapezoidal para 
bajo con un rango de valores de [ 000.450 .65$]$ una función triangular para normal con un rango de valores de [0.4 0.650 .9$]$ y una función trapezoidal para alto con un rango de valores de [0.65 0.91 .21 .2$]$.

La variable DecPro se compone de tres funciones de pertenencia, cada una correspondiente a una etiqueta lingüística: una función trapezoidal para bajo con un rango de valores de $[-0,65-0.40$ 0] una función triangular para normal con un rango de valores de [-0.9 -0.65 -0.4] y una función trapezoidal para alto con un rango de valores de [-1.2 -1.2 -0.9 -0.65].

La variable EveAcc se compone de tres funciones de pertenencia, cada una correspondiente a una etiqueta lingüística: una función trapezoidal para bajo con un rango de valores de [ $\begin{array}{lll}0 & 0 & 4\end{array}$ 8] una función triangular para normal con un rango de valores de [4 8 12] y una función trapezoidal para alto con un rango de valores de [8 1224 24].

La variable EveFre se compone de tres funciones de pertenencia, cada una correspondiente a una etiqueta lingüística: una función trapezoidal para bajo con un rango de valores de [l $\left.\begin{array}{lll}0 & 4 & 4\end{array}\right]$ una función triangular para normal con un rango de valores de [4 8 12] y una función trapezoidal para alto con un rango de valores de [8 122424$]$.

La variable InfrecTra se compone de tres funciones de pertenencia, cada una correspondiente a una etiqueta lingüística: una función trapezoidal para bajo con un rango de valores de [ $\left[\begin{array}{llll}0 & 0 & 0.15 & 0.3\end{array}\right]$ una función triangular para normal con un rango de valores de [0.15 0.30 .45$]$ y una función trapezoidal para alto con un rango de valores de [0.3 0.4511 . 


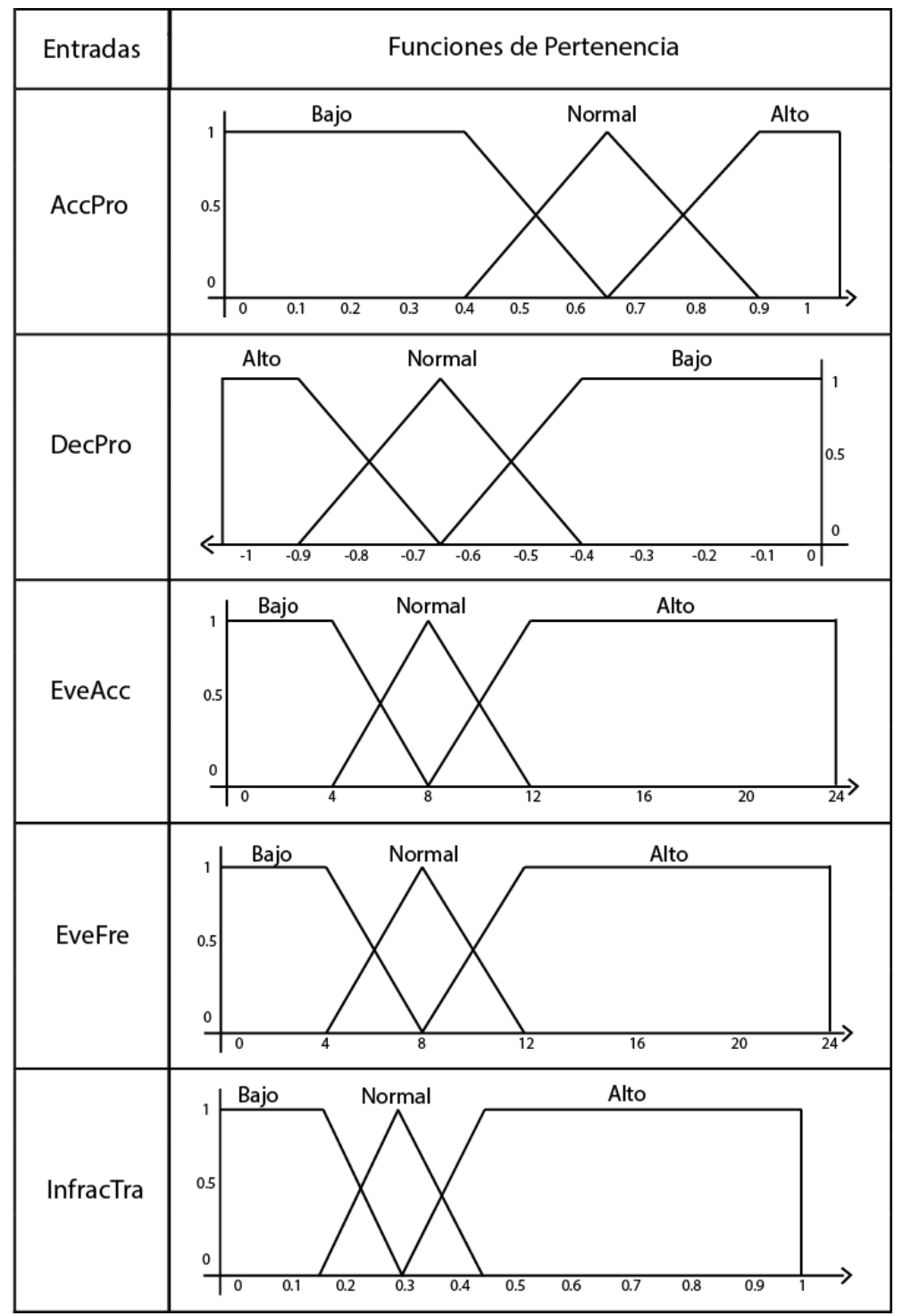

Figura 4.8: Variables de entrada con sus respectivas funciones de pertenencia.

Salida: Para la salida del estilo de conducción, se determinaron tres etiquetas lingüísticas con su función de membresía constante. La etiqueta lingüística calmado tiene un valor de 0 para la función de pertenencia constante, la etiqueta lingüística normal tiene un valor de 0.5 y la etiqueta lingüística agresivo tiene un valor de 1 . Como el modelo difuso da valores entre 0 y 1 , asignamos rangos para determinar la salida del modelo. Por ejemplo, si el valor estimado estaba entre 0 y 0.33 , el estilo de conducción se clasifico como calmado. El siguiente caso es si el valor estimado estaba entre 0.34 y 0.66 , el estilo de conducción se clasifico como normal. Finalmente, si el valor estimado estaba entre 0.67 y 1 , el estilo de conducción se clasifico como agresivos. 
Reglas: la simplicidad y facilidad de un modelo de lógica difusa viene dada por el número de reglas. Para este problema, se definieron 10 reglas para clasificar el estilo de conducción, como se observa en la Tabla 4.3. Cada regla se compone de un antecedente (la entrada y su etiqueta lingüística asignada), un consecuente (la salida del modelo) y un peso. El peso determina la relevancia de la regla, que, en este caso, es crítico para identificar conductores agresivos, debido a que son más propensos a cometer accidentes de tránsito. Por lo tanto, asignamos un peso de 0.8 cuando el consecuente es calmado, un peso de 0.9 cuando el consecuente es normal, y un peso de 1 cuando el consecuente es agresivo.

Tabla 4.3 Reglas definidas a partir de las variables de entrada

\begin{tabular}{|c|c|c|c|c|}
\hline Reglas & \multicolumn{2}{|c|}{ Antecedentes } & Consecuente & Peso \\
\hline 1 & EveAce & $\begin{array}{l}\text { Alto } \\
\text { Alto }\end{array}$ & agresivo & 1 \\
\hline 2 & $\begin{array}{l}\text { EveFre } \\
\text { AvaDec }\end{array}$ & $\begin{array}{l}\text { Alto } \\
\text { Alto }\end{array}$ & agresivo & 1 \\
\hline 3 & AccPro & Bajo & calmado & 0.8 \\
\hline 4 & $\begin{array}{l}\text { EveAce } \\
\text { EveFre }\end{array}$ & $\begin{array}{l}\text { Alto } \\
\text { Alto }\end{array}$ & agresivo & 1 \\
\hline 5 & $\begin{array}{l}\text { AvgDec } \\
\text { EveFre }\end{array}$ & $\begin{array}{l}\text { Bajo } \\
\text { Bajo }\end{array}$ & calmado & 0.8 \\
\hline 6 & $\begin{array}{l}\text { AccPro } \\
\text { AvgDec }\end{array}$ & $\begin{array}{l}\text { Normal } \\
\text { Normal }\end{array}$ & normal & 0.9 \\
\hline 7 & InfracTra & Alto & agresivo & 1 \\
\hline 8 & InfracTra & Bajo & calmado & 0.8 \\
\hline 9 & InfracTra & Normal & normal & 0.9 \\
\hline 10 & $\begin{array}{l}\text { EveAce } \\
\text { EveFre }\end{array}$ & $\begin{array}{l}\text { Normal } \\
\text { Normal }\end{array}$ & normal & 0.9 \\
\hline
\end{tabular}

\section{b. Redes Neuronales Artificiales}

Cuando se diseña un modelo ANN se deben considerar dos factores: 1) la selección del algoritmo de entrenamiento; y 2) la arquitectura de red [119]. En este trabajo, seleccionamos el algoritmo de retropropagación como el algoritmo de entrenamiento, porque ha demostrado dar buenos resultados de clasificación [13, 92]. Para la arquitectura de red, se deben ajustar los siguientes hiperparámetros: i) el número de capas ocultas; ii) la función de activación; iii) el número de iteraciones máximas; iv) la tasa de aprendizaje (Ir); y v) el número de neuronas $(\mathrm{N})$ para la capa oculta. La implementación del modelo ANN se la realizó en la herramienta de RStudio y la librería RSNNS [120]. Se siguió un diseño similar, según lo propuesto por Hornik et al. [121], que implementó un modelo ANN con una capa oculta y la función de activación sigmoidea. Para 
encontrar el modelo con el mejor rendimiento, se entrenaron y probaron varios modelos ANN con diferentes valores de hiperparámetros, realizando una búsqueda cuadrícula para ajustar el número de iteraciones máximas, la tasa de aprendizaje y el número de neuronas. Se estableció las iteraciones máximas en 400, ya que mediante observación se determinó que el error convergía en ese valor. Se seleccionó una tasa de aprendizaje de un rango entre 0.1 y 1 , y el número de neuronas entre una lista de valores $(3,4,10$ y 12) como se sugiere en varios estudios [122-125] (ver Tabla 4.4). Luego de evaluar los diferentes modelos de ANN, con las diferentes combinaciones de hiperparámetros, se encontró dos modelos con la misma exactitud alta (0.86) en comparación con los otros. Por simplicidad, principio de Navaja de Occam [126], se eligió el modelo de ANN que fue entrenado con el valor de tasa de aprendizaje de 0.4 y 4 neuronas ocultas.

Tabla 4.4 Valores de búsqueda cuadricula para redes neuronales artificiales (ANN).

\begin{tabular}{cccc}
\hline Ecuaciones & $\begin{array}{c}\text { Numero de } \\
\text { neuronas }\end{array}$ & Exactitud & Referencia \\
\hline $2 \times N_{i}+1$ & 3 & 0.84 & {$[122]$} \\
$\left(N_{i}+N_{0}\right) / 2$ & 4 & $\mathbf{0 . 8 6}$ & {$[123]$} \\
$\sqrt{N_{i} \times N_{0}}$ & 10 & $\mathbf{0 . 8 6}$ & {$[124]$} \\
$2 N_{i}$ & 12 & 0.84 & {$[125]$} \\
\hline
\end{tabular}

\section{Máquinas de soporte vectorial}

Siguiendo las pautas proporcionadas en guías de implementación de trabajos previos [127, 128], se realizó una búsqueda cuadrícula para ajustar los hiperparámetros del modelo, combinando: 1) la función kernel y 2) los hiperparámetros de la función de costo (C) y gamma (Y). Por lo tanto, se entrenaron varios modelos SVM con todas las combinaciones posibles de valores de hiperparámetros y funciones de núcleo. Los modelos SVM se implementaron utilizando la herramienta RStudio y la biblioteca LIBSVM [129]. La Tabla 4.5 enumera las cuatro funciones de núcleo y el rango de valores para $C$ y y que se usaron para ajustar los hiperparámetros. Se consideró la exactitud para evaluar el rendimiento del modelo. Un modelo SVM entrenado con un núcleo RBF obtuvo la mayor exactitud (0.96). Como varias combinaciones de valores de $\mathrm{C}$ y $\mathrm{y}$ obtuvieron la misma exactitud decidimos elegir valores intermedios para $C\left(2^{5}\right)$ y y $\left(2^{2}\right)$ para evitar un sobreajuste [130]. 
Tabla 4.5 Valores de búsqueda cuadricula para la máquina de soporte vectorial (SVM).

\begin{tabular}{cc} 
Parámetros & Valores \\
\hline funciones kernel & lineal, polinomial, función de base radial (RBF), sigmoid \\
$C$ & {$\left[2^{-5}, 2^{10}\right]$} \\
$\gamma$ & {$\left[2^{-5}, 2^{10}\right]$} \\
\hline
\end{tabular}

\section{d. Bosques aleatorios}

Los bosques aleatorios (RF) son un método de aprendizaje conjunto en el que se entrenan varios árboles de decisión y la predicción final es la moda o el promedio de los árboles de decisión. Para implementar el modelo se deben ajustar dos hiperparámetros: 1) el número de árboles (n) que crecen en el bosque y 2 ) el número de capas $(\mathrm{m})$ para dividir los nodos de cada árbol. El modelo RF se implementó utilizando la herramienta RStudio y la biblioteca randomForest [131]. Como sugiere Breiman [132], no se debe seleccionar un valor alto de $n$, debido que podría hacer que el modelo tenga sobreajuste. Además, elegir un valor mayor de $\mathrm{m}$ aumenta la correlación entre los árboles produciendo resultados similares en la votación. Se realizó una búsqueda en cuadrícula para seleccionar los mejores valores para $n$ y $m$, utilizando valores entre 1 y 500 , y entre 1 y 10, respectivamente. El modelo RF entrenado con $n=100$ y $m=4$ arrojó la mayor precisión (0.92).

\section{e. k-vecinos más cercanos}

El k-vecinos más cercanos es un método no paramétrico utilizado para la clasificación y la regresión, que se considerado el más simple de todos los algoritmos de métodos supervisados. Para implementar el modelo kNN, se necesita configurar 1) el número de vecinos (k) y 2) la función de distancia, que comúnmente se utiliza la función euclidiana [24]. Elegir el k no es una tarea fácil, ya que puede afectar la exactitud de los resultados de clasificación. Por lo tanto, se realizó una búsqueda en cuadrícula para encontrar el mejor valor de k para este conjunto de datos utilizando valores entre 1 y 10 . El valor de $\mathrm{k}=3$ produjo el mejor rendimiento, con una exactitud de 0.92 .

\subsubsection{Evaluación de modelos}

Una vez que los modelos ya están entrenados se debe evaluar el rendimiento en términos de a) métricas de la matriz de confusión (por ejemplo, 
exactitud, puntaje F1, Kappa); y b) prueba estadística (por ejemplo, prueba de Wilcoxon), que se resume en la siguiente sección.

\subsubsection{Métricas de clasificación}

De acuerdo con pautas definidas en trabajos previos [133, 134], la selección de las métricas para evaluar el rendimiento de los modelos depende del conjunto de datos y sus características (por ejemplo, datos balanceados vs no balanceados, tipo de datos), tal como ha sido explicado en la Sección 2.4.1.. Como se está tratando con un conjunto de datos no balanceados, la exactitud no es suficiente para evaluar el rendimiento de los cinco modelos. Por lo tanto, se seleccionaron las métricas de exactitud, puntaje F1, AUC y Kappa para comparar y determinar el mejor modelo. Para obtener las métricas de rendimiento, primero se calcula la matriz de confusión para cada modelo. Luego, se aplicaron las ecuaciones de la sección 2.5.1 y se obtuvo cada una de las métricas mencionadas. La Tabla 4.6 presenta los resultados de exactitud, puntaje F1, AUC y Kappa para cada modelo. Los valores más altos para cada métrica se han destacado en la Tabla 4.6. Según estos resultados, el modelo SVM con un núcleo $\operatorname{RBF}\left(C=2^{5} ; y=2^{2}\right)$ superó a los demás modelos en relación con todas las métricas.

Tabla 4.6 Métricas de evaluación (exactitud, puntuación F1, Área bajo la curva (AUC), Kappa) para cada modelo. $\mathrm{ANN}=$ redes neuronales artificiales; $\mathrm{SVM}=$ Máquinas de soporte vectorial; $\mathrm{RF}=$ Bosques aleatorios; $\mathrm{kNN}=\mathrm{k}$-vecino más cercano.

\begin{tabular}{lcccc}
\hline \multicolumn{1}{c}{ Modelos } & Exactitud & Puntaje F1 & AUC & Kappa \\
\hline Lógica difusa & 0.8800 & 0.8840 & 0.9072 & 0.8106 \\
ANN (sigmoid; $\mathrm{Ir}=0.4 ; \mathrm{N}=4)$ & 0.8600 & 0.8663 & 0.9030 & 0.7807 \\
SVM (RBF, $\left.\mathrm{C}=2^{5} ; \gamma=2^{-2}\right)$ & $\mathbf{0 . 9 6 0 0}$ & $\mathbf{0 . 9 5 9 5}$ & $\mathbf{0 . 9 7 3 0}$ & $\mathbf{0 . 9 3 7 5}$ \\
RF $(\mathrm{n}=100 ; \mathrm{m}=4)$ & 0.9200 & 0.9253 & 0.9451 & 0.8750 \\
kNN $(\mathrm{k}=3)$ & 0.9200 & 0.9253 & 0.9451 & 0.8750 \\
\hline
\end{tabular}

De cada uno de los diferentes tipos de modelo se tomó en consideración el modelo con mejores resultados. La Tabla 4.7 muestra un resumen de estos modelos y sus métricas respectivas. Aunque estos resultados son prometedores con respecto al rendimiento general de los cinco modelos, en este trabajo, la agresividad tiene un mayor impacto en un entorno real, ya que un conductor agresivo es propenso a causar más infracciones de tráfico y accidentes que un conductor normal o calmado. Por lo tanto, se exploró el rendimiento de clasificación para cada clase, con un énfasis particular en la clase agresiva. La 
Tabla 4.7 compara el puntaje F1 y el AUC por categoría. Como se puede observar en esta tabla, los modelos SVM, RF y kNN tienen el mismo rendimiento para estilos de conducción tranquilos $(F 1=0.9524 ; A U C=0.9875)$. Sin embargo, SVM superó a los otros modelos con respecto a los estilos de conducción normal $(\mathrm{F} 1=0.9545 ; \mathrm{AUC}=0.9594)$ y agresivo $(\mathrm{F} 1=0.9714 ; \mathrm{AUC}=0.9722)$.

Tabla 4.7 Puntaje F1 y AUC por cada estilo de conducción

\begin{tabular}{lcccccccccc}
\hline & \multicolumn{2}{c}{ Lógica Difusa } & \multicolumn{2}{c}{ ANN } & \multicolumn{2}{c}{ SVM } & \multicolumn{2}{c}{ KNN } & \multicolumn{2}{c}{ RF } \\
\hline \multicolumn{1}{c}{ Etiqueta } & F1 & AUC & F1 & AUC & F1 & AUC & F1 & AUC & F1 & AUC \\
\hline Calmado & 0.9 & 0.9375 & 0.9091 & 0.9167 & $\mathbf{0 . 9 5 2 4}$ & $\mathbf{0 . 9 8 7 5}$ & $\mathbf{0 . 9 5 2 4}$ & $\mathbf{0 . 9 8 7 5}$ & $\mathbf{0 . 9 5 2 4}$ & $\mathbf{0 . 9 8 7 5}$ \\
Normal & 0.8696 & 0.8831 & 0.8511 & 0.8600 & $\mathbf{0 . 9 5 4 5}$ & $\mathbf{0 . 9 5 9 4}$ & 0.9091 & 0.9189 & 0.9091 & 0.9189 \\
Agresivo & 0.8823 & 0.9011 & 0.8387 & 0.8387 & $\mathbf{0 . 9 7 1 4}$ & $\mathbf{0 . 9 7 2 2}$ & 0.9143 & 0.9289 & 0.9143 & 0.9289 \\
\hline
\end{tabular}

\subsubsection{Prueba de significancia estadística}

Esta investigación utilizó la prueba de significancia estadística Wilcoxon (sign-ranked), puesto que considera una distribución no normal de los datos. Usualmente se utiliza para comparar las diferencias entre dos muestras que están emparejadas o relacionadas [69]. La prueba de Wilcoxon se aplicó con todas las combinaciones posibles entre los cinco modelos considerados en este trabajo. Para esta prueba, la hipótesis nula $\left(\mathrm{H}_{\circ}\right)$ establece que los modelos predicen igual. Para rechazar $\mathrm{H}_{\circ}$ se considera un $\mathrm{p}$-valor $\leq 0.05$, el cual nos indica si existe una diferencia significativa en cuanto a la forma en que los modelos predicen. Los valores $\mathrm{p}$ resultantes de la prueba de significancia, como se muestra en la Tabla 4.8, indican que la manera en que predice el modelo ANN es diferente con respecto a los otros cuatro modelos ( $p$-valor $=0.025$ para todas las pruebas emparejadas ANN).

Tabla 4.8 Valores de p correspondientes a la prueba de significancia de Wilcoxon

\begin{tabular}{lcc} 
Modelo 1 & Modelo 2 & p-valor \\
\hline SVM & ANN & $\mathbf{0 . 0 2 5}$ \\
SVM & Lógica Difusa & 1 \\
SVM & kNN & 1 \\
SVM & RF & 1 \\
ANN & Lógica Difusa & $\mathbf{0 . 0 2 5}$ \\
ANN & kNN & $\mathbf{0 . 0 2 5}$ \\
ANN & RF & $\mathbf{0 . 0 2 5}$ \\
Lógica Difusa & kNN & 1 \\
Lógica Difusa & RF & 1 \\
kNN & RF & 1 \\
\hline
\end{tabular}




\subsection{Resumen}

Este capítulo describió una metodología propuesta para la correcta implementación y evaluación de modelos computacionales que clasifican el estilo de conducción, ya sea en calmado, normal y agresivo. La metodología comprende cuatro etapas: 1) recolección de datos, 2) generación de variables de entrada, 3) construcción del modelo, 4) evaluación. Cabe recalcar que cada una de estas etapas responde a las limitaciones encontradas en trabajos previos, como se indicó en la sección 3.5.

Adicionalmente, este capítulo presentó el diseño e implementación de un modelo correspondiente a detección automática de infracciones de tránsito. Si bien es cierto, algunos trabajos previos han abordado este tema considerando otras variables (por ejemplo, cuando un conductor cambia de carril en lugares no permitidos), este modelo consideró las infracciones de velocidad y aprovechó la facilidad de captura de datos utilizando dos dispositivos como lo son el ODB2 y un teléfono inteligente que cuente con GPS. La estimación de infracciones de tránsito fue utilizada como variable de entrada de los modelos computacionales que predicen el estilo de conducción.

En el siguiente capítulo se discuten los resultados de la metodología, en especial los resultados de clasificación y evaluación de los diferentes modelos computacionales. 



\section{Capítulo 5: Discusión}

Analizar los estilos de conducción a través de autoinformes puede ser inexacto, ya que solo se considera la perspectiva del conductor para determinar la agresividad del conductor [135]. Los investigadores en esta área han propuesto enfoques computacionales para detectar automáticamente los estilos de conducción a partir de los datos del vehículo. Este trabajo presenta un enfoque basado en datos para clasificar los estilos de conducción como tranquilos, normales o agresivos por entrenamiento y probar cinco modelos, siguiendo una metodología sistemática, y encontrar el mejor modelo utilizando métricas de rendimiento de aprendizaje automático y pruebas estadísticas. En las secciones siguientes se discutirá 1) los resultados obtenidos de la evaluación; y 2) la metodología propuesta para la clasificación de estilos de conducción.

\subsection{Evaluación de desempeño y la prueba estadística}

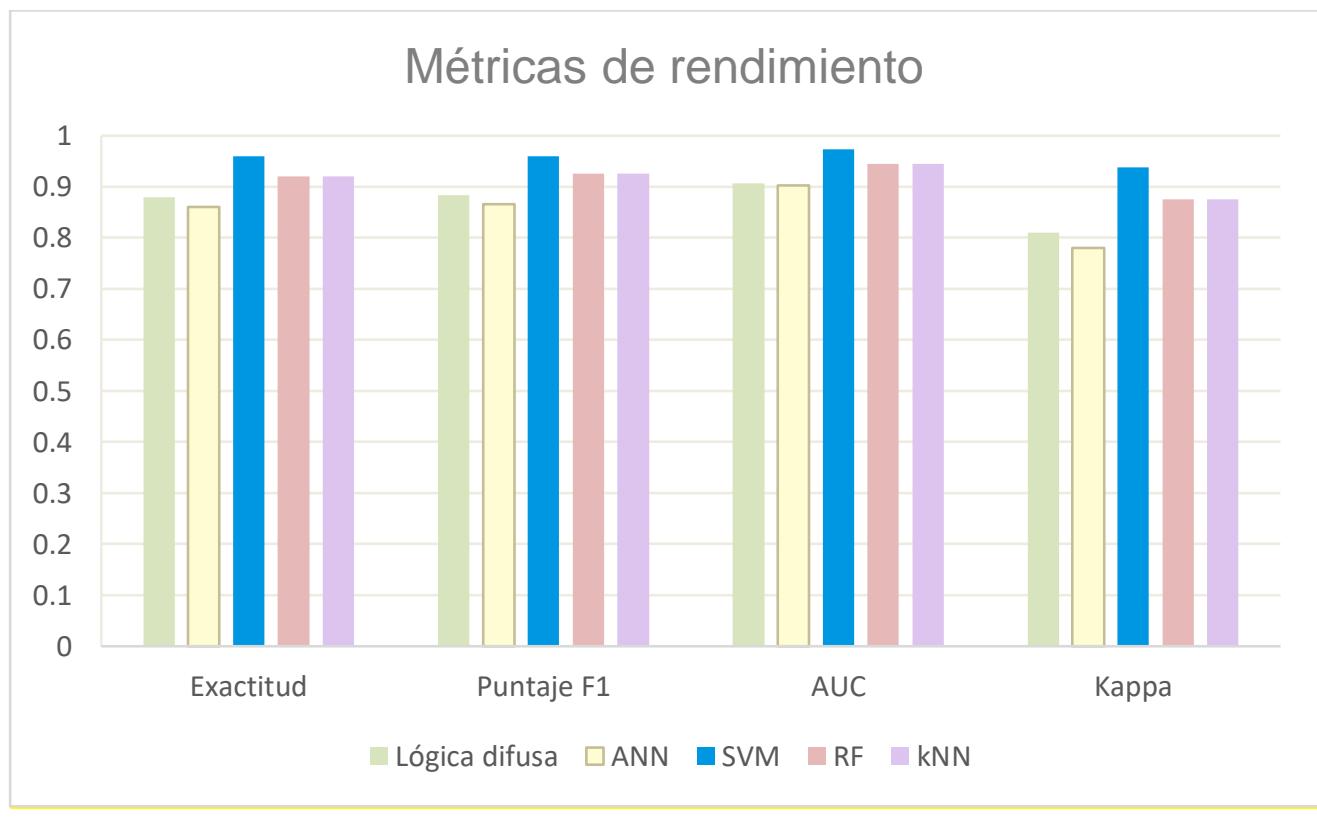

Figura 5.1 Resultados de los modelos computaciones.

Los resultados experimentales (ver Figura 5.1) revelaron que el modelo SVM con núcleo RBF $\left(C=2^{5} ; \gamma=2^{-2}\right)$ tuvo un mejor rendimiento (exactitud de 0.96) que los otros cuatro modelos. Este resultado es consistente con estudios previos reportados por Xue et al. [18] y Wang et al. [17]. En la referencia [18], los 
autores indicaron que el modelo SVM (exactitud $=0.917$ ) superó a los modelos RF, kNN y ANN utilizando aceleración, distancia relativa y características de velocidad relativa para clasificar el estilo de conducción como normales y agresivos de 320 pruebas de conducción en un entorno realista. Del mismo modo, Wang et al. [17] demostró que el modelo SVM con kernel lineal (exactitud $=0.86$ ) tiene mejor rendimiento que un RBF (exactitud $=0.77$ ) que usaba características de velocidad y apertura del acelerador para clasificar los estilos de conducción normales y agresivos. Vale la pena señalar que no podemos generalizar la comparación de estos modelos, porque cada modelo se construyó utilizando diferentes características, resultados y condiciones. Sin embargo, podemos observar que nuestro modelo tiene una mayor exactitud que los modelos reportados en estudios anteriores [17, 18]. Por lo tanto, recomendamos que los futuros investigadores que primero exploren los modelos SVM para clasificar el estilo de conducción.

Los resultados de este estudio indicaron que el modelo $\mathrm{kNN}(\mathrm{k}=3)$ clasificó correctamente el $92 \%$ de las instancias, siendo este resultado más bajo que el de un modelo similar previamente reportado. Por ejemplo, el modelo kNN construido por Vaitkus et al. [110], clasificó correctamente el 98\% de las 110 muestras de manejo como normales o agresivas usando la aceleración longitudinal, la aceleración lateral y la covarianza entre la aceleración longitudinal y lateral como características. Nuevamente, como se mencionó anteriormente, no podemos comparar ambos resultados de exactitud porque cada modelo usa un conjunto de datos diferente. Sin embargo, una posible explicación del bajo rendimiento entre nuestro modelo y el modelo presentado en la referencia [110], puede ser las diferencias en relación con las condiciones experimentales. Los autores mencionaron que el conjunto de datos utilizado para el estudio se recopiló en un entorno controlado (el mismo clima, condiciones de tráfico y ruta) y declararon que esto podría simplificar la tarea de clasificación al distinguir los estilos de conducción normales de agresivos. Por el contrario, nuestro estudio utilizó datos recopilados de dos ciudades en un entorno naturalista, con condiciones de flujo de tráfico diferentes, lo que hace que la tarea de clasificación sea más desafiante. 
El modelo de RF ( $n=100 ; m=4)$ obtuvo una exactitud de 0.92 . Este resultado, sin embargo, no es similar con investigaciones previas. Un trabajo anterior ha informado un rendimiento más alto de un modelo similar, para la tarea de clasificación. Por ejemplo, el modelo $R F(n=200, m=10)$ presentado por Júnior et al. [10] informaron una mayor exactitud (0.99) para clasificar 69 eventos agresivos y no agresivos utilizando la aceleración lateral y longitudinal en un experimento del mundo real. Se deben realizar más estudios para investigar si agregar la aceleración lateral y longitudinal a nuestro espacio de características mejora el rendimiento de clasificación.

En términos del rendimiento del modelo de lógica difusa, los resultados indicaron que este modelo tiene una exactitud de 0.88 . Este resultado difiere de trabajos anteriores. Por ejemplo, el modelo presentado por Castignani [14] alcanzó una exactitud de 0.90 para clasificar estilos de conducción tranquilos y agresivos, mientras que el modelo presentado por Dörr et al. [15] dio un rendimiento de 0.68 para clasificar estilos de conducción como cómodos, normales y deportivos. Debido a la falta de información sobre cómo se construyeron los modelos de estudios anteriores, no podemos proporcionar una explicación de este resultado. Sin embargo, somos conscientes de que el rendimiento del modelo de lógica difusa depende de la experiencia del dominio de los humanos para diseñar reglas difusas y funciones de membresía, así como para interpretar correctamente los resultados difusos para la tarea de clasificación [136].

Finalmente, el modelo ANN (sigmoide; Ir = 0.4; $\mathrm{N}=4$ ) dio una exactitud de 0.86 . Contrastando este resultado con un modelo ANN previo (tanh, $N=2$ ) presentado por Cheng et al. [16], nuestro modelo tiene una exactitud ligeramente inferior (0.892). Los autores utilizaron las características de aceleración, ángulo del pedal y velocidad de los datos recopilados mediante un simulador para clasificar estilos de conducción tranquilos, normales y agresivos en diferentes tipos de carretera (por ejemplo, llano, pendiente).

Además del rendimiento general de los modelos, también estábamos interesados en analizar los resultados de la clasificación entre categorías, con énfasis en la agresividad de los conductores, ya que esto puede ser extremadamente crítico en escenarios realistas. Los resultados de las métricas 
por categoría, tal como se observa en la Figura 5.2, indicaron que SVM también superó a las métricas de rendimiento en comparación con los otros cuatro modelos $(\mathrm{F} 1$-score calmado $=0.9524 ;$ normal $=0.9545$; agresivo $=0.9714$ ) Aunque el conjunto de datos de validación no estaba equilibrado, SVM pudo predecir el $97 \%$ de los conductores agresivos. Las implicaciones de este resultado pueden proporcionar a los investigadores un modelo que se puede aplicar a vehículos inteligentes para la detección temprana de estilos de conducción inseguros.

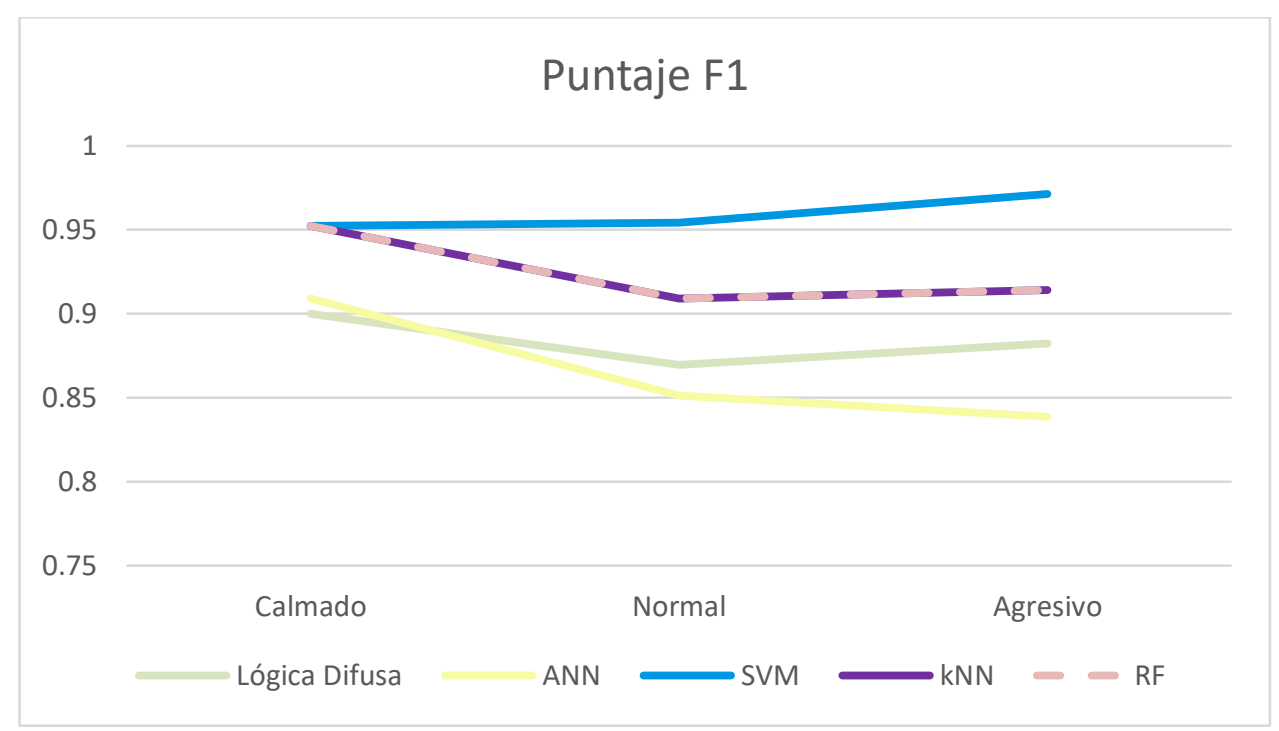

Figura 5.2 Resultado por categoría por modelo computacional

Otro punto por destacar es la prueba de significancia estadística para determinar si hubo una diferencia considerable en la manera de predicción de estos modelos. Los resultados indicaron que las predicciones del modelo ANN (exactitud $=0.86$ ) difiere del resultado de los otros cuatro modelos. Curiosamente, este modelo tuvo el peor rendimiento de todos los modelos. Además, los resultados de la prueba estadística también sugirieron que tenemos la libertad de elegir cualquiera de los otros cuatro modelos para el propósito de clasificación. Sin embargo, deberíamos considerar soluciones más simplistas, como lo señala el principio de Navaja de Occam [126]. En resumen, este principio establece que la solución más simple debe considerarse a partir de un conjunto de soluciones, ya que esto desvanece las suposiciones innecesarias. Por lo tanto, corresponde al investigador definir las características de un modelo simple pero robusto. Por ejemplo, en este trabajo, podríamos argumentar que el número de hiperparámetros a ajustar o su capacidad de interpretación caracteriza la 
simplicidad de un modelo. Si el número de hiperparámetros define el modelo más simple, el kNN debe seleccionarse entre los cuatro modelos. Por otro lado, si la interpretabilidad del modelo se usa para definir la simplicidad del modelo, el modelo de lógica difusa podría elegirse la mejor solución, ya que está construido a partir de un conjunto de reglas simples. Los modelos SVM, kNN y RF pueden considerarse más como una caja negra debido a la dificultad de comprender cómo funciona la lógica interna, principalmente cuando se trabaja con espacios de dimensiones superiores. Debido a que el modelo de SVM fue el mejor clasificando a los conductores agresivo, lo cual en el estilo de conducción es una tarea crítica, se recomienda que este modelo sea utilizando cuando el contexto sea la seguridad vial.

\subsection{Una metodología para la clasificación del estilo de conducción}

La metodología propuesta en este trabajo fue motivada por guías bien establecidas para la implementación de modelos computacionales. Para obtener un buen modelo de clasificación del estilo de conducción, se necesita una metodología convincente para validar los resultados experimentales. De trabajos previos discutidos en la sección 3.4.4, notamos que la mayoría de los modelos de clasificación de estilos de conducción carecían de una validación completa. Por lo tanto, esperamos que esta metodología contribuya al estado del arte, guiando a los investigadores en la implementación de modelos computacionales de una manera estandarizada de selección y evaluación de estos. Se reconocieron algunos aspectos relevantes en la metodología propuesta, como se discute a continuación.

En términos de recopilación de datos, la metodología propuesta se probó utilizando datos de 50 conductores que realizaron una prueba de manejo en un entorno realista. Se analizaron los datos del automóvil en dos ciudades diferentes con rutas similares. Estudios anteriores han informado sobre la implementación de modelos de clasificación utilizando datos recopilados de simuladores [15, 97]. Aunque esto podría ser una ventaja para predecir comportamientos en situaciones de manejo peligrosas, es preferible usar datos capturados de escenarios reales. En este trabajo, se demostró que los datos de un entorno realista podrían clasificarse correctamente, como estilos de 
conducción calmados, normales o agresivos con una exactitud de 0.96 en el modelo SVM.

En la tarea de etiquetar de datos, estudios similares para la clasificación del estilo de conducción han considerado la evaluación de autoinforme a través de cuestionario. Por ejemplo, en el estudio realizado por Hong et al. presentaron una forma de categorizar a los conductores a través del cuestionario DBQ agregando unas preguntan de agresividad. Los conductores que tenían altos puntajes fueron etiquetados como agresivos y los que tuvieron bajo puntaje como calmados, lo que no queda claro es como decidieron el umbral para categorizar entre agresivos y calmados; y como validaron ese proceso [99]. Además, es posible que este tipo de enfoques no se considere la mejor solución, dado que se basan en datos subjetivos [135]. Por otro lado, estudios anteriores han presentado métodos más directos para obtener el valor de verdad de los datos. Por ejemplo, Jiménez et al. informaron la creación de su propio conjunto de datos pidiendo a los conductores que cambien su estilo de conducción a calmado o agresivo antes de la recopilación de datos [95]. Esta manera de recolectar los datos no se apega a la realidad, debido a que obliga al conductor a manejar en una forma particular, en vez de que el conductor maneje con su propio estilo de conducción. Dörr et al. crearon tres conjuntos de datos simulados para etiquetados como estilos de conducción cómodos, normales o deportivos [15]. Este estudio no describió como escogió los umbrales de las variables de entrada para definir la categoría de cada instancia del conjunto de datos. Además, al ser simulados no se puede asegurar que los datos se parezcan al de un entorno real. Otro conjunto de estudios informó el uso de algoritmos de aprendizaje automático no supervisados, como el K-medias, para encontrar patrones grupales comunes que pueden estar relacionados con los estilos de conducción, como calmado o agresivo [14] y normal o agresivo [17, 18]. La limitante de este tipo de estudios es que no se puede validar el proceso realizado para comprobar que el etiquetado de datos fue correcto.

Por el contrario, este estudio proporciona una práctica común en la investigación de modelos computacionales al etiquetar los datos manualmente con la ayuda de expertos en el área. La ventaja que tiene este enfoque es que se puede validar midiendo el grado de acuerdo entre los expertos. El enfoque de 
etiquetar los datos de manera manual se ha explorado poco para la clasificación de estilos de conducción. Esto puede deberse a la laboriosa tarea de etiquetar los datos caso por caso y los costos asociados con esta tarea. Por ejemplo, solo encontramos un estudio en el que 3 expertos aplicaron esta técnica para detectar estilos de conducción, como deportivo, normal y defensivo. Validaron la evaluación realizada por los 3 expertos utilizando el indicador de Alfa de Cronbach el cual obtuvo un valor de 0.85 . En este estudio, 15 expertos externos etiquetaron el conjunto de datos, asignando una etiqueta (calmado, normal o agresivo) a cada conductor. Como se indicó en la Sección 4.2.3.1, se obtuvo un acuerdo entre evaluadores de 0.95 , lo que hace de este conjunto de datos un recurso válido para futuros modelos de exploración y clasificación.

En la metodología propuesta, se aplicó una técnica para generar datos sintéticos para mejorar el rendimiento de predicción de los modelos. Los desafíos de recopilar datos en el vehículo en entornos realistas han llevado a los investigadores a trabajar con pequeños conjuntos de datos, que generalmente presentan una distribución de clase desbalanceada. Para abordar este problema, las técnicas de aumento de datos han demostrado ser una solución rentable para generar puntos de datos similares. En este estudio, los resultados de clasificación utilizando la técnica SMOTE son prometedores. Como se presenta en la Tabla 4.6, las métricas de rendimiento para los cinco modelos indicaron que, utilizando esta técnica, se puede obtener un buen rendimiento, con exactitudes que oscilan entre 0.88 y 0.96 . Hasta donde sabemos, este es el primer trabajo que utiliza esta técnica para la clasificación del estilo de conducción. Esta técnica se ha aplicado y demostrado su eficacia en otras áreas de investigación, como la medicina [137], la clasificación de imágenes [138] y el reconocimiento de voz [139], por nombrar algunos. Somos conscientes de que otras técnicas para generar datos sintéticos también deben considerarse en estudios posteriores.

En relación con el diseño del modelo, la mejor manera de diseñar modelos de predicción es ajustar los hiperparámetros y seleccionar el modelo que produzca el mejor rendimiento. Esta tarea se realizó utilizando la técnica de búsqueda cuadrícula en los hiperparámetros de cada modelo, como se explicó en la sección 2.4.2.2. De esta manera, se está explorando todo el espacio de 
búsqueda (es decir, todos los modelos posibles), entrenando y probando tantos modelos como sea posible. Los estudios anteriores también han utilizado la búsqueda cuadrícula para ajustar los hiperparámetros del modelo, de forma similar a nuestro enfoque. Por ejemplo, Júnior et al. realizó una búsqueda cuadrícula para ajustar los hiperparámetros de los modelos SVM, RF y ANN [10]. En ese estudio, los autores buscaron el mejor modelo SVM ajustando la función del núcleo (lineal, polinomial, RBF, sigmoide), C $\left(2^{-3}, 2^{-1}, 2\right)$ y y $\left(2^{-13}, 2^{-11}, 2^{-9}\right)$ hiperparámetros. Además, buscaron el mejor modelo de RF ajustando el número de árboles (n) de 100 a 200 y el número de capas (m) de 10 a 15. Finalmente, para el modelo ANN, ajustaron el número de capas ocultas de 40, 30, 20 y 10. Los autores informaron que el modelo RF $(n=200, m=15)$ tuvo el mejor rendimiento ( $A \cup C=0.985$ ) entre todos los demás modelos generados mediante la búsqueda cuadrícula. Wang et al. también ajustó los hiperparámetros del modelo SVM a través de la búsqueda cuadrícula [17]. Los valores de C y y variaron de $2^{-5}$ a $2^{10}$ y de $2^{-21}$ a $2^{9}$, respectivamente. Los autores indicaron que el modelo $S V M$ con $C=2^{7}$ y $y=2^{-9}$ tuvo el mejor rendimiento (exactitud de 0.65 ). Como se esperaba, los modelos resultantes de trabajos anteriores difieren de nuestros resultados ( $\mathrm{SVM}$ RBF, $\mathrm{C}=2^{5} ; \mathrm{y}=2^{-2}$ ). Una explicación de esto puede relacionarse con el "teorema de no hay almuerzo gratis" [140], que establece que para comparar varios algoritmos de búsqueda, necesitamos usar la misma información. Sin embargo, este no es el caso con nuestro estudio, ya que los modelos de estudios anteriores se implementaron utilizando diferentes características (aceleración longitudinal y aceleración lateral [10]; aceleración media, frenado y giro [96]).

Con respecto a la evaluación del modelo, vale la pena señalar, como se mencionó en la sección 3.4.4, que la mayoría de los estudios anteriores consideraron solo la exactitud como una métrica de rendimiento. La metodología seguida en este trabajo nos permitió expandir nuestra visión para seleccionar el mejor modelo, primero, eligiendo un modelo basado en varias métricas de rendimiento, y segundo, realizando pruebas estadísticas para determinar el desempeño de estos modelos. Se utilizo las métricas de exactitud, puntaje F1, AUC y Kappa para determinar el modelo con el mejor rendimiento y la prueba de 
significancia estadística de Wilcoxon para determinar si había una diferencia significativa en la forma en que los modelos predijeron. 


\section{Capítulo 6: Conclusiones}

En este trabajo, se propuso una metodología para clasificar el estilo de conducción utilizando modelos computacionales. La metodología propuesta en este trabajo puede verse como una guía para ayudar a los investigadores a tomar decisiones sobre qué modelo es mejor para su problema de clasificación y el más relacionado con la identificación del estilo de conducción. La metodología de cuatro pasos permite a los investigadores lidiar con datos obtenidos de diversos sensores, etiquetar los datos de forma manual a través de expertos y validando dicho proceso con un indicador de acuerdo entre evaluadores, distribución de clases desbalanceada mediante la generación de un conjunto de datos aumentado para fines de entrenamiento de los modelos, clasificación de estilo de conducción a través de diferentes modelos y la evaluación de los clasificadores utilizando métricas de rendimiento (por ejemplo, exactitud, puntaje F1) y análisis de pruebas de significancia estadísticas.

Esta metodología se ilustró clasificando los estilos de conducción a partir de los datos recopilados en dos ciudades diferentes en un entorno realista. Diseñamos e implementamos cinco modelos computacionales (lógica difusa, SVM, ANN, kNN y RF) para determinar cuál tenía el mejor rendimiento para clasificar los estilos de conducción. Por un lado, los resultados experimentales mostraron que SVM resultó en el mejor clasificador para identificar estilos de conducción y, específicamente, conductores agresivos. Por otro lado, los resultados de las pruebas de significación revelaron que había una diferencia significativa en cómo ANN predijo los estilos de conducción. Ambas evaluaciones pueden ayudar a obtener una decisión sobre qué modelo es mejor para esta tarea.

Además, se muestra una solución diferente para identificar las infracciones de tráfico de exceso de velocidad. En este trabajo se planteó un modelo computacional de lógica difusa para identificar la severidad del exceso de velocidad, considerando la velocidad del vehículo y la información de la señal de tráfico como variables de entrada del modelo. Esta forma de identificar las infracciones de tráfico es novedosa, puesto que trabajos anteriores han utilizado 
soluciones más complejas, que, aunque sean precisas, podrían ser más difíciles de implementar en experimentos auténticos. Luego de entrenar el modelo, los resultados del estudio determinaron con una precisión aceptable las infracciones de tráfico, logrando así cumplir con uno de los objetivos del presente trabajo.

\subsection{Limitaciones}

Aunque la metodología propuesta ha demostrado con éxito una evaluación sistemática de modelos de estilos de conducción a partir de datos recopilados en entornos realista, tiene ciertas limitaciones que deben tenerse en cuenta. Si bien los resultados experimentales revelaron que el modelo SVM superó a los otros cuatro modelos, no podemos generalizar estos resultados argumentando que este será el mejor modelo para todos los problemas de clasificación del estilo de conducción. En cambio, el objetivo de la metodología propuesta era guiar a los investigadores a tomar decisiones fundamentadas en la selección y evaluación de su modelo. En trabajos futuros se debería investigar las implicaciones de la metodología propuesta en otros contextos. Otra limitación es el pequeño tamaño del conjunto de datos (50 controladores) y las características de los conductores. El género de los conductores no fue proporcional $(62 \%$ hombres, 38\% mujeres) y los grupos de edad se concentraron en dos grupos (24-34 años: 44\%; 35-54 años: 38\%). Los conductores de entre 18 y 24 años no participaron. Debido a estas limitaciones en la proporción de género y los grupos de edad, no fue posible sacar conclusiones basadas en estas características.

Aunque no se recomienda desarrollar modelos de clasificación utilizando pequeños conjuntos de datos debido a las posibles deficiencias de la generalización del modelo, este trabajo supera esa limitación con la aplicación de una técnica de aumento de datos con fines de entrenamiento ( $n=150)$. Sin embargo, todavía no podemos generalizar nuestros resultados, debido al pequeño número de muestras $(n=50)$ para probar los modelos de estilos de conducción. Finalmente, otra limitación de este trabajo es que no consideramos factores externos que podrían afectar los estilos de manejo, como las condiciones climáticas y la hora del día (día o noche). El trabajo futuro debe explorar la viabilidad de mejorar la clasificación mediante la inclusión de tales variables. 


\subsection{Trabajos futuros}

El trabajo futuro debe evaluar más modelos computacionales, como los modelos ocultos de Markov (HMM), los modelos bayesianos y los modelos de mezcla gaussiana (GMM), que se han implementado en investigaciones relacionadas [99, 141, 142]. Se pueden considerar otras métricas para evaluar modelos de aprendizaje automático, por ejemplo, robustez (si los resultados son estables a lo largo del tiempo), escalabilidad (si el modelo puede manejar volúmenes significativos de datos) o velocidad (tiempo para entrenamiento, prueba y predicción). Por supuesto, la métrica de evaluación elegida depende del objetivo de la investigación. Por ejemplo, si los investigadores están motivados para explorar la entrega de retroalimentación en tiempo real a los conductores, el rendimiento deseable debería discernir la solidez y la velocidad de los modelos. 


\section{Bibliografía}

[1] J. Elander, R. West, and D. French, "Behavioral correlates of individual differences in road-traffic crash risk: An examination of methods and findings," Psychological bulletin, vol. 113, no. 2, pp. 279-279, 1993.

[2] E. Petridou and M. Moustaki, "Human factors in the causation of road traffic crashes," European journal of epidemiology, vol. 16, no. 9, pp. 819826, 2000.

[3] W. H. Organization, Global status report on road safety 2015. World Health Organization, 2015.

[4] L. S. Arnold, "Traffic Safety Culture Index," Washington DC: AAA Foundation for Traffic Safety, 2017.

[5] D. J. Lewis, J. D. Russell, and C. S. Tuttle, "Driver feedback to improve vehicle performance," ed: Google Patents, 2010.

[6] J. Peng, B. Fields, P. Rutkowski, and B. Bowne, "Systems and methods for providing driver feedback using a handheld mobile device," ed: Google Patents, 2011.

[7] C. M. Martinez, M. Heucke, F.-Y. Wang, B. Gao, and D. Cao, "Driving style recognition for intelligent vehicle control and advanced driver assistance: A survey," IEEE Transactions on Intelligent Transportation Systems, vol. 19, no. 3, pp. 666-676, 2017.

[8] G. Castignani, R. Frank, and T. Engel, "An evaluation study of driver profiling fuzzy algorithms using smartphones," in 2013 21st IEEE International Conference on Network Protocols (ICNP), 2013 2013, pp. 16.

[9] A. Doshi and M. M. Trivedi, "Examining the impact of driving style on the predictability and responsiveness of the driver: Real-world and simulator analysis," in 2010 IEEE Intelligent Vehicles Symposium, 2010 2010, pp. 232-237.

[10] J. F. Júnior et al., "Driver behavior profiling: An investigation with different smartphone sensors and machine learning," PLoS one, vol. 12, no. 4, pp. e0174959-e0174959, 2017.

[11] G. Meiring and $H$. Myburgh, "A review of intelligent driving style analysis systems and related artificial intelligence algorithms," Sensors, vol. 15, no. 12, pp. 30653-30682, 2015.

[12] S. Kanarachos, S.-R. G. Christopoulos, and A. Chroneos, "Smartphones as an integrated platform for monitoring driver behaviour: The role of sensor fusion and connectivity," Transportation Research Part C: Emerging Technologies, https://doi.org/10.1016/j.trc.2018.03.023 vol. 95, pp. 867-882, $2018 . \quad$ [Online]. Available: http://www.sciencedirect.com/science/article/pii/S0968090X18303954.

[13] J. E. Meseguer, C. T. Calafate, J. C. Cano, and P. Manzoni, "Drivingstyles: A smartphone application to assess driver behavior," in 2013 IEEE Symposium on Computers and Communications (ISCC), 2013 2013, pp. 000535-000540.

[14] G. Castignani, T. Derrmann, R. Frank, and T. Engel, "Driver behavior profiling using smartphones: A low-cost platform for driver monitoring," 
IEEE Intelligent Transportation Systems Magazine, vol. 7, no. 1, pp. 91102, 2015.

[15] D. Dörr, D. Grabengiesser, and F. Gauterin, "Online driving style recognition using fuzzy logic," in 17th International IEEE Conference on Intelligent Transportation Systems (ITSC), 2014/10 2014, pp. 1021-1026.

[16] Z.-J. Cheng, L.-W. Jeng, and K. Li, "Behavioral Classification of Drivers for Driving Efficiency Related ADAS Using Artificial Neural Network," in 2018 IEEE International Conference on Advanced Manufacturing (ICAM), 2018 2018, pp. 173-176.

[17] W. Wang, J. Xi, A. Chong, and L. Li, "Driving style classification using a semisupervised support vector machine," IEEE Transactions on HumanMachine Systems, vol. 47, no. 5, pp. 650-660, 2017.

[18] Q. Xue, K. Wang, J. J. Lu, and Y. Liu, "Rapid Driving Style Recognition in Car-Following Using Machine Learning and Vehicle Trajectory Data," Journal of Advanced Transportation, vol. 2019, 2019.

[19] M. M. Bejani and M. Ghatee, "A context aware system for driving style evaluation by an ensemble learning on smartphone sensors data," Transportation Research Part C: Emerging Technologies, vol. 89, pp. 303320, 2018.

[20] J. Smith, Machine Learning Systems: Designs that Scale. Manning Publications Co., 2018.

[21] V. Lakshmanan, S. Robinson, and M. Munn, Machine Learning Design Patterns. O'Reilly Media, Inc., 2020.

[22] P. Harrington, Machine learning in action. Manning Publications Co., 2012.

[23] R. Sapsford, Survey research. Sage, 2006.

[24] J. Han, J. Pei, and M. Kamber, Data mining: concepts and techniques. Elsevier, 2011.

[25] D. C. Montgomery, Design and analysis of experiments. John wiley \& sons, 2017.

[26] T. Arampatzis, J. Lygeros, and S. Manesis, "A survey of applications of wireless sensors and wireless sensor networks," in Proceedings of the 2005 IEEE International Symposium on, Mediterrean Conference on Control and Automation Intelligent Control, 2005., 2005: IEEE, pp. 719724.

[27] J. Müller, S. Fàbregues, E. A. Guenther, and M. J. Romano, "Using Sensors in Organizational Research-Clarifying Rationales and Validation Challenges for Mixed Methods," Frontiers in psychology, vol. 10, p. 1188, 2019.

[28] I. Mierswa and K. Morik, "Automatic feature extraction for classifying audio data," Machine learning, vol. 58, no. 2-3, pp. 127-149, 2005.

[29] A. Mueen, S. Baba, and R. Zainuddin, "Multilevel feature extraction and Xray image classification," Journal of Applied Sciences, vol. 7, no. 8, pp. 1224-1229, 2007.

[30] Y. Masoudi-Sobhanzadeh, H. Motieghader, and A. Masoudi-Nejad, "FeatureSelect: a software for feature selection based on machine learning approaches," BMC bioinformatics, vol. 20, no. 1, p. 170, 2019.

[31] A. Jović, K. Brkić, and N. Bogunović, "A review of feature selection methods with applications," in 2015 38th international convention on information and communication technology, electronics and microelectronics (MIPRO), 2015: leee, pp. 1200-1205. 
[32] Y. Roh, G. Heo, and S. E. Whang, "A survey on data collection for machine learning: a big data-ai integration perspective," IEEE Transactions on Knowledge and Data Engineering, 2019.

[33] K. A. Hallgren, "Computing inter-rater reliability for observational data: an overview and tutorial," Tutorials in quantitative methods for psychology, vol. 8, no. 1, p. 23, 2012.

[34] K. O. McGraw and S. P. Wong, "Forming inferences about some intraclass correlation coefficients," Psychological methods, vol. 1, no. 1, p. 30, 1996.

[35] D. A. Van Dyk and X.-L. Meng, "The art of data augmentation," Journal of Computational and Graphical Statistics, vol. 10, no. 1, pp. 1-50, 2001.

[36] S. C. Wong, A. Gatt, V. Stamatescu, and M. D. McDonnell, "Understanding data augmentation for classification: when to warp?," in 2016 international conference on digital image computing: techniques and applications (DICTA), 2016: IEEE, pp. 1-6.

[37] A. Amin et al., "Comparing oversampling techniques to handle the class imbalance problem: A customer churn prediction case study," IEEE Access, vol. 4, pp. 7940-7957, 2016.

[38] N. V. Chawla, K. W. Bowyer, L. O. Hall, and W. P. Kegelmeyer, "SMOTE: synthetic minority over-sampling technique," Journal of artificial intelligence research, vol. 16, pp. 321-357, 2002.

[39] H. He, Y. Bai, E. A. Garcia, and S. Li, "ADASYN: Adaptive synthetic sampling approach for imbalanced learning," in 2008 IEEE international joint conference on neural networks (IEEE world congress on computational intelligence), 2008: IEEE, pp. 1322-1328.

[40] E. Alpaydin, Introduction to machine learning. MIT press, 2020.

[41] A. Kaplan and M. Haenlein, "Siri, Siri, in my hand: Who's the fairest in the land? On the interpretations, illustrations, and implications of artificial intelligence," Business Horizons, vol. 62, no. 1, pp. 15-25, 2019.

[42] I. Contreras and J. Vehi, "Artificial intelligence for diabetes management and decision support: literature review," Journal of medical Internet research, vol. 20, no. 5, p. e10775, 2018.

[43] L. Á. Munárriz, Fundamentos de inteligencia artificial. Editum, 1994.

[44] T. J. Ross, Fuzzy logic with engineering applications. John Wiley \& Sons, 2009.

[45] K. Guney and N. Sarikaya, "Comparison of Mamdani and Sugeno fuzzy inference system models for resonant frequency calculation of rectangular microstrip antennas," Progress In Electromagnetics Research, vol. 12, pp. 81-104, 2009.

[46] J. J. Jassbi, P. J. A. Serra, R. A. Ribeiro, and A. Donati, "A comparison of mandani and sugeno inference systems for a space fault detection application," in Automation Congress, 2006. WAC'06. World, 2006 2006, pp. 1-8.

[47] S. S. Haykin and others, Neural networks and learning machines/Simon Haykin. New York: Prentice Hall, 2009.

[48] A. Krizhevsky, I. Sutskever, and G. E. Hinton, "Imagenet classification with deep convolutional neural networks," in Advances in neural information processing systems, 2012 2012, pp. 1097-1105.

[49] A. Graves, A.-r. Mohamed, and G. Hinton, "Speech recognition with deep recurrent neural networks," in 2013 IEEE international conference on acoustics, speech and signal processing, 2013 2013, pp. 6645-6649. 
[50] J. Khan et al., "Classification and diagnostic prediction of cancers using gene expression profiling and artificial neural networks," Nature medicine, vol. 7, no. 6, pp. 673-673, 2001.

[51] C. C. Aggarwal, Neural networks and deep learning. Springer, 2018.

[52] B. E. Boser, I. M. Guyon, and V. N. Vapnik, "A training algorithm for optimal margin classifiers," in Proceedings of the fifth annual workshop on Computational learning theory, 1992 1992, pp. 144-152.

[53] W. M. Campbell, D. E. Sturim, D. A. Reynolds, and A. Solomonoff, "SVM based speaker verification using a GMM supervector kernel and NAP variability compensation," in 2006 IEEE International Conference on Acoustics Speech and Signal Processing Proceedings, 2006 2006, vol. 1, pp. I-I.

[54] T. Joachims, N. Cristianini, and J. Shawe-Taylor, "Composite kernels for hypertext categorisation," in ICML, 2001 2001, vol. 1, pp. 250-257.

[55] G. Guo, S. Z. Li, and K. Chan, "Face recognition by support vector machines," in Proceedings fourth IEEE international conference on automatic face and gesture recognition (cat. no. PR00580), 2000 2000, pp. 196-201.

[56] I. Steinwart and A. Christmann, Support vector machines. Springer Science \& Business Media, 2008.

[57] W. S. Noble, "What is a support vector machine?," Nature biotechnology, vol. 24, no. 12, pp. 1565-1565, 2006.

[58] L. Breiman, "Random forests," Machine learning, vol. 45, no. 1, pp. 5-32, 2001.

[59] A. Verikas, A. Gelzinis, and M. Bacauskiene, "Mining data with random forests: A survey and results of new tests," Pattern recognition, vol. 44, no. 2, pp. 330-349, 2011.

[60] L. Breiman, "Consistency for a simple model of random forests," 2004.

[61] E. Fix and J. L. Hodges Jr, "Discriminatory analysis-nonparametric discrimination: consistency properties," 1951.

[62] J. Snoek, H. Larochelle, and R. P. Adams, "Practical bayesian optimization of machine learning algorithms," in Advances in neural information processing systems, 2012, pp. 2951-2959.

[63] Y. Gao, Y. Guo, Y. Shi, S. Liao, J. Lian, and D. Shen, "Image-Guided Radiotherapy with Machine Learning," in Machine Learning in Radiation Oncology: Springer, 2015, pp. 157-192.

[64] Y. Yao, L. Rosasco, and A. Caponnetto, "On early stopping in gradient descent learning," Constructive Approximation, vol. 26, no. 2, pp. 289-315, 2007.

[65] J. Cohen, "A coefficient of agreement for nominal scales," Educational and psychological measurement, vol. 20, no. 1, pp. 37-46, 1960.

[66] J. R. Landis and G. G. Koch, "An application of hierarchical kappa-type statistics in the assessment of majority agreement among multiple observers," Biometrics, pp. 363-374, 1977.

[67] D. W. Hosmer Jr, S. Lemeshow, and R. X. Sturdivant, Applied logistic regression. John Wiley \& Sons, 2013.

[68] N. Japkowicz and M. Shah, Evaluating learning algorithms: a classification perspective. Cambridge University Press, 2011.

[69] F. Wilcoxon, "Individual comparisons by ranking methods," Biometrics bulletin, vol. 1, no. 6, pp. 80-83, 1945. 
[70] F. Sagberg, Selpi, G. F. Bianchi Piccinini, and J. Engström, "A review of research on driving styles and road safety," Human factors, vol. 57, no. 7, pp. 1248-1275, 2015.

[71] O. Taubman-Ben-Ari, M. Mikulincer, and O. Gillath, "The multidimensional driving style inventory-scale construct and validation," Accident Analysis \& Prevention, vol. 36, no. 3, pp. 323-332, 2004.

[72] S. A. Useche, B. Cendales, F. Alonso, J. C. Pastor, and L. Montoro, "Validation of the Multidimensional Driving Style Inventory (MDSI) in professional drivers: how does it work in transportation workers?," Transportation research part F: traffic psychology and behaviour, vol. 67, pp. 155-163, 2019.

[73] O. Taubman-Ben-Ari and V. Skvirsky, "The multidimensional driving style inventory a decade later: Review of the literature and re-evaluation of the scale," Accident Analysis \& Prevention, vol. 93, pp. 179-188, 2016.

[74] E. Ericsson, "Variability in urban driving patterns," Transportation Research Part D: Transport and Environment, vol. 5, no. 5, pp. 337-354, 2000.

[75] M. Kilpeläinen and H. Summala, "Effects of weather and weather forecasts on driver behaviour," Transportation research part F: traffic psychology and behaviour, vol. 10, no. 4, pp. 288-299, 2007.

[76] R. Paleti, N. Eluru, and C. R. Bhat, "Examining the influence of aggressive driving behavior on driver injury severity in traffic crashes," Accident Analysis \& Prevention, vol. 42, no. 6, pp. 1839-1854, 2010.

[77] L. Tasca, A review of the literature on aggressive driving research. Ontario Advisory Group on Safe Driving Secretariat, Road User Safety Branch, Ontario Ministry of Transportation Ontario, Canada, 2000.

[78] T. Neuman et al., "A guide for addressing aggressive-driving collisions," ed: Report, 2003.

[79] N. H. T. S. Administration, "Aggressive driving enforcement: Strategies for implementing best practices," DOT HS, vol. 809, p. 031, 2000.

[80] J. A. Michon, "A critical view of driver behavior models: what do we know, what should we do?," in Human behavior and traffic safety: Springer, 1985, pp. 485-524.

[81] J. Reason, A. Manstead, S. Stradling, J. Baxter, and K. Campbell, "Errors and violations on the roads: a real distinction?," Ergonomics, vol. 33, no. 10-11, pp. 1315-1332, 1990.

[82] E. Gulian, G. Matthews, A. I. Glendon, D. Davies, and L. Debney, "Dimensions of driver stress," Ergonomics, vol. 32, no. 6, pp. 585-602, 1989.

[83] J. L. Deffenbacher, E. R. Oetting, and R. S. Lynch, "Development of a driving anger scale," Psychological reports, vol. 74, no. 1, pp. 83-91, 1994.

[84] S. T. Cordazzo, C. T. Scialfa, K. Bubric, and R. J. Ross, "The driver behaviour questionnaire: A north American analysis," Journal of safety research, vol. 50, pp. 99-107, 2014.

[85] J. De Winter and D. Dodou, "The Driver Behaviour Questionnaire as a predictor of accidents: A meta-analysis," Journal of safety research, vol. 41, no. 6, pp. 463-470, 2010.

[86] G. Matthews, L. Dorn, and A. I. Glendon, "Personality correlates of driver stress," Personality and Individual Differences, vol. 12, no. 6, pp. 535-549, 1991. 
[87] S. Westerman and D. Haigney, "Individual differences in driver stress, error and violation," Personality and Individual Differences, vol. 29, no. 5, pp. 981-998, 2000.

[88] Y. Yasak and B. Esiyok, "Anger amongst Turkish drivers: Driving Anger Scale and its adapted, long and short version," Safety Science, vol. 47, no. 1, pp. 138-144, 2009.

[89] A. af Wåhlberg, "Driver Behaviour and Accident Research Methodology: Unresolved Problems," ed: Ashgate, 2009.

[90] L. Evans, Traffic safety. 2004.

[91] R. Chhabra, S. Verma, and C. R. Krishna, "A survey on driver behavior detection techniques for intelligent transportation systems," in 2017 7th International Conference on Cloud Computing, Data Science \& Engineering-Confluence, 2017: IEEE, pp. 36-41.

[92] P. Brombacher, J. Masino, M. Frey, and F. Gauterin, "Driving event detection and driving style classification using artificial neural networks," in 2017 IEEE International Conference on Industrial Technology (ICIT), 2017 2017, pp. 997-1002.

[93] A. Mudgal, S. Hallmark, A. Carriquiry, and K. Gkritza, "Driving behavior at a roundabout: A hierarchical Bayesian regression analysis," Transportation research part D: transport and environment, vol. 26, pp. 2026, 2014.

[94] A. Vilaca, P. Cunha, and A. L. Ferreira, "Systematic literature review on driving behavior," in 2017 IEEE 20th International Conference on Intelligent Transportation Systems (ITSC), 2017 2017, pp. 1-8.

[95] F. Jiménez, J. C. Amarillo, J. E. Naranjo, F. Serradilla, and A. Díaz, "Energy consumption estimation in electric vehicles considering driving style," in 2015 IEEE 18th International Conference on Intelligent Transportation Systems, 2015, pp. 101-106.

[96] M. Van Ly, S. Martin, and M. M. Trivedi, "Driver classification and driving style recognition using inertial sensors," in 2013 IEEE Intelligent Vehicles Symposium (IV), 2013 2013, pp. 1040-1045.

[97] J. O. López, A. C. C. Pinilla, and others, "Driver behavior classification model based on an intelligent driving diagnosis system," in 15th International IEEE Conference on Intelligent Transportation Systems, 2012 2012, pp. 894-899.

[98] T. Osafune, T. Takahashi, N. Kiyama, T. Sobue, H. Yamaguchi, and T. Higashino, "Analysis of accident risks from driving behaviors," International Journal of Intelligent Transportation Systems Research, vol. 15, no. 3, pp. 192-202, 2017.

[99] J.-H. Hong, B. Margines, and A. K. Dey, "A smartphone-based sensing platform to model aggressive driving behaviors," in Proceedings of the 32nd annual ACM conference on Human factors in computing systems, 2014 2014, pp. 4047-4056.

[100] Y. L. Murphey, R. Milton, and L. Kiliaris, "Driver's style classification using jerk analysis," in 2009 IEEE Workshop on Computational Intelligence in Vehicles and Vehicular Systems, 2009 2009, pp. 23-28.

[101] N. Aliane, J. Fernández, S. Bemposta, and M. Mata, "Traffic violation alert and management," in Intelligent Transportation Systems (ITSC), 2011 14th International IEEE 2011 2011, pp. 1716-1720. 
[102] O. Nejati, "Smart recording of traffic violations via M-RFID," in 2011 7th International Conference on Wireless Communications, Networking and Mobile Computing, 2011: IEEE, pp. 1-4.

[103] Z. Cheng, L. Jeng, and K. Li, "Behavioral Classification of Drivers for Driving Efficiency Related ADAS Using Artificial Neural Network," in 2018 IEEE International Conference on Advanced Manufacturing (ICAM), 2018/11 2018, pp. 173-176.

[104] A. Aljaafreh, N. Alshabatat, and M. S. N. Al-Din, "Driving style recognition using fuzzy logic," in 2012 IEEE International Conference on Vehicular Electronics and Safety (ICVES), 2012 2012, pp. 460-463.

[105] J. A. Hartigan and M. A. Wong, "Algorithm AS 136: A k-means clustering algorithm," Journal of the royal statistical society. series $c$ (applied statistics), vol. 28, no. 1, pp. 100-108, 1979.

[106] H. Valizadegan, Q. Nguyen, and M. Hauskrecht, "Learning classification models from multiple experts," Journal of biomedical informatics, vol. 46, no. 6, pp. 1125-1135, 2013.

[107] G. Li, S. E. Li, B. Cheng, and P. Green, "Estimation of driving style in naturalistic highway traffic using maneuver transition probabilities," Transportation Research Part C: Emerging Technologies, vol. 74, pp. 113125, 2017.

[108] C. Zhang, M. Patel, S. Buthpitiya, K. Lyons, B. Harrison, and G. D. Abowd, "Driver classification based on driving behaviors," in Proceedings of the 21st International Conference on Intelligent User Interfaces, 20162016 , pp. 80-84.

[109] N. Karginova, S. Byttner, and M. Svensson, "Data-driven methods for classification of driving styles in buses," 2012.

[110] V. Vaitkus, P. Lengvenis, and G. Žylius, "Driving style classification using long-term accelerometer information," in 2014 19th International Conference on Methods and Models in Automation and Robotics (MMAR), 2014 2014, pp. 641-644.

[111] H. Eren, S. Makinist, E. Akin, and A. Yilmaz, "Estimating driving behavior by a smartphone," in 2012 IEEE Intelligent Vehicles Symposium, 2012 2012, pp. 234-239.

[112] A. Sathyanarayana, P. Boyraz, and J. H. L. Hansen, "Driver behavior analysis and route recognition by hidden Markov models," in 2008 IEEE International Conference on Vehicular Electronics and Safety, 2008 2008, pp. 276-281.

[113] D.-W. Koh and H.-B. Kang, "Smartphone-based modeling and detection of aggressiveness reactions in senior drivers," in 2015 IEEE Intelligent Vehicles Symposium (IV), 2015 2015, pp. 12-17.

[114] T.

Pro. "https://play.google.com/store/apps/details?id=org.prowl.torque\&hl=en U S\&gl=US." (accessed 15/10/2019).

[115] J. Paefgen, F. Kehr, Y. Zhai, and F. Michahelles, "Driving behavior analysis with smartphones: insights from a controlled field study," in Proceedings of the 11th International Conference on mobile and ubiquitous multimedia, 2012 2012, pp. 36-36.

[116] C. C. Robusto, "The cosine-haversine formula," The American Mathematical Monthly, vol. 64, no. 1, pp. 38-40, 1957.

[117] Código Orgánico Integral Penal, 2014. 
[118] M. Hall, E. Frank, G. Holmes, B. Pfahringer, P. Reutemann, and I. H. Witten, "The WEKA data mining software: an update," ACM SIGKDD explorations newsletter, vol. 11, no. 1, pp. 10-18, 2009.

[119] D. R. Hush, "Classification with neural networks: a performance analysis," in Proceedings of the IEEE international conference on systems engineering, 1989 1989, pp. 277-280.

[120] C. N. Bergmeir, J. M. Benítez Sánchez, and others, "Neural networks in R using the Stuttgart neural network simulator: RSNNS," 20122012.

[121] K. Hornik, M. Stinchcombe, and H. White, "Multilayer feedforward networks are universal approximators," Neural networks, vol. 2, no. 5, pp. 359-366, 1989.

[122] C. Wang, "A theory of generalization in learning machines with neural network applications," 1994.

[123] B. D. Ripley, "Statistical aspects of neural networks," Networks and chaos_statistical and probabilistic aspects, vol. 50, pp. 40-123, 1993.

[124] I. Kaastra and M. Boyd, "Designing a neural network for forecasting financial and economic time series," Neurocomputing, vol. 10, no. 3, pp. 215-236, 1996.

[125] R. Hecht-Nielsen, "Kolmogorov's mapping neural network existence theorem," in Proceedings of the IEEE International Conference on Neural Networks III, 1987 1987, pp. 11-13.

[126] A. Blumer, A. Ehrenfeucht, D. Haussler, and M. K. Warmuth, "Occam's razor," Information processing letters, vol. 24, no. 6, pp. 377-380, 1987.

[127] A. Ben-Hur and J. Weston, "A user's guide to support vector machines," in Data mining techniques for the life sciences: Springer, 2010, pp. 223239.

[128] C. J. Burges, "A tutorial on support vector machines for pattern recognition," Data mining and knowledge discovery, vol. 2, no. 2, pp. 121167, 1998.

[129] C.-C. Chang, "'" LIBSVM: a library for support vector machines," ACM Transactions on Intelligent Systems and Technology, 2: 27: 1--27: 27, 2011," http://www. csie. ntu. edu. tw/ cjlin/libsvm, vol. 2, 2011.

[130] Y. Zhou and S. Pei, "An Effective Adaptive Multi-objective Particle Swarm for Multimodal Constrained Function Optimization," JCP, vol. 5, no. 8, pp. 1144-1151, 2010.

[131] A. Liaw, M. Wiener, and others, "Classification and regression by randomForest," $R$ news, vol. 2, no. 3, pp. 18-22, 2002.

[132] L. Breiman, "Bagging predictors," Machine learning, vol. 24, no. 2, pp. 123140, 1996.

[133] M. Sokolova and G. Lapalme, "A systematic analysis of performance measures for classification tasks," Information Processing \& Management, vol. 45, no. 4, pp. 427-437, 2009.

[134] J. Shreve, H. Schneider, and O. Soysal, "A methodology for comparing classification methods through the assessment of model stability and validity in variable selection," Decision Support Systems, vol. 52, no. 1, pp. 247-257, 2011.

[135] A. afWahlberg, Driver behaviour and accident research methodology: unresolved problems. Boca Raton, Florida, USA: CRC Press, 2017. 
[136] S. S. Godil, M. S. Shamim, S. A. Enam, and U. Qidwai, "Fuzzy logic: A "simple" solution for complexities in neurosciences?," Surgical neurology international, vol. 2, 2011.

[137] C. K. Enders, "A primer on the use of modern missing-data methods in psychosomatic medicine research," Psychosomatic medicine, vol. 68, no. 3, pp. 427-436, 2006.

[138] L. Perez and J. Wang, "The effectiveness of data augmentation in image classification using deep learning," arXiv preprint arXiv:1712.04621, 2017.

[139] T. Ko, V. Peddinti, D. Povey, and S. Khudanpur, "Audio augmentation for speech recognition," in Sixteenth Annual Conference of the International Speech Communication Association, 20152015.

[140] Y.-C. Ho and D. L. Pepyne, "Simple explanation of the no-free-lunch theorem and its implications," Journal of optimization theory and applications, vol. 115, no. 3, pp. 549-570, 2002.

[141] D. Mitrovic, "Reliable method for driving events recognition," IEEE transactions on intelligent transportation systems, vol. 6, no. 2, pp. 198205, 2005.

[142] C. Miyajima et al., "Driver modeling based on driving behavior and its evaluation in driver identification," Proceedings of the IEEE, vol. 95, no. 2, pp. 427-437, 2007. 


\section{Apéndice}

Código del modelo borroso en MATLAB

[System]

Name='AgreTVPRUEBA2'

Type='sugeno'

Version $=2.0$

Numlnputs $=5$

NumOutputs $=1$

NumRules $=9$

AndMethod='prod'

OrMethod='probor'

ImpMethod='prod'

AggMethod='sum'

DefuzzMethod='wtaver'

[Input1]

Name='NumEvenAce'

Range $=\left[\begin{array}{ll}0 & 30\end{array}\right]$

NumMFs $=3$

MF1='Low':'trapmf',[[0 004 8]

MF2='Normal':'trimf',[[4 8 12]

MF3='High':'trapmf',[8 1230 30]

[Input2]

Name='Acceleration'

Range $=\left[\begin{array}{ll}0 & 1\end{array}\right]$

NumMFs=3

MF1='Low':'trapmf',[0 00.4 0.65]

MF2='Normal':'trimf',[0.4 0.65 0.9]

MF3='High':'trapmf',[0.65 0.911 1.2]

[Input3] 


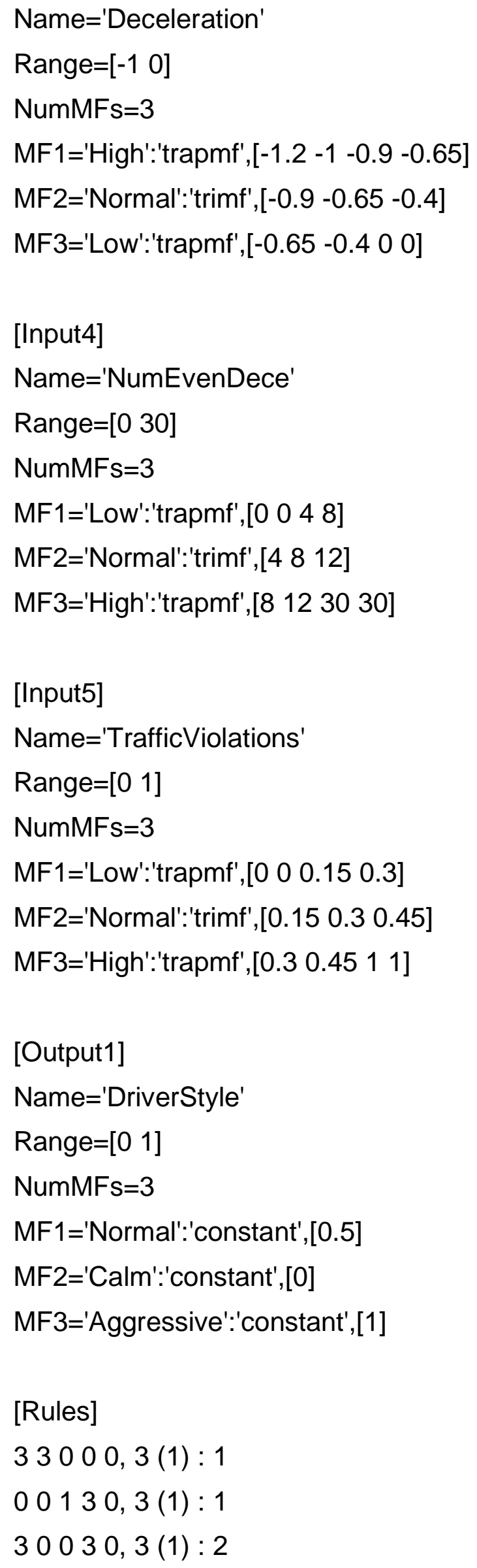

[Rules]

$33000,3(1): 1$

$00130,3(1): 1$

$30030,3(1): 2$ 
$00310,2(0.8): 2$

$02200,1(0.9): 2$

$00003,3(1): 2$

$00001,2(0.8): 2$

$00002,1(0.9): 2$

$20020,1(0.9): 2 s$

Código del modelo de redes neuronales en RStudio

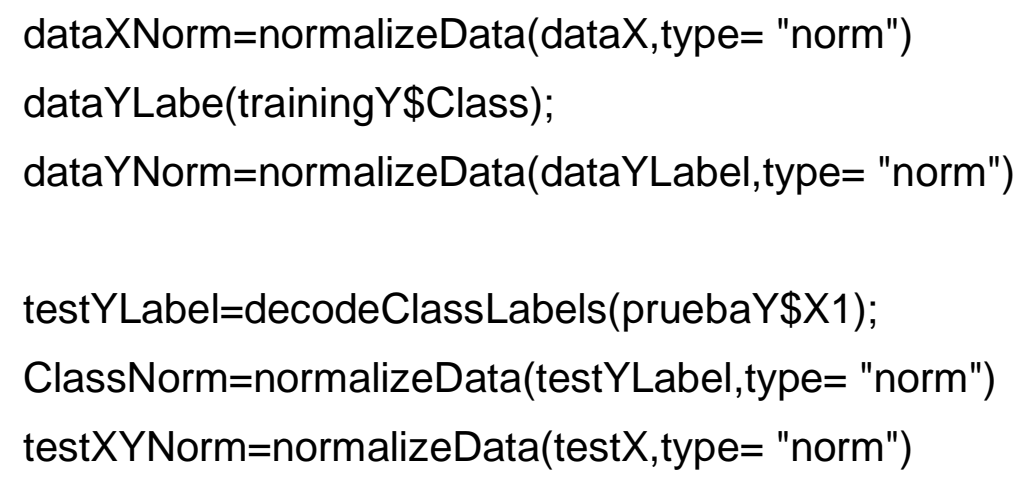

model $=\operatorname{mlp}$ (dataXNorm, dataYNorm, size $=4$, learnFuncParams $=0.4$, learnFunc="BackpropBatch", linOut=TRUE ,maxit=600, shufflePatterns = FALSE)

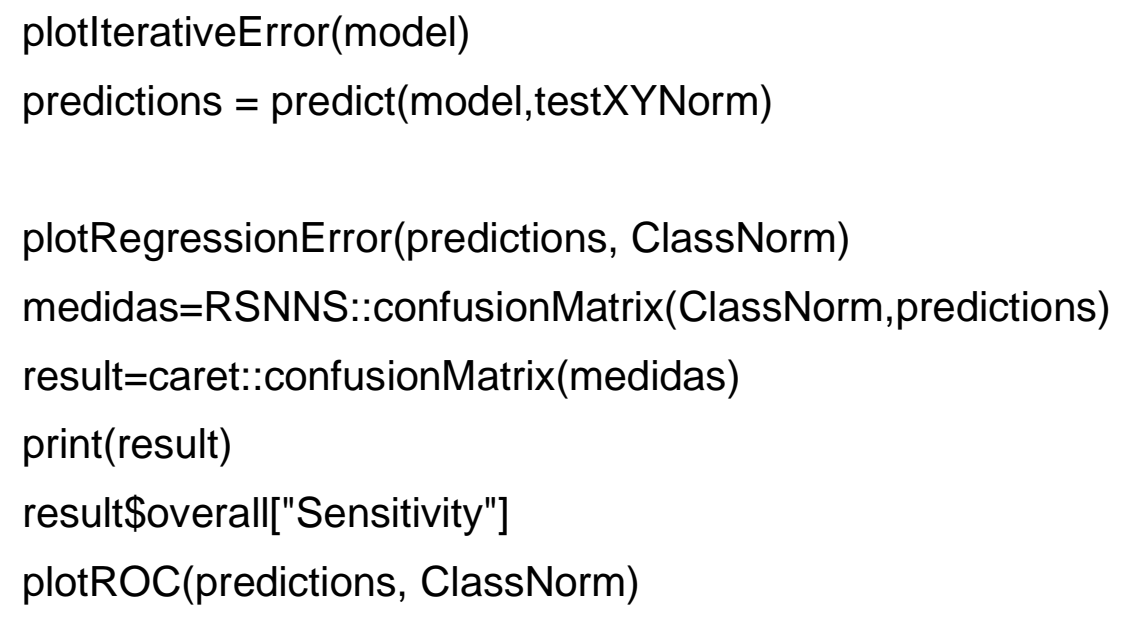

Código del modelo de máquinas de soporte vectorial en RStudio

factorYY=as. factor(trainingCLASS $\$ . .1$ )

factorclass $=$ as.factor $($ LabelClass $\$ . .1)$

for (c in $c(-5: 10))\{$

for $(r$ in $c(-5: 10))\{$

$\cos t=2^{\wedge} \mathrm{C}$ 


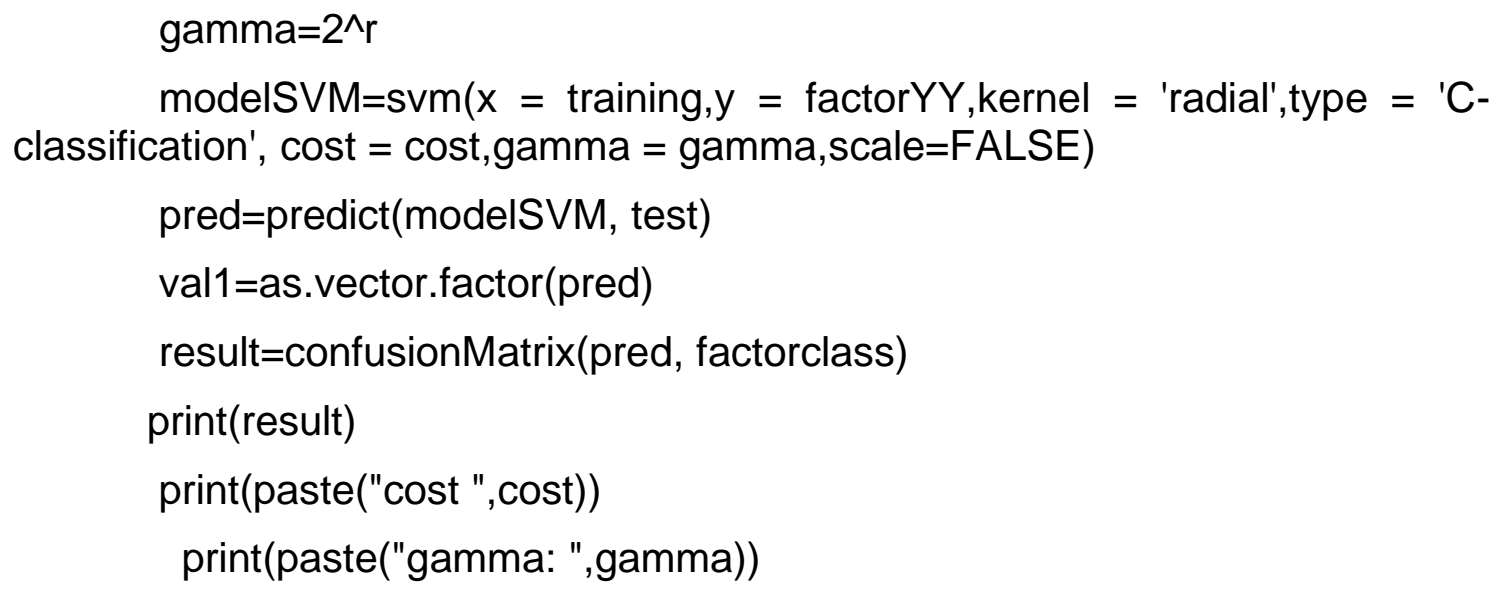

Código del modelo de bosques aleatorios en RStudio

modelRF=randomForest( $\mathrm{x}=$ training, $\mathrm{y}=$ factor(DataSampleFINAL $\$$ Class), xtest=test, ytest=factor(Data $\$$ Class), ntree $=100$, mtry=4)

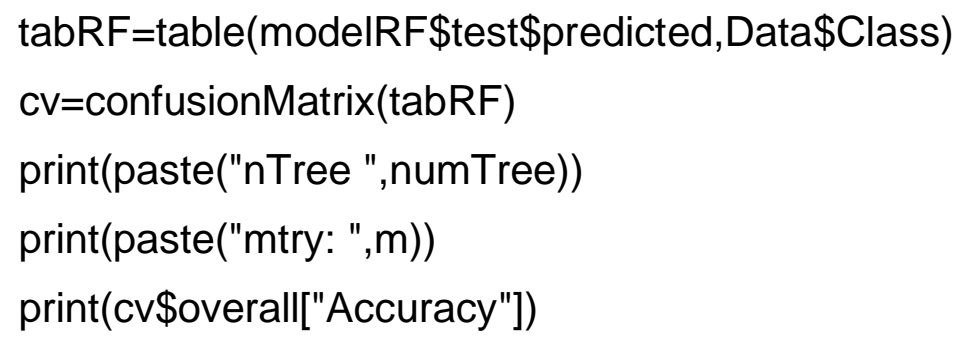

Código del modelo de k-vecinos más cercanos en RStudio

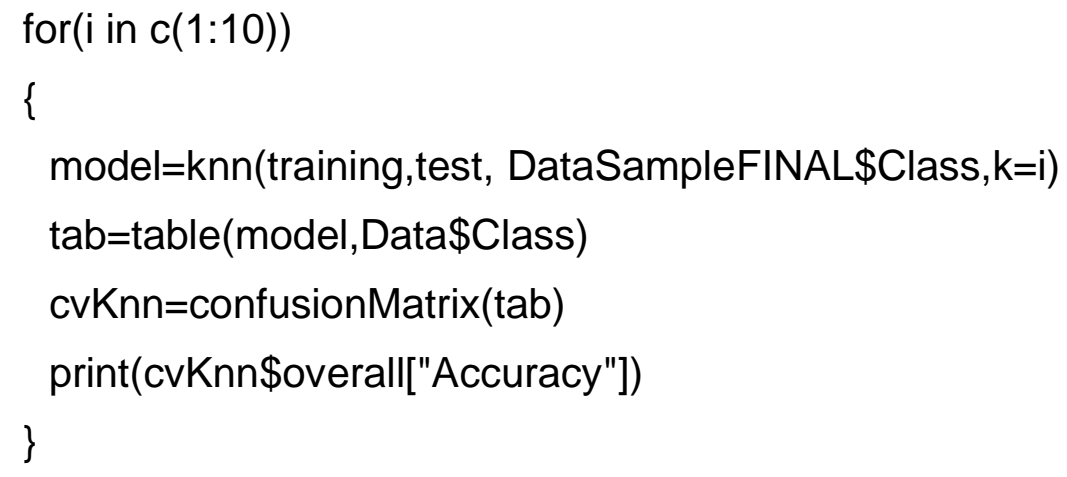

